\&RP SDFW( IIIFHQW/ RZDFRWW / DUHW \& ( / / 3 उ URAFWM LQDO 5 HSRUW

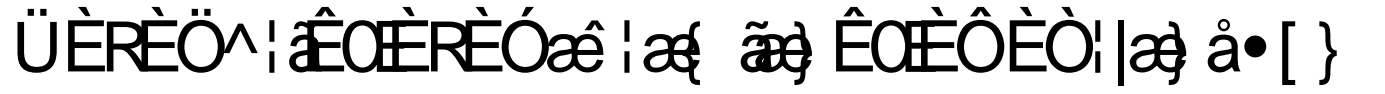

2 FREHUTास००

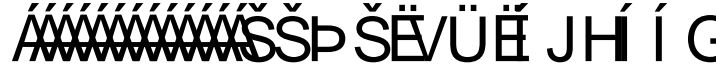


This document was prepared as an account of work sponsored by an agency of the United States government. Neither the United States government nor Lawrence Livermore National Security, LLC, nor any of their employees makes any warranty, expressed or implied, or assumes any legal liability or responsibility for the accuracy, completeness, or usefulness of any information, apparatus, product, or process disclosed, or represents that its use would not infringe privately owned rights. Reference herein to any specific commercial product, process, or service by trade name, trademark, manufacturer, or otherwise does not necessarily constitute or imply its endorsement, recommendation, or favoring by the United States government or Lawrence Livermore National Security, LLC. The views and opinions of authors expressed herein do not necessarily state or reflect those of the United States government or Lawrence Livermore National Security, LLC, and shall not be used for advertising or product endorsement purposes.

This work performed under the auspices of the U.S. Department of Energy by Lawrence Livermore National Laboratory under Contract DE-AC52-07NA27344. 


\title{
Compact, Efficient, Low-cost Lasers (CELL) Project: Final Report
}

Strategic Initiative 10-SI-010

\author{
R. J. Deri, A. J. Bayramian, and A. C. Erlandson \\ October 11, 2012
}

\section{PROJECT MOTIVATION and SCOPE}

Development of clean, sustainable and affordable energy sources is a national and international challenge. Inertial Fusion Energy (IFE) may provide a transformational solution that is scalable, proliferationresistant, and that generates negligible nuclear waste. To produce energy economically competitive with alternative power plants, the IFE laser drive must operate at a high repetition rate $(\geq 10 \mathrm{~Hz})$ with high efficiency (>10\%), while maintaining beam quality suitable for focusing to a small spot suitable for compressing the fusion target. The NIF laser system meets the beam quality requirements using a solidstate gain medium pumped by flashlamps. Scaling from NIF to IFE requires increases of $\sim 10^{5}$ in rep rate and $~ 20 \mathrm{X}$ in efficiency, which adds significant challenges to the laser design. The increased repetition rate results in much more waste heat generated in the system, and the prevention of thermal beam quality degradation (through phenomena such as thermally-induced birefringence) becomes a critical issue. While thermal issues are mitigated by increasing the system volume, a major size increase renders the system less economical and less easy to site. LIFE laser efficiency targets can be achieved at high rep rate without reliability degradation by using diode lasers as pumps. An IFE plant requires $\sim 10^{8}$ diode laser bars. At current costs the diodes dominate the overall laser cost and render IFE less cost-effective. Thus, designing a compact and cost-effective laser system with the repetition rate, efficiency, and beam quality required for IFE presents a significant technical challenge.

The goal of the CELL strategic initiative was to develop new laser architectures to address these challenges by leveraging recent advances in optical technology. New laser design options have become potentially feasible due to improvements in laser gain media, diode pumps, large aperture nonlinear optics, and damage-resistant optics that have occurred since NIF's conception. The project investigated designs that take advantage of these advances, combining them with advanced and novel subsystem concepts to achieve laser designs with significantly improved performance, size, and cost.

\section{APPROACH}

A primary objective of this project was to rapidly evaluate a variety of beamline concepts and architectures, to ascertain their suitability for IFE applications. This is best done in simulation, due to the time and expense associated with prototyping such systems, even at significantly reduced scale. These evaluations were performed rapidly using a laser energetics code ("LPM") specifically developed for this project. This tool tracks all system inefficiencies from wallplug power to the final optic, includes the power consumed by cooling systems, and captures the behavior of different laser materials. In particular, it is suitable for simulating both four-level (e.g.; Nd-based) and three-level (e.g.; Yb-based) gain media. In order to achieve fast run-times, LPM abstracts away details of optical diffraction and propagation, which are represented as scalar losses in various sections of the beamline. The magnitudes of these losses 
were determined from more detailed beamline simulations. New simulation methodologies needed to be developed or added to the simulation codes to handle certain physics and device configurations, including propagation through thermally birefringent materials, behavior of anamorphic spatial filters, and harmonic conversion in devices with non-uniform temperature distributions.

Detailed beamline simulations were performed in four environments:

1. LLNL's PROP92 code, with extensions for non-isothermal frequency conversion, which provides full propagation of single-polarization optical fields, but does not include the details associated with anamorphic filters

2. The CEA's MIRO code, which provides full propagation of arbitrarily polarized fields and anamorphic filters.

3. A custom MATLAB code written for this project (JONESPROP), which enabled rapid assessment of thermal birefringence, depolarization, and nonlinear ellipse rotation, as well as cross-checking the results of the more sophisticated full propagation models.

The propagation models were populated with aberration files taken from actual NIF optics, so that these simulations showed behavior representative of that achievable with non-ideal optics. The thermal, stress, and birefringence inputs to the optical simulations were obtained from thermo-mechanical models. The optical gain distribution and thermal loading in the amplifier heads was obtained from ray tracing simulations of the pump diode delivery system which were performed using customized scripts for the commercial FRED raytracing package. Stress and temperature fields were obtained from LLNL's NIKE and TOPAZ codes, which were cross-checked against commercial ANSYS software, and birefringence was calculated using LLNL's OPL code. Simulation results from these different approaches were generally in close agreement.

The beamline architecture that emerged from this project requires certain new components, notably large scale quartz rotators and grazing-incidence "slits" for anamorphic spatial filters. In order to understand the risks associated with these components, we performed targeted experiments-specifically to assess their robustness to laser-induced optical damage.

\section{PRINCIPAL ACCOMPLISHMENTS}

This project achieved the following key technical accomplishments:

1. Development of a novel laser beamline architecture suitable for IFE, which exhibits $\sim 3 \mathrm{x}$ more efficiency, 30\% less nonlinear phase shift (B-integral), and 2x smaller footprint than the IFE laser design that existed prior to the project. This architecture also reduces the risk of optical damage, by substantially reducing the operating optical fluence on the optics at both $1 \omega$ and $3 \omega$, and the risk of Pockels cell failure by reducing its fluence by $\sim 12 \mathrm{x}$. The basics of this architecture are documented in the publication included as Appendix I of this report. This architecture was adopted as the current baseline for LLNL's IFE effort, and provided the basis for several beamline proposals to external sponsors.

2. Development of a laser performance model ("LPM") that enables rapid assessment of a complete laser beamline, including inefficiencies associated with 3-level laser gain media and cooling subsystems. This tool enables rapid quantification of the tradeoff between laser efficiency and 
cost, and has been adopted by the IFE system development team for use in IFE facility design studies. It is described in detail in the publication included as Appendix II of this report.

3. An assessment of laser cost-efficiency tradeoffs for several gain media alternatives, which quantifies the performance differences between four-level and three-level gain media. This work is described in detail in the publication included as Appendix II of this report.

4. Invention of several new components to support these beamlines, including a thermally-robust harmonic converter with $>70 \%$ tripler conversion efficiency (IL-12360, US Patent application submitted).

5. A detailed assessment of thermal birefringence effects in the proposed beamline, which is detailed in the paper included as Appendix III of this report. This study established the feasibility of the proposed approach for mitigating thermal birefringence, a key risk associated with operating at higher repetition rates.

6. Demonstration of a method for scaling quartz rotators, which is detailed in the paper included as Appendix IV of this report. This work demonstrated the feasibility of fabricating larger quartz rotators by bonding multiple small crystals, addressing potential risks associated with the availability of large optical quartz crystals. This work, and subsequent experiments with larger scale components, showed that the optical behavior of bonded material is suitable for use in the proposed architecture.

7. Demonstration of high damage threshold "slits" for anamorphic cylindrical filters, as described in Appendix V of this report. Use of properly fabricated fused silica in this application yields damage thresholds $>100 \mathrm{~J} / \mathrm{cm}^{2}$, which is suitable for use as a refractive slit in the cylindrical filters proposed for the beamline. These high damage thresholds enable compact filters, which in turn minimize the overall beamline footprint.

The technical details associated with these accomplishments are described in the appendices. 


\section{PUBLICATIONS and INVENTIONS}

The CELL project resulted in several publications and patent applications, which are enumerated below.

\section{Publications and Conference Presentations}

[1] A.J. Bayramian, R.J. Deri, C.A. Ebbers, A.C. Erlandson, W.A. Molander, S.B. Sutton, S. Telford, and J. A. Caird (TUTORIAL), "Fusion Energy: Laser Systems Required to Harness the Power of the Sun”, Conf. Lasers and Electroptics (CLEO, San Jose, CA; May 2010). Paper AThA2

[2] J. A. Caird, A.C. Erlandson, R.J. Beach, A.M. Rubenchik, and C. P. J. Barty, "High Energy Laser Design for Space Debris Removal”, Int. Conf. Orbital Debris Removal (December 2009).

[3] A. J. Bayramian, C. Boley, A. Bullington, D. Chen, R.J. Deri, A. Erlandson, et al. ,’Compact, Efficient Laser Systems Required to Harness the Power of the Sun (INVITED)", ANS $19^{\text {th }}$ Topical Mtg. Technol. Fus. Energy (TOFE, Las Vegas, NV; November 2010). Paper T3-OS-12-1.

[4] A. J. Bayramian, R.J. Deri, S. Fulkerson, R. Lanning, S. Telford, ” Compact, efficient, low-cost diode power conditioning for Laser Inertial Fusion Energy", Proc. SPIE Vol. 7916 (2011), pp. 79160B-179160B-5. Photonics West Conference (San Francisco, CA, January 2011).

[5] A. L. Bullington, S. B. Sutton, A. J. Bayramian, J. A. Caird, R. J. Deri, A. C. Erlandson, M. A. Henesian, "Thermal birefringence and depolarization compensation in glass-based high-average-power laser systems", Proc. SPIE Vol. 7916 (2011), pp. 79160V-1- 79160V-9. Photonics West Conference (San Francisco, CA, January 2011).

[6] A. M. Rubenchik et al., Rubenchik, A. M., et al "Modulation instability in high power amplifiers." Opt. Express 18(2), 1380 (2010). LLNL-JRNL-431613.

[7] A. J. Bayramian, C. Boley, A. Bullington, D. Chen, R.J. Deri, A. Erlandson, et al., “A Compact Line Replaceable Unit Laser Driver for Laser Inertial Fusion Energy”, Conf. Lasers and Electrooptics Paper JTh3I.5 (CLEO, San Jose, CA; 2012).

[8] N. Traggis, N. Claussen, A. Bayramian, K. Schaffers, and R.J. Deri, "Improved characterization of transmitted wavefront error on CADB epoxy-free bonded solid state laser materials”, Proc. SPIE Vol. 7912 (2011), pp. 79121K-1- 79121K-7. Photonics West Conference (San Francisco, CA, January 2011).

[9] K. I. Schaffers, A. Bayramian, A. Bullington, J. Caird, R. Deri, A. Erlandson, M. Henesian, and C. Stolz (INVITED), “Advanced materials for Inertial Fusion Energy Lasers”, 18th Am. Conf. Crystal Growth Epitaxy (Monterey, CA; August 2011). Paper ACCGE-3.

[10] A. J. Bayramian, A. C. Erlanson, et al., "LIFE laser system update". 6 ${ }^{\text {th }}$ High Energy Class Diode Pumped Solid State Lasers Workshop (Versailles, France, September, 2010). LLNL-PRES-452916.

[11] A. J. Bayramian, R W. Campbell, C. A. Ebbers, et al., "A Laser Technology Test Facility for Laser Inertial Fusion Energy (LIFE)”, J. Phys.: Conf. Ser. 244, 032016 (2010)

[12] A. J. Bayramian, A. C. Erlanson, et al., "The Heat capacity laser". $6^{\text {th }}$ High Energy Class Diode Pumped Solid State Lasers Workshop (Versailles, France, September, 2010). LLNL-PRES-452893.

[13] A. Bayramian, S. Aceves, T. Anklam, K. Baker, E. Bliss, C. Boley, A. Bullington, J. Caird, D. Chen, R.J. Deri, M. Dunne, A. Erlandson, D. Flowers, M. Henesian, J. Latkowski, K. Manes, W. Molander, E. Moses, S. Powers, S. Rana, S. Rodriguez, R. Sawicki, K. Schaffers, L. Seppala, M. Spaeth, S. Sutton, S. Telford, "Beam in a box: a compact line replaceable unit for LIFE", $7^{\text {th }}$ Intnl. Conf. Inertial Fus. Sci. Appl. (IFSA, Bordeaux, France; September 2011). Paper O.Mo_B8.

[14] A. Erlandson, T. Anklam, A. Bayramian, R. Beach, C. Boley, A. Bullington, J. Caird, D. Chen, R.J. Deri, M. Dunne, D. Flowers, M. Henesian, K. Manes, W. Molander, E. Moses, S. Rana, K. Schaffers, L. Seppala, M. Spaeth, C. Stolz, S. Sutton, and S. Telford, "Efficiency modeling for Laser Inertial Fusion Energy Drivers", $7^{\text {th }}$ Intnl. Conf. Inertial Fus. Sci. Appl. (IFSA, Bordeaux, France; September 2011). Paper O.Mo_B9.

[15] A.J. Bayramian, “Solid State Lasers: Present and Future”, $2^{\text {nd }}$ Joint ICFA-ICUIL Workshop (2011). 
[16] A. Bayramian, A. Erlandson, R. Deri, et al., "Beam in a Box: A Compact Line Replaceable Unit for Chirped Pulse Amplification”, Ultrafast Optics Conference (UFO-X) (2011).

[17] A. Bayramian, A. Erlandson, R. Deri, et al., "Progress towards a Compact Laser Driver for Laser Inertial Fusion Energy", $7^{\text {th }}$ High Energy Class Diode Pumped Solid State Lasers (HEC-DPSSL) Workshop (September 2012).

[18] A. C. Erlandson, R. J. Deri, A. J. Bayramian, et al., "Comparison of Nd:phosphate glass, Yb:YAG and Yb:S-FAP laser beamlines for laser inertial fusion energy", $7^{\text {th }}$ High Energy Class Diode Pumped Solid State Lasers (HEC-DPSSL) Workshop (September 2012).

[19] A. M. Rubenchik, A. C. Erlandson and D.M. Liedahl, (INVITED) "Laser System for Space Debris Cleaning,” AIP Conf. Proc. High Power Laser Ablation Conference (Santa Fe, New Mexico, 30 April - 3 May, 2012).

[20] M. A. Henesian, A. Bullington, A. Bayramian, et al., "High average power frequency conversion", $7^{\text {th }}$ High Energy Class Diode Pumped Solid State Lasers (HEC-DPSSL) Workshop (September 2012).

[21] A. Bayramian, R.J. Deri, S. Fulkerson, R. Lanning, S. Telford, ”Compact, efficient, low-cost diode power conditioning for Laser Inertial Fusion Energy”, Proc. SPIE Vol. 7916 pp. 79160B-1- 79160B-5. (2011).

[22] A. C. Erlandson, S. M. Aceves, A. J. Bayramian, A. L. Bullington, R. J. Beach, C. D. Boley, J. A. Caird, R. J. Deri, A. M. Dunne, D. L. Flowers, M. A. Henesian, K. R. Manes, E. I. Moses, S. I. Rana, K. I. Schaffers, M. L. Spaeth, C. J. Stolz, and S. J. Telford (INVITED), “Comparison of Nd:phosphate glass, Yb:YAG and Yb:S-FAP laser beamlines for laser inertial fusion energy (LIFE)”, Opt. Mat. Express 1, pp. 1341-1352 (2011). LLNL-JRNL-486773

[23] A.C. Erlandson, A.J. Bayramian, et al. (INVITED, “diode-pumped Nd:glass laser beamlines for Laser Inertial Fusion Energy (LIFE) (invited)”, ANS 20 ${ }^{\text {th }}$ Topical Mtg. Technol. Fus. Energy (TOFE, Nashville, TN; August 2012). Paper T3-OS-7-2.

[24] A.J. Bayramian, S. Aceves, T. Anklam, et al., "Compact Efficient Laser Systems Required for Fusion Energy”, Fus. Sci. Technol. 60, 28 (2011). LLNL-JRNL-462671.

Inventions (filing dates refer to USPTO filings)

[1] R.J. Deri, J. Kotovsky, and C.M. Spadaccini, “Laser Diode Package with Enhanced Cooling”, US Patent No. 8,018,980 (IL-12126, September 13, 2011)

[2] R.J. Deri, D. Chen, A. Bayramian, B. Freitas, and J. Kotovsky, "Enhanced Vbasis Laser Diode Package”, US Application 20110235669 (IL-12123, March 2010)

[3] R.J. Deri, A.C. Erlandson, A.J. Bayramian, and R.J. Beach, "Cryocooled Laser Amplifier with Warm Emission Absorber” (IL-12308, filed January 2011).

[4] R.J. Deri, M.A. Rhodes, A.J. Bayramian, J.A. Caird, M.A. Henesian, and C. Ebbers, "Electro-optic Device with Gap Coupled Electrode”, US Patent Application No. 2012105931 (IL-12306, filed July 2010).

[5] A.J. Bayramian, K.R. Manes, R.J. Deri, A.C. Erlandson, , J.A. Caird, and M.L. Spaeth, "Transverse Pumped Laser Architecture” (IL-12303, filed October 2010)

[6] A.J. Bayramian, J. Latkowski, R. Sawicki, and R.J. Deri, “Final Beam Transport System” (IL-12374, filed January 2011)

[7] J.A. Caird and K.I. Schaffers, "Method and System for Mitigation of Inclusions in Optical Materials by Laser Scanning” (IL-12291, filed December 2011)

[8] A.C. Erlandson, J.A. Caird, M.A. Henesian, K.R. Manes, R.J. Deri, A.J. Bayramian, and M.L. Spaeth, "Pulse Reformatting for Efficiency and Redundancy" (IL-12359, filed November 2010)

[9] J.A. Caird, ““'Suppression of Simulated Raman Scattering (SRS)” (IL-12302, filed October 2011)

[10] A.J. Bayramian, A.C. Erlandson, K.R. Manes, M.L. Spaeth, J.A. Caird, and R.J. Deri, "Compact Efficient Laser Architecture”, US Patent Application No. 2012105948 (IL-12351, filed November 2010) 
[11] J.A. Caird and K.S. Schaffers, "Mitigation of metallic inclusions in optical materials by laser scanning at high temperature” (IL-12291, filed January 2011)

[12] J.S. Farmer, A. Rubenchik, B.S. El-Dasher, and R.J. Deri, "Use of Diode Arrays for High-Efficiency LightAssisted Friction Stir Processing and Welding of Metals \& Metallic Alloys - Possible Application to Ceramics \& Plastics”, (IL-12527, filed December 2011).

[13] M.A. Henesian, A.L. Bullington, K.R. Manes, M. Spaeth, R. Deri, and C.A. Ebbers "Frequency conversion system of optical system, has frequency doubler module which is arranged along beam path”, Patent No. WO2012082262-A1 (IL-12360, filed January 2011).

[14] A.C. Erlandson, "Spatial Filters for High Average Power Lasers,” US Patent Application \# 20110043899, (IL 12336, filed February 2011).

[15] A.C. Erlandson, "Method and System for Compact and Efficient High Energy Pulsed Laser”, (IL-12169a, filed September 2010)

[16] A.C. Erlandson, "Method and System for Compact, Multi-Passed Pulsed Laser Amplifier”, (IL-12169b, filed September 2010)

[17] K.R. Manes, M. Spaeth, and A. C. Erlandson, "Multi-pass Amplifier Architecture for High Power Laser Systems”, (IL-12246, filed March 2011)

[18] R.J. Deri, T.M. Anklam, R.R. Miles, et al., "Inertial Confinement Fusion Power Plant Which Decouples Life-Limited Components From Plant Availability” (IL-12358, filed November 2011)

[19] A.J. Bayramian, "Method and System for Modulation of Gain Suppression in High Average Power”, US Patent No. 8233511 (IL-12183, granted July 2012)

[20] A.J. Bayramian, "Optical gain architecture for optical systems e.g. laser amplifier system, has cladding sections that are proximate to two of four sides of gain region respectively", US Patent Application No. 2012114008 (IL-11836). 


\section{APPENDICES}

I. A.J. Bayramian et al., "Compact Efficient Laser Systems Required for Laser Inertial Fusion Energy”, LLNL-JRNL-462671.

II. A.C. Erlandson et al., "Comparison of Nd:phosphate glass, Yb:YAG and Yb:S-FAP laser beamlines for laser inertial fusion energy (LIFE)”, LLNL-JRNL-486773.

III. A.L. Bullington et al., "Thermal birefringence and depolarization compensation in glass-based highaverage-power laser systems”, LLNL-ABS-442912.

IV. N. Traggis et al., "Improved characterization of transmitted wavefront error on CADB epoxy-free bonded solid state laser materials”, LLNL-PROC-464931.

A.J. Bayramian et al., “Large, Bonded Quartz Rotators with High Damage Threshold” (unpublished).

V. A.J. Bayramian et al, "Spatial filtering for IFE Lasers” (unpublished). 


\title{
COMPACT, EFFICIENT LASER SYSTEMS REQUIRED FOR LASER INERTIAL FUSION ENERGY
}

\author{
A. Bayramian, S. Aceves, T. Anklam, K. Baker, E. Bliss, C. Boley, A. Bullington, J. Caird, D. Chen, R. Deri, M. Dunne, A. \\ Erlandson, D. Flowers, M. Henesian, J. Latkowski, K. Manes, W. Molander, E. Moses, T. Piggott, S. Powers, S. Rana, S. \\ Rodriguez, R. Sawicki, K. Schaffers, L. Seppala, M. Spaeth, S. Sutton, S. Telford
}

Lawrence Livermore National Laboratory, 7000 East Ave., L-470, Livermore, CA 94551, email: bayramian1@llnl.gov

This paper presents our conceptual design for laser drivers used in Laser Inertial Fusion Energy (LIFE) power plants. Although we have used only modest extensions of existing laser technology to ensure nearterm feasibility, predicted performance meets or exceeds plant requirements: $2.2 \mathrm{MJ}$ pulse energy produced by 384 beamlines at $16 \mathrm{~Hz}$, with $18 \%$ wall-plug efficiency. High reliability and maintainability are achieved by mounting components in compact line-replaceable units that can be removed and replaced rapidly while other beamlines continue to operate, at up to $\sim 13 \%$ above normal energy, to compensate for neighboring beamlines that have failed. Statistical modeling predicts that laser-system availability can be greater than $99 \%$ provided that components meet reasonable mean-time-between-failure specifications.

\section{INTRODUCTION}

Experiments on inertial-confinement fusion (ICF) targets are now underway at the National Ignition Facility $(\mathrm{NIF})^{1,2}$ at Lawrence Livermore National Laboratory (LLNL), and are anticipated to demonstrate fusion ignition within the next few years. The LIFE concept proposes to exploit this scientific breakthrough for energy generation, as described elsewhere., ${ }^{3,5}$ This approach minimizes target-performance risks by using target designs that can be tested on NIF and by using laser parameters and target-illumination geometries similar to NIF.

Another important feature of the LIFE design is the use of harmonically-converted, Nd:glass laser beamlines, which have great similarity to the NIF beamlines. This choice enables the reuse of much of the NIF technology and manufacturing base for LIFE. The LIFE laser design differs from NIF in several respects, however. While the NIF laser slabs are pumped by flashlamps, the LIFE laser slabs are pumped by laser diodes. Diodes pump laser slabs more efficiently than flashlamps, due to their high degree of directionality and narrow spectral emission. Additionally, diodes are more reliable, have longer lifetimes, and can produce more intense, useful pump light. This last property is important for producing higher gain coefficients and stored-energy densities, which in turn enables a high efficiency. Another difference is that while NIF laser slabs are passively cooled, the LIFE laser slabs are actively cooled, by flowing helium gas at high velocity in narrow channels between laser slabs. Active cooling enables operation at high repetition rate, by removing waste heat produced by slab pumping processes.

Because of its use of diode pumping and gas cooling, the LIFE laser has much in common with the Mercury laser system, a $10 \mathrm{~Hz}, 60-\mathrm{J}$, diode-pumped laser at LLNL that also uses flowing gas to cool the laser slabs. Mercury operated for more than 300,000 shots with slab surface heat flux $\left(\sim 1 \mathrm{~W} / \mathrm{cm}^{2}\right.$ per surface $)$, amplifier slab thermal stresses relative to yield stress $(\sim 20 \%)$, and Pockels Cell fluence all similar to the LIFE point design, Thus, Mercury provides a subscale demonstration of the key technologies employed to enhance the NIF laser for LIFE applications. $^{6}$

A LIFE power generation system will have to meet all of the basic requirements of the NIF including laser requirements, target geometry, target illumination, hazardous materials handling and radiation safety. In addition, the power generation system will have to address high average power operation of the laser system, average power effects on the target chamber, target injection and tracking, target mass production, blanket and tritium production, and the balance of plant to produce electricity. A typical power plant must have an expected lifetime of $>60$ years, which at a $16 \mathrm{~Hz}$ repetition rate would require more than 30 billion shots and 30 billion targets.

A LIFE power plant must be commercially attractive; the cost of electricity (COE) and required capital investment in the plant must be minimized, and LIFE must be competitive with other forms of energy production. The other requirement for commercial power production is availability. Toward this end the Reliability, Availability, Maintainability, and Inspectability (RAMI) of the laser beamline must be optimized. Both the financial constraints imposed by the COE and operational constraints imposed by RAMI impose a heavy burden on 
the laser. Computer optimization codes are utilized to analyze this multivariable problem. Examination of laser subsystems provides insight into how technological and materials developments can provide the pathway for success in construction of LIFE systems. The LIFE subsystems include: the laser architecture, diode laser pump source, diode light delivery, optics and laser gain media, cooling systems, gain isolation, beam control, frequency converters, and final beam transport optics.

Several new technologies and architectures have been proposed to address these issues based on the successful deployment of NIF (essentially the full scale laser energy required for a LIFE plant), the Mercury laser system ${ }^{6}$ (a sub-scale diode pumped solid state laser), and recent technological advances. These advancements yield wallplug efficiencies of $10-20 \%$, decreased size, and lower cost per Joule. The models and enhancements to the baseline LIFE design (Fig. 1) will be discussed along with realization of a LIFE demo plant.

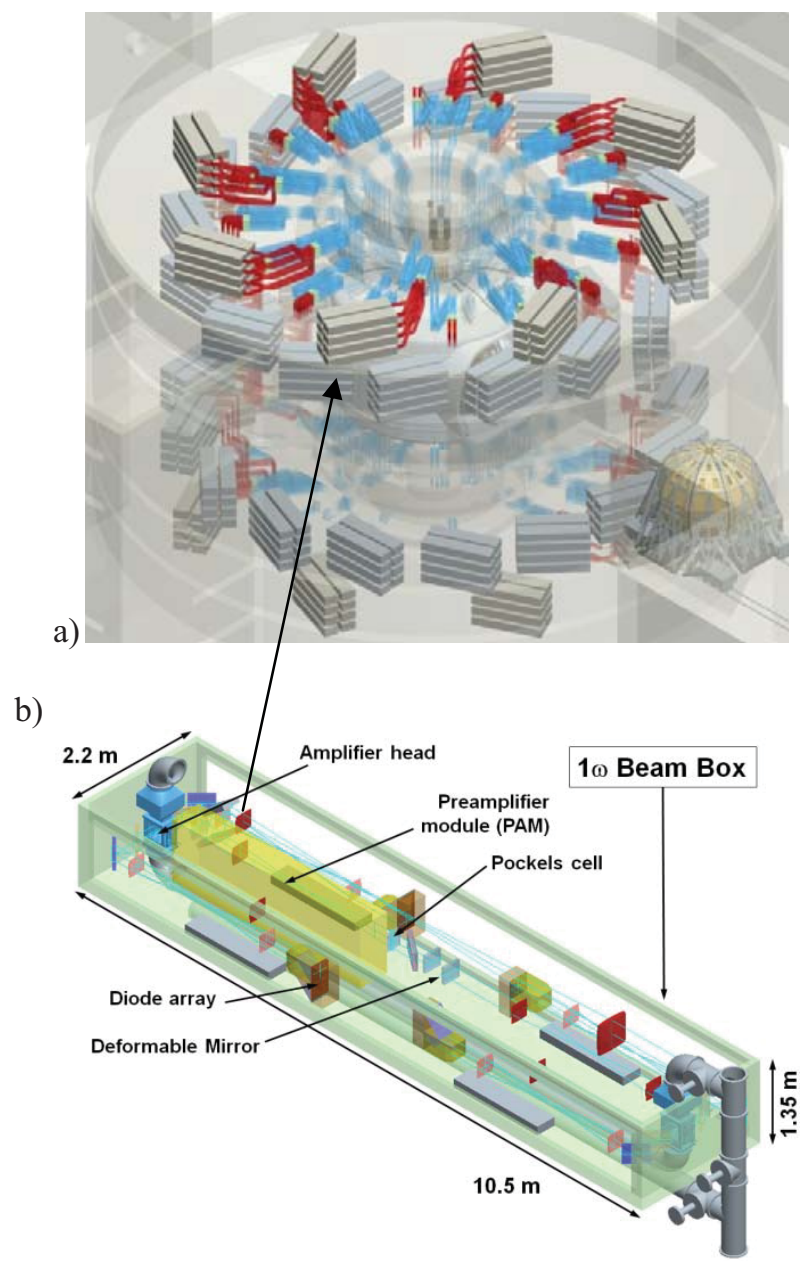

Fig. 1 a) Isometric view of a LIFE power plant showing compact beam architecture, b) Isometric view of an expanded view showing the contents of a beam box.

\section{SYSTEM REQUIREMENTS}

The essential goals of this new beamline design for LIFE are best summarized by RAMI (Reliability Availability, Maintainability, and Inspectability) as the guiding principles. While it might appear that one must sacrifice performance to achieve these goals, the overall efficiency and cost of electricity were actually improved by incorporating RAMI considerations into our design process. To quantitatively assess the impacts of our design decisions on the availability and maintainability of a LIFE plant, Monte Carlo simulations of the Line Replaceable Unit (LRU) components of a beamline were used along with educated estimates of the Mean Time To Failure (MTTF) of individual subcomponents. MTTF is used to refer to components which wear out and are discarded or recycled (such as capacitors, o-ring seals, etc.) While our overall goal for the beamline LRU's is to get MTTFs that are commensurate with the plant lifetime since this would also mitigate maintenance costs, an availability of $>99 \%$ is possible with a Mean Time Between Failure (MTBF) of only a few thousand hours (Fig. 2). MTBF is used to refer to a component or LRU which requires maintenance. Critical to these estimates is the Mean Time To Repair (MTTR), which is shown by these models to require 8 hours or less to achieve $99 \%$ availability. MTTR is used to refer to the replacement time of an LRU. A short maintenance time mandates the LRU concepts we have already adopted for the laser architecture which rely on small footprint, low weight, and kinematic mounting for transportability and replacement.

Table I gives the top level laser system requirements based on the RAMI principles, LIFE power requirements, and the beamline ignition requirements derived from NIF. For more detailed information on LIFE plant operation including circulating power, gain, cost of electricity, and capital cost, please see concurrent papers. ${ }^{3,5}$ Notable here is the wallplug efficiency and repetition rate which are necessary for efficient power production. The large number of beamlines enables the plant availability while maintaining proper target illumination. It should be noted that the lifetime of the system, $30 \times 10^{9}$ shots corresponds a $\sim 60$ year lifetime of the system at $16 \mathrm{~Hz}$, NOT the Mean Time Between Failure (MTBF). While the laser system will be built with this lifetime as a requirement, the complexity of the laser system combined with statistical failure of subcomponents and/or servicing needs will necessarily result in a MTBF less than the lifetime. A survey of commercial pulsed high energy diode pumped solid state laser systems indicates they are commonly guaranteed for 10,000 hours, which by necessity would be less than MTBF. While the fluence loading of optics in these systems are not the same, these systems share a commonality with LIFE laser beamlines in their need for coolant systems, fluid and gas seals, power connectors, 
diode power conditioning, diode arrays, control electronics, stability control, alignment control, and average power handling. In Mercury, where the fluence was commensurate with LIFE operational levels, it was all of the aforementioned issues which dominated our operational MTBF. From the analysis shown in Fig. 2, it would appear a MTBF of 2000 hours would be sufficient even for an $8 \mathrm{hr}$ maintenance cycle, but the authors consider this number to be the lower bound for LIFE systems. Clearly a larger MTBF will lower maintenance costs and ease operation of the system.

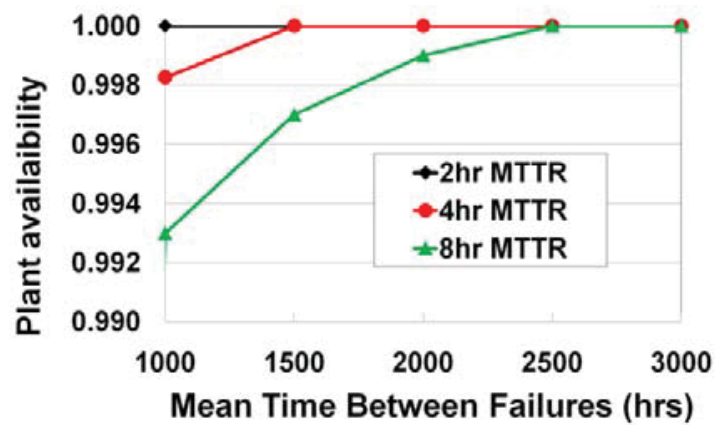

Fig. 2 Plant availability as a function of the Mean Time Between Failure (MTBF) showing potential for high availability with modest MTBF and MTTR

TABLE I. Top level laser system requirements

\begin{tabular}{|c|c|}
\hline Characteristic & Requirement \\
\hline Total laser energy & $2.2 \mathrm{MJ}$ \\
\hline Total peak power & 633 TW \\
\hline \# beamlines & $384(48 \times 8)$ \\
\hline Energy per beamline $(3 \mathrm{w})$ & $5.7 \mathrm{~kJ}$ \\
\hline Wallplug efficiency & $15 \%$ \\
\hline Repetition rate & $16 \mathrm{~Hz}$ \\
\hline Lifetime of system & $30 \times 10^{9}$ shots \\
\hline Availability & 0.99 \\
\hline Maintenance & $<8 \mathrm{hrs}$ \\
\hline Beam pointing & $100 \mu \mathrm{m} \mathrm{rms}$ \\
\hline $\begin{array}{l}\text { Beam group energy stability } \\
\text { ( } 8 \text { beams) }\end{array}$ & $<4 \% \mathrm{rms}$ \\
\hline Beam to beam timing at target & $<30$ ps rms \\
\hline Focal spot (w/ CPP*), 95\% enclose & $3.1 \mathrm{~mm}$ \\
\hline Spectral bandwidth, $3 \omega(\mathrm{GHz})^{* *}$ & 180 \\
\hline Prepulse (20 ns prior to main) & $<10^{8} \mathrm{~W} / \mathrm{cm}^{2}$ \\
\hline
\end{tabular}

* CPP = Continuous Phase Plate - used to modify the far field from a peak to a flat top for target drive

** Used for suppression of Stimulated Raman Scattering, Stimulated Brillouin Scattering, and in conjunction with a diffraction grating for Smoothing by Spectral Dispersion (SSD) of the laser speckle induced by use of the CPP on target.

\section{LASER ARCHITECTURE}

To meet the reliability, availability, and maintainability requirements for the laser system, the whole architecture is designed to be packaged into Line Replaceable Units (LRUs) which are assembled, tested, and serviced at a factory and transported to the LIFE plant via standard truck. The LRUs for the beamline include: a $1 \omega$ beam box which houses the entire $1 \omega$ beamline, a frequency converter, transport mirrors, and final optics. The $1 \omega$ beamline is packaged in a box with rough dimensions 2.2 meters wide $\mathrm{x} 1.4$ meters tall $\mathrm{x} 10.5$ meters long (Fig. 1b). This box requires only an electrical connection, communications lines, water cooling lines, and helium gas cooling lines.

The 2.2 MJ required for robust, high gain ignition at the target chamber center ${ }^{7}$ is provided by 384 beamlines (48 clusters of 8 beams), which each produce $8.1 \mathrm{~kJ}$ at $1053 \mathrm{~nm}$. This energy is frequency converted and transported to target providing $5.7 \mathrm{~kJ}$ at $3 \omega$ on target.

The availability of the power plant is maintained at a high level by allowing the $1 \mathrm{w}$ beam-box units to be replaced while the plant continues to run. This is achieved through the introduction of a large number of beamlines, each of which can operate at a higher level than nominal to compensate for a nearby non-operational unit. This design philosophy allows a short MTBF to be tolerated while maintaining plant operations.

\section{III.A. Optical Architecture}

The LIFE design (Fig. 3) can be understood in greater detail by following the laser pulse through the system. A $\sim \mathrm{nJ}$ pulse produced in the master oscillator room (MOR) is transported by optical fiber to each $1 \omega$ beam box. In a preamplifier module (PAM) similar to the one used on NIF, an amplitude modulator shapes the pulse temporally, a regenerative amplifier amplifies the beam, a beam shaper tailors the regenerative amplifier output spatially, and a four-pass amplifier increases pulse energy to $\sim 0.5-1 \mathrm{~J}$. The PAM for LIFE will have $10 \mathrm{X}$ lower energy requirement $(\sim 1 \mathrm{~J})$, but at higher repetition rate $(16 \mathrm{~Hz})$, which is readily achievable with diode pumped Nd:YLF amplifiers (having the same laser wavelength as the Nd:glass gain medium). It should be noted here that the spectral bandwidth requirement in Table 1 is achieve in an identical manner to the method used on NIF, through the use of integrated RF modulators in the fiber optic portion of the front end. The seed pulse is then injected into the main LIFE laser amplifiers, which employ a 4-pass image-relayed architecture similar to NIF. The PAM output is injected into the main $1 \omega$ amplifier beamline using a mirror located near the far field of the expansion telescope (fig. 3), which expands and collimates the beam to a $\sim 25 \mathrm{~cm} \times 25 \mathrm{~cm} 1 \omega$ beam 
size. The beam is then reflected by a $1 \omega$ mirror and a polarizer into the amplifier cavity. The beam enters the cavity s-polarized with respect to the polarizer and is converted to circular polarization by a quarter waveplate. Circular polarization lowers the nonlinear phase shift by $\sim 33 \%$ relative to linearly polarized light. The beam then passes through the first amplifier, the spatial filter, the quartz rotator, and the second amplifier. Provided the two amplifiers are pumped and constructed similarly to achieve similar thermal birefringence distributions, the $90^{\circ}$ polarization rotation imparted by the quartz rotator causes the thermal birefringence of one amplifier to cancel the thermal birefringence of the other. The beam propagates through a telescope, is reflected by an adaptive optic at the end of the cavity, and passes back through the chain. As reflection changes the handedness of the circularly-polarized light, the beam exits the quarter-wave plate p-polarized with respect to the cavity polarizer. The beam passes through the polarizer, through the Pockelscell, and is incident on a second polarizer that is oriented a 90 degrees with respect to the first. Provided high voltage is applied to its electrodes, the Pockels cell rotates beam polarization by 90 degrees, allowing the beam to pass through a second polarizer (not shown in Fig. 1). After the beam is reflected back through the chain by the second cavity mirror, the beam makes two more passes through the amplifiers. On the last pass, the quarter-wave plate rotates the beam to the s-polarization state relative to the main polarizer and the beam reflected out of the cavity. The various telescopes and spatial filters in the beamline relay the image of an apodized aperture in the PAM to the harmonic converters and to the final optic.

The expansion telescope increases beam dimensions to $41.7 \mathrm{~cm} \times 41.7 \mathrm{~cm}$ and relays the image plane to the frequency converter. The beam then passes through three final transport telescopes, which utilize two "neutron pinholes" to protect the laser system from neutrons generated in the LIFE chamber. The second transport telescope applies an additional magnification factor to the beam, which reaches dimensions of $\sim 48.6 \mathrm{~cm} \mathrm{x}-48.6 \mathrm{~cm}$. Propagation modeling shows that magnification to this size ensures that the $\mathrm{f} \#$ (focal length divided by beam width) of the final optic is sufficiently small to achieve the desired focal-spot size at the target (Table I). The final telescope incorporates the final optic (details section XII), a Fresnel lens which remains robust against target generated neutrons, which deflects the laser beam to the target to eliminate line of site for the same neutrons through neutron pinhole 2 .

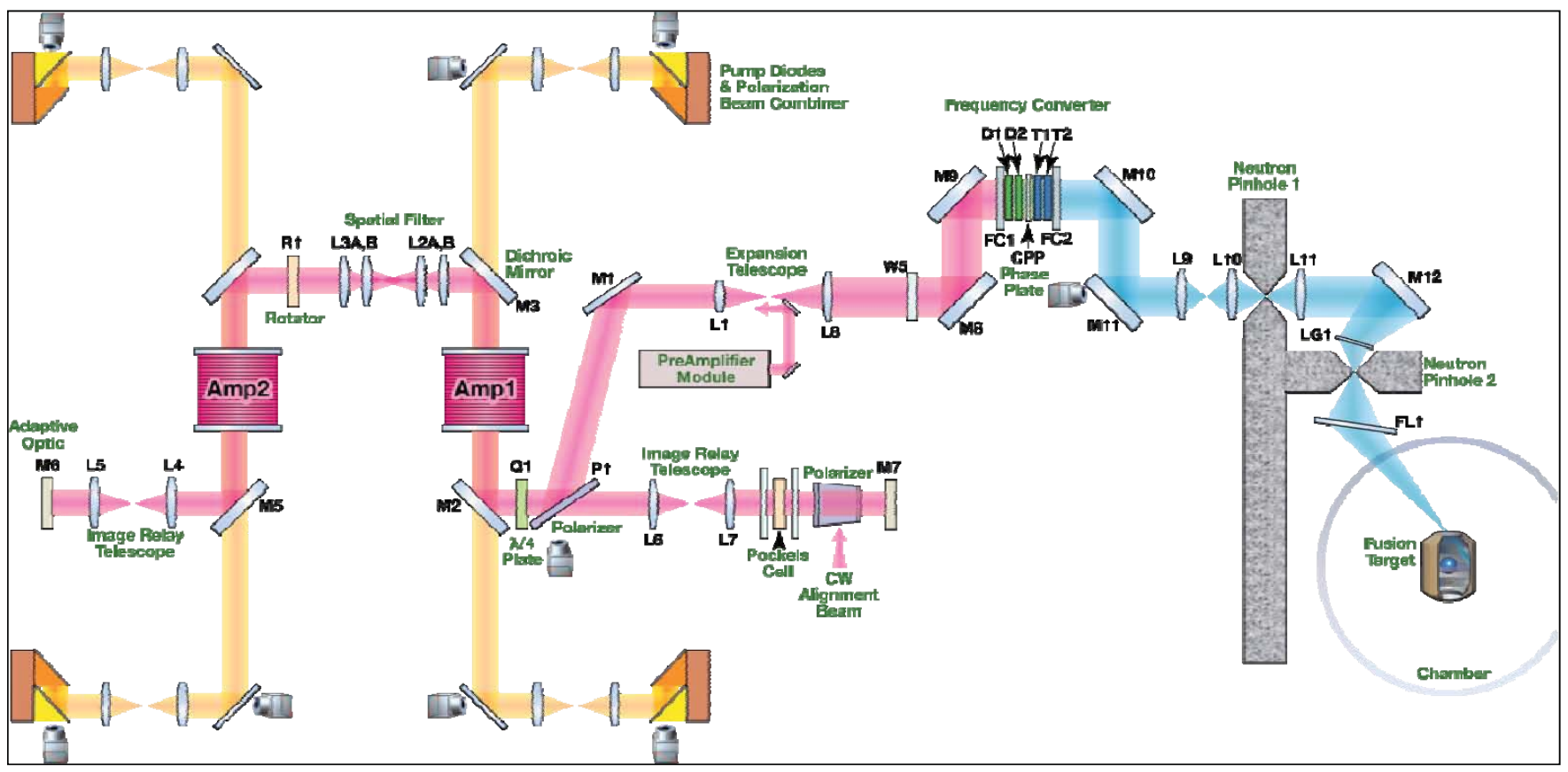

Fig. 3 LIFE laser architecture

\section{III.B. Diode Pumping}

The architecture for the LIFE laser system builds upon the NIF and Mercury designs. To achieve efficient operation, the flashlamps utilized on NIF are replaced with laser diode pumped amplifiers. Raw AC power is rectified to produce continuous DC power at low current, which is stored in capacitors, and switched out using compact and efficient pulser circuits to form high current (700 Amp) electrical pulses which are $\sim 164 \mu$ s long. The 
pulsers are connected to 50-bar stacks of laser diodes each capable of $850 \mathrm{~W}$ peak power for a total of $42.5 \mathrm{~kW}$ per stack. Approximately 770 of these stacks are mounted in a regular grid upon a copper cooling plate to make an array with a peak power of $33 \mathrm{MW}$. The electrical pulsers are cooled on the back side of the same copper plate (see full description of diodes in section 4). Two 33 MW arrays pump each amplifier head. The output from each diode array is relay imaged onto the amplifiers to provide a uniform pump profile for maximum extraction efficiency (see section 5 on the pump delivery). The amplifier heads are an assembly of twenty high speed helium gas cooled amplifier slabs composed of Nd:glass, whose doping concentration is designed to absorb $99 \%$ of the pump radiation (section VI). The wavelength of the diodes, 872 $\mathrm{nm}$, was chosen to minimize the quantum defect which maximizes the wallplug efficiency and minimizes the thermal load to the amplifier slabs.

\section{III.C. Cooling}

The $16 \mathrm{~Hz}$ repetition rate is achieved using the same methodology used on the Mercury laser, namely high speed helium gas cooling. ${ }^{8,9}$ The turbulent helium flow efficiently removes the heat from the surface of the normal incidence laser slabs. This longitudinal heat extraction geometry enables high average power operation without the large self focusing problems observed in radially cooled architectures. Helium gas is chosen to minimize scattering from the gas which is proportional to the square of the change in the refractive index. The Gladstone-Dale coefficient relates refractive index change $(\Delta \mathrm{n})$ to relative changes in gas density $(\Delta \rho / \rho): \Delta \mathrm{n}=\mathrm{G} \Delta \rho / \rho$, where density changes are related to temperature by the ideal gas law $(\rho=\mathrm{p} / \mathrm{RT})$. Helium's Gladstone-Dale coefficient $\left(0.36 \times 10^{4}\right)$ is $7.4 \mathrm{X}$ lower than nitrogen or air, which minimizes the scattering and which led to no observable phase distortion on the Mercury laser system (see section VIII for detail on the cooling system).

\section{III.D. Spatial filtering}

A common technique used to improve beam quality in high energy systems is the use of a small aperture (pinhole) in a relay telescope as a spatial filter. This limits the gain of high spatial frequency near field amplitude modulation due to accumulation of nonlinear phase distortion, and holds off beamline parasitics. At high average power the grazing incidence cone pinholes typically used for this purpose exhibit plasma blow-off on each shot, since the fluence levels are significantly above the damage threshold of the material (typically steel, tungsten, or diamond pinhole). ${ }^{10,11}$ To alleviate this problem, LIFE will use a pair of longitudinally displaced, orthogonal cylindrical relay telescopes (Fig. 4). The longitudinal displacement of the $1 \mathrm{X}$ relay telescopes does not affect the object or relay image points in either axis, but does separate the foci in each axis thereby creating line-foci where this intensity is lower by $\sim 100 \mathrm{X}$. Filtering can then be accomplished with slits instead of pinholes at fluences engineered to be below the damage threshold of the slit material. Since the slits are at the far field in one axis, the net spatial filtering response vs. far field angle is the same as a square cone pinhole (which is ideally matched to the large square beams used on NIF, Mercury, and LIFE). Note that while fig. 4 shows a single on-axis pinhole for clarity, a multiplexed version of this telescope can be realized by replacing each on-axis slit with two off-axis slits. Ablation and damage of the slit material can be avoided by employing a slit material such as fused silica at grazing incidence, which has a very high damage threshold $\left(\sim 475 \mathrm{GW} / \mathrm{cm}^{2}\right) .{ }^{12}$ This high threshold is due to the fact that most of the light is reflected at grazing incidence, and the light that is transmitted is only refracted and not absorbed by the fused silica. Our baseline design is to load the slit at only a fraction of this intensity, $\sim 10 \mathrm{GW} / \mathrm{cm} 2$. A complete $1 / 3$ scale telescope is being fielded tested in 2011 to validate this concept.

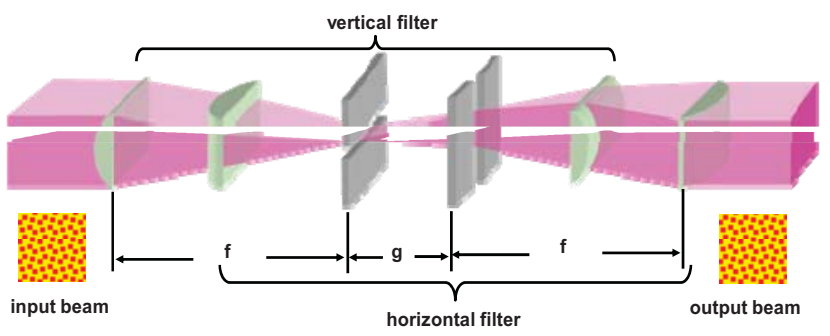

Fig. 4 Cylindrical spatial filter concept showing laser line focus thereby lowering the laser intensity on the filters enabling long lifetime

\section{III.E. Depolarization}

While helium gas cooling enables high average power operation with a tractable induced wavefront, the induced thermal gradient in the amplifier slabs causes thermally induced stress birefringence. A detailed thermal model methodology was used to calculate this effect (Fig. $5){ }^{13}$ Diode pump radiation is ray traced through the amplifier head to produce a thermal profile. The thermal deposition is converted by a finite element model into a stress field. Ray tracing of laser light through the modified optical slab produces a depolarization map. Multi-slab effects in an amplifier can then be evaluated using cascaded Jones matrix analysis. 


\begin{tabular}{|c|c|c|c|}
\hline $\begin{array}{c}\text { Heat } \\
\text { deposition } \\
\text { data }\end{array}$ & $\begin{array}{l}\text { Finite- } \\
\text { element } \\
\text { thermal } \\
\text { and stress } \\
\text { analysis }\end{array}$ & $\begin{array}{c}\text { Ray-trace } \\
\text { analysis of } \\
\text { depolarizat } \\
\text { ion for a } \\
\text { single slab }\end{array}$ & $\begin{array}{c}\text { Multi-slab } \\
\text { Jones } \\
\text { matrix } \\
\text { analysis }\end{array}$ \\
\hline
\end{tabular}

Fig. 5. A flowchart summarizes the numerical analysis of the thermal birefringence.

Based on these calculations, the induced average depolarization in a single slab is $6 \%$, while the combined average depolarization of an amplifier head is $\sim 20 \%$. If the depolarization is not corrected, this light will be lost after transmission through the cavity polarizer. The quartz rotator in Fig. 3 rotates the polarization $90^{\circ}$, which identically cancels these depolarization effects if the optical conditions are identical in each amplifier. ${ }^{14,15}$ The amplifiers are pumped identically and 1:1 image-relayed, which meets this requirement to first order. Second order effects detract from perfect compensation including: magnification between amplifiers, differing thermal loads, differing alignment of the extraction beam on the pump profile, and differing B-integral values present during the extraction pass. Using these same codes, a sensitivity analysis of these secondary effects was performed. The results indicate that with careful engineering of the system, the combined effect of these errors can be limited to $<1 \%$ loss through the entire $1 \omega$ beamline (see Fig. 6 , with a more detailed analysis found in reference 13).

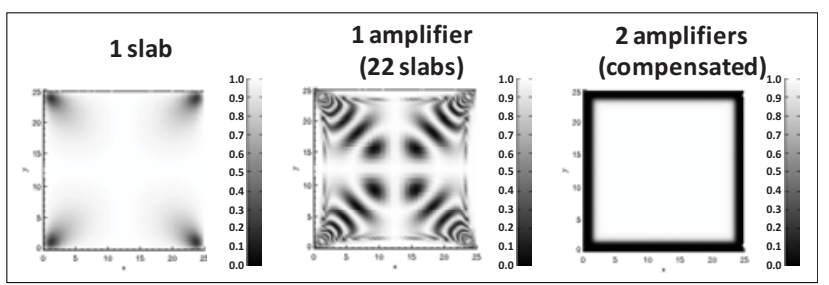

Fig. 6 Results of a depolarization model showing the extreme depolarization loss which would be incurred without compensation, but which can be resolved using a polarization rotator.

\section{III.F. Gain Isolation}

Like Mercury (and unlike NIF), the LIFE architecture is passively switched, requiring no active switch such as a Pockels cell to trap the pulse in the amplifier cavity or to eject the pulse from the cavity. This avoids the need for a high average power Pockels cell, mitigating the difficulty in handling the thermal load associated with residual absorption (see section VII). With a small signal gain of 390,000 , optical isolation is required to prevent parasitic lasing of the entire cavity. By placing a Pockels cell in the location behind the cavity polarizer after two passes through the system, the effective cavity gain for parasitics can be reduced to approximately 625 . The average power loading of the Pockels cell can be reduced to $\sim 20$ times below that required by a cavity switch, which enables the use of simpler, lower cost devices (Section IX).

\section{III.G. Frequency Conversion}

One of the primary design criteria for the LIFE laser system is reliability. Based on damage threshold data for optics at $1053 \mathrm{~nm}$, the maximum average fluence at 1053 $\mathrm{nm}$ is designed to be $15.6 \mathrm{~J} / \mathrm{cm}^{2}$. After amplification, the beam is magnified $1.64 \mathrm{X}$ to lower the fluence into the frequency converter to $5.8 \mathrm{~J} / \mathrm{cm}^{2}$ (including losses). The frequency converters are composed of helium gas cooled highly deuterated $(>98 \%)$ potassium dihydrogen phosphate (DKDP). Gas-cooling is required to remove the heat generated from the $\mathrm{OH}$ absorption in the material. ${ }^{16}$ Simulations of both type I doubler and type II doubler and type II tripler designs based on four crystal cascade conversion using this concept have shown 75\% conversion efficiency (section XI). After conversion, the fluence is $3.9 \mathrm{~J} / \mathrm{cm}^{2}$, which is $\sim 2 \mathrm{X}$ lower than the average $3 \omega$ operational fluence on NIF, which will further enhance the optical lifetime. Recent advances in surface fabrication and mitigation techniques on NIF have enabled a three-fold increase in the damage threshold such that current optics can be manufactured with a countable number of initiation sites (10-20) up to the peak fluence of $12 \mathrm{~J} / \mathrm{cm}^{2}$. These sites can then be mitigated effectively eliminating all initiation sites below $12 \mathrm{~J} / \mathrm{cm}^{2}$, which then becomes a true damage threshold in that no initiation sites will form below this level. ${ }^{17,18}$ Thus operating at $\sim 3.9 \mathrm{~J} / \mathrm{cm}^{2}$ average $\left(\sim 6 \mathrm{~J} / \mathrm{cm}^{2}\right.$ peak), we expect to be far from the point of even creating an initiation (without initiation there is no damage or damage growth). We propose to test this assertion with long term, billion shot level testing in our near term development efforts.

\section{III.H. Final Transport Optics}

The final transport optics represent a fundamental change relative to the NIF laser system. The neutron pinholes shown in Fig. 3 transport the laser beam while significantly filtering neutrons from the target chamber to levels acceptable for human occupation $(\sim 0.04 \mathrm{rem} / \mathrm{yr})$. The neutrons are absorbed by multi-meter thick concrete walls. Relay telescopes puncture these walls to transport the laser beams. In the pinhole region, the void section is essentially the space required to transmit the laser beam with a hole $\sim 1 \mathrm{~cm}$ diameter at the focus. The optic which experiences the greatest set of threats to its lifetime is the final optic. The final optic is directly exposed to the target ignition. The LIFE design provides some benefits 
compared to previous designs. Ions and X-rays are absorbed by the xenon gas in the target chamber. Furthermore, the pressure wave and vibration associated with the gas expansion from ignition and liquid lithium flow in the target chamber blanket are mitigated by the chamber design that mechanically decouples the blanket from the vacuum chamber and optics. The remaining challenge is the $14 \mathrm{MeV}$ neutrons from the fusion events with an average exposure of $1.5 \times 10^{17} \mathrm{n} / \mathrm{m}^{2}$ sec. With a last magnification step $(1.28 \mathrm{X})$, the final optic must efficiently transmit the $351 \mathrm{~nm}$ laser light at $2.4 \mathrm{~J} / \mathrm{cm}^{2}$ and allow high reliability operation, rapid replacement, and adequate MTBF.

The baseline architecture which meets these requirements is a thin Fresnel lens ( $5 \mathrm{~mm}$ fused silica). The Fresnel focuses and deflects the beam to target, preventing radially ballistic neutrons from making it through the neutron pinhole. Irradiation studies of fused silica indicate that the neutron induced absorption in this material saturates to acceptable levels for efficient transmission of the beam to the target (see section XII), although further studies are required to provide adequate data on longevity. The relatively small size and weight of the optic enable its extraction and replacement (compared to, for example a multi-meter grazing incidence mirror). All plant designs require a thin window in this region to act as a gas barrier, and so adoption of a Fresnel to serve as both a gas barrier and focusing element minimizes the optics count and complexity.

\section{SYSTEM MODELING}

Two laser performance codes were used to evaluate the basic four-pass laser architecture chosen for LIFE and to study its possible design variants: an energy-extraction code that uses the Frantz-Nodvik equations ${ }^{19}$ to predict pulse amplification and energy extraction from the $\mathrm{Nd}$ doped amplifier slabs, and a pumping code for predicting gain, stored energy, and waste heat produced by the diode-pumping process. These two codes were designed to scan through large swaths of the design space, to study important design tradeoffs and to identify attractive design options rapidly. A similar approach, in which large regions of the design space were evaluated using similar energetics calculations, was applied during the early design phases of NIF. ${ }^{20}$ Subsequent detailed propagation calculations and experiments provided important design details and tended to confirm results from the initial energetics calculations. $^{21}$

\section{IV.A Extraction}

We used a Frantz-Nodvik ray-trace code to model beam amplification and energy extraction. The amplifier state was described by a single parameter, stored fluence, which is the multiplicative product of amplifier gain in nepers and saturation fluence for the laser transition. Likewise, the state of the laser beam was characterized by a single parameter, the beam fluence. Beam energy was found by multiplying beam fluence by the effective beam area, which were determined from propagation calculations with diffractive effects while using the highfill-factor gain distribution to gain-guide the extracting beam. The mode-fill factor characterizing the size of the extracting beam relative to the pump beam was approximately $92 \%$. The extraction code has been benchmarked against NIF performance and against the propagation codes Prop and MIRO. ${ }^{22,23,24}$

\section{IV.B Pumping}

Pumping of $\mathrm{Nd}^{3+}$ ions by diode light was modeled by numerically integrating a rate equation for excited-state density. Like extraction calculations, pumping calculations were one-dimensional, with a single number characterizing the state of each slab. Separate calculations were performed for each slab within the amplifier slab stack. The rate equation accounted for all important physical processes affecting the excited-state ion density, including absorption of pump light, spontaneous emission, amplified spontaneous emission (ASE), concentration quenching and radiation trapping.

Pump light of equal intensity was assumed incident on each end of the slab stack. Diode emission spectra were modeled as Gaussian distributions with the central wavelength shifting during the pulse to account for thermally-induced chirp, as is characteristic of pulsed edge emitters. The pumping rate was calculated for each slab by integrating the product of the incident diode-light spectrum and the laser-slab absorption spectrum. Filtering effects of previously encountered slabs were included.

Slab absorption spectra were calculated from measured absorption cross sections. The average $\mathrm{Nd}^{3+}$ ion concentration of the slab stack was adjusted so that $99 \%$ of the incident diode pump light was absorbed. Relative $\mathrm{Nd}^{3+}$ ion concentrations of individual slabs were adjusted to equalize excited-state density and gain.

Rates for all important decay processes were included: spontaneous emission, amplified spontaneous emission (ASE), radiation trapping and concentration quenching. While spontaneous emission is directly related to the intrinsic radiative lifetime of a material, ASE tracks the magnitude of the gain and geometry of the amplifier (i.e. this is the parasitic loss associated with high gain). ASE decay rates were based on ray-trace simulation results. The spectral dependence of spontaneous emission, trapping in individual slabs by total internal reflections and slab-to-slab transfer within the closely-packed slab stack were all taken into account. Radiation trapping was estimated as the fraction of the excited-state fluorescence emitted on the resonance transition at $\sim 870 \mathrm{~nm}$ that was trapped by total internal reflection. 


\section{IV.C. Heating and Cooling}

The pumping simulations incorporated heating and cooling effects. Heat sources included waste heat generated by the diodes, thermalizing pump processes within the laser slabs, and absorption of spontaneous emission and ASE by edge claddings surrounding each slab. All electrical power that was not converted to optical output power at the diodes was assumed converted to heat. Waste slab heat was calculated using the quantum defect between absorbed pump light and emitted fluorescence and laser light. Waste heat in the edge claddings included both ASE and the fraction of spontaneous-emission that is trapped by total internal reflection, which were produced both during and after the pump pulse. We accounted for the reduction in excitedstate population caused by the extracting beam.

These simulations provided estimates of the power consumption of chillers, compressors, and pumps, as required for cooling diodes, slabs and edge claddings. Pump power was calculated using pressure drops for specific cooling-channel dimensions and from coolant mass-flow rates needed to limit temperature rise along flow paths.

Slab temperature distributions and tensile stresses at the large faces were approximated using the standard formulas for uniform heat deposition and cooled faces. ${ }^{25}$

\section{IV.D. Beamline Simulations}

Pumping and extracting calculations were performed for the basic architecture shown in Fig. 3, in which two identical amplifiers are four-passed. Beam hard apertures measured $25 \mathrm{~cm} \times 25 \mathrm{~cm}$ for $1 \omega$ and $41 \mathrm{~cm} \times 41 \mathrm{~cm}$ for $3 \omega$, sufficient for producing the required $1 \omega$ and $3 \omega$ energies $(8.1 \mathrm{~kJ}$ and $5.7 \mathrm{~kJ}$, respectively) at operating fluences of $15.6 \mathrm{~J} / \mathrm{cm}^{2}$ and $3.9 \mathrm{~J} / \mathrm{cm}^{2}$ respectively. Margin allows beamlines to produce make-up energy to compensate for non-working neighboring beamlines, thus improving laser availability. Laser slabs were modeled using spectroscopic and thermo-mechanical properties of APG1 laser glass. Slab thickness was set at $1 \mathrm{~cm}$, thin enough for calculated thermal stresses to be well below estimated fracture limits. Amplifiers were modeled with 20 such slabs, each. Using more slabs would lower the average gain coefficient, resulting in smaller ASE losses but greater nonlinear phase shift. On the other hand, using fewer slabs would raise the average gain coefficient, which would increase ASE losses but lower the nonlinear phase shift. We choose 20 slabs for the baseline design to limit nonlinear phase shift to $<2$ radians while minimizing ASE losses. Passive transmission losses now achievable with high-quality optics $(99.95 \%$ transmittance per surface with anti-reflective coatings) were assumed.

The middle, black curve in Fig. 7 shows calculated efficiency versus diode pump pulselength. As diode power increases, pump pulselengths needed to achieve the required gain and stored energy become shorter, decay losses during the pump pulse become smaller, and efficiency increases. As thermal management of the 850watt diode bars becomes more difficult when the duty cycle increases, we have chosen the point corresponding to $164-\mu$ s-long diode pulselengths for our baseline design. Predicted $3 \omega$ electrical-to-optical efficiency is $\sim 18 \%$. The grey curves in Figure 7 represents upper and lower limits for the efficiency predictions, accounting for variability in the design and various inputs used to make efficiency predictions.

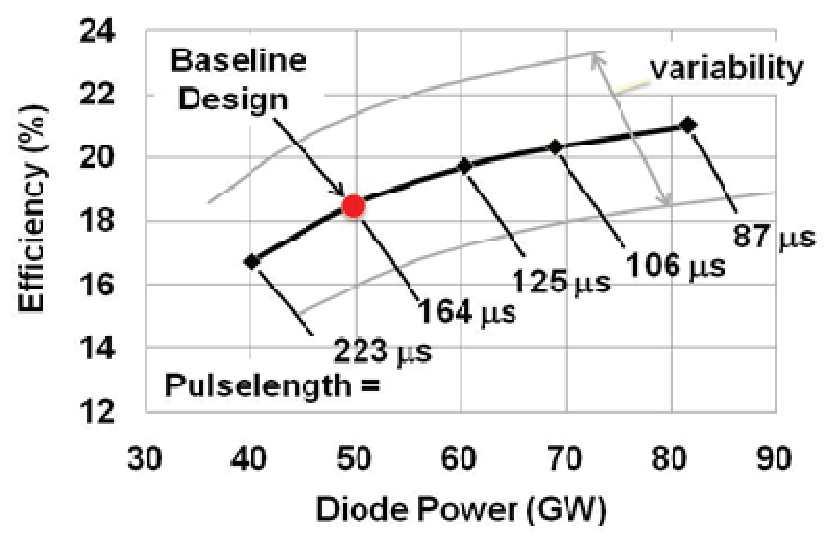

Fig. 7 The LIFE baseline laser design lies on the performance frontier for efficiency vs. power plant diode power, which was modeled over broad ranges of pump pulselength.

Table II lists efficiency and estimated variability for the various energy-transfer processes. Decay losses, extraction efficiency, and passive losses were calculated using the pumping and extraction simulations described above; other efficiency factors were obtained from separate simulations or subject-matter experts. The extraction efficiency is defined as the ratio of extracted energy to the energy that could be extracted at infinite fluence (this efficiency is evaluated in the central portion of the beam, since mode-match factor addresses edge effects). ${ }^{19}$ The "saturation fluence correction factor" is a well-known adjustment to the emission cross section that accounts for a small amount of inhomogeneous broadening present in all Nd:glass laser systems, which is empirically determined from experimental gain saturation / energy extraction data and emission cross section. This factor also includes any non-radiative process which reduces effective gain or extractable stored energy (including excited state absorption, other rare earth impurities, etc.) One of the near term experiments for LIFE is to perform a detailed gain saturation measurement on the APG-1 glass to confirm these numbers for relevant diode pump conditions. Net efficiency results are provided in several formats: $1 \omega$ or $3 \omega$ and electrical-to- 
optical or optical-to-optical efficiencies at the bottom of the table. $1 \omega$ transport losses represent the effective loss to the output beam associated with all of the optics in the $1 \omega$ beamline/amplifier system (note that these passive losses only begin cause an efficiency hit when the amplifiers are extracted which is approximately only on the last pass through the system). $3 \omega$ transport losses include all optics from the frequency converter to the final optic. Some values are derived from the beam propagation models (next subsection) such as the pumplight nonuniformity, which affects the efficiency through a spatially dependent gain to loss ratio during extraction.

TABLE II. Laser system efficiency factors with estimated variability

\begin{tabular}{|l|c|}
\hline Device or Process & Efficiency (\%) \\
\hline DC Power Supply & $95 \pm 2$ \\
\hline Electrical Pulsers & $95 \pm 2$ \\
\hline Diodes & $72 \pm 3$ \\
\hline Diode Micro-Lenses & $98 \pm 2$ \\
\hline Pump-Light Delivery System & $93+2,-5$ \\
\hline Pump-Light Absorption & $99 \pm 1$ \\
\hline Quantum Defect & $83+2,-0$ \\
\hline Spontaneous Emission, Trapping & $85 \pm 2$ \\
\hline Amplified Spontaneous Emission (ASE) & $90 \pm 2$ \\
\hline Saturation Fluence Correction Factor & $90 \pm 4$ \\
\hline Extraction Efficiency & $92 \pm 3$ \\
\hline Pump-Light Non-Uniformity & $99 \pm 1$ \\
\hline Mode-Match Factor (modefill) & $92 \pm 3$ \\
\hline $1 \omega$ Transport & $90 \pm 3$ \\
\hline Depolarization & $99+1,-2$ \\
\hline Frequency Conversion & $75 \pm 3$ \\
\hline $3 \omega$ Transport & $95 \pm 2$ \\
\hline $3 \omega$ Electrical-to-Optical Efficiency & $18 \pm 2$ \\
\hline $3 \omega$ Optical-to-Optical Efficiency & $28 \pm 3$ \\
\hline $1 \omega$ Electrical-to-Optical Efficiency & $25 \pm 3$ \\
\hline $1 \omega$ Optical-to-Optical Efficiency & $39 \pm 4$ \\
\hline
\end{tabular}

\section{IV.E. Beam Propagation}

To understand the 3-D effects of phase errors, Bintegral, depolarization, spatial filtering and related phenomena, 3-D diffractive beam propagation codes were used including the PROP code developed at LLNL, ${ }^{22}$ and the MIRO code developed at CEA in France. ${ }^{23,24}$ Initially, the architecture for the entire beamline was developed in these codes and the basic energetics benchmarked against the energetics models described in this section. To more accurately model the effect of using real LIFE optics, the phase files from similar NIF optics were used. Incorporation of the modeled diode pumped gain profile, thermal phase and depolarization effects are used to simulate the effects of thermal loading and the edge / spatial effects present in the real laser beamline. This simulation approach is expected to provide a fair picture of what to expect from actual LIFE systems. The results of the simulation are shown in Fig. 8 where the $5.7 \mathrm{~kJ} 3 \omega$ output beam is shown to have low contrast $(4.5 \%)$ and the spot size on target meets the LIFE beamline requirements. Contrast is defined as the RMS fluence divided by the average fluence over the central portion of the beam profile. Contrast for the LIFE architecture is improved due to the compact gas-cooled amplifiers ( $\sim 24 \mathrm{~cm}$ path) and strict image relay between each amplifier, which was demonstrated on Mercury to significantly reduce beam modulation. This effect has been verified in comparisons based on propagation models with identical aberrations included. As the optical design and engineering of the LIFE beamline progress, these beam propagation models will provide the basis for benchmarking and optimization of the laser design.

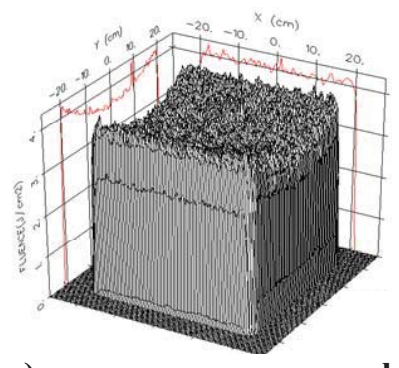

a)

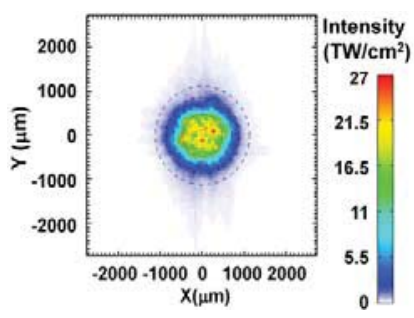

b)

Fig. 8 a) Output beam profile after the tripler producing $=$ $5.7 \mathrm{~kJ}$ with a contrast $\sim 4.6 \%$. b) the output far field at the target location with CPP and Smoothing by Spectral Dispersion (SSD) showing $95 \%$ in a $2.242 \mathrm{~mm}$ spot

\section{DIODE LASER PUMP SOURCE}

Semiconductor diode lasers are used to pump the amplifier slabs to achieve the required laser system efficiency and lifetime. Diode requirements are driven by these considerations, plus the desire to minimize the size and cost of the overall amplifier assembly.

Each amplifier is face-pumped by two planar diode arrays, each delivering $33 \mathrm{MW}$ of peak power at $872 \mathrm{~nm}$ during a $164 \mu$ s Quasi-Continuous Wave (QCW) pulse. The baseline array design employs a $2 \mathrm{D}$ mosaic of submodules, each comprising vertically stacked, $1 \mathrm{~cm}$ wide, edge-emitting diode bars. ${ }^{26}$ The stacks are mounted on a common backplane providing cooling and current drive, to minimize both diode package cost and the number of fluid interconnects for improved reliability (Fig. 9). Edge emitters are used to optimize efficiency, having demonstrated $>70 \%$ wallplug efficiency at wavelengths of $808 \mathrm{~nm}$ and $\geq 940 \mathrm{~nm}$ for Continuous Wave $(\mathrm{CW})$ operation at $60 \sim 1 \overline{6} 4 \mathrm{~W} / \mathrm{bar}$ with low active 
area fill factors. ${ }^{27,28,29} \mathrm{CW}$ output power is limited by thermal and fill-factor constraints, which do not impact the pulsed operation employed for LIFE. Diodes at this wavelength are available near term from nearly all diode vendors due to a well established pump source for $\mathrm{Nd}: Y A G$ at $885 \mathrm{~nm}$. Our design does not preclude future use of surface emitting diodes, ${ }^{30,31}$ which may offer appreciable future cost reductions.

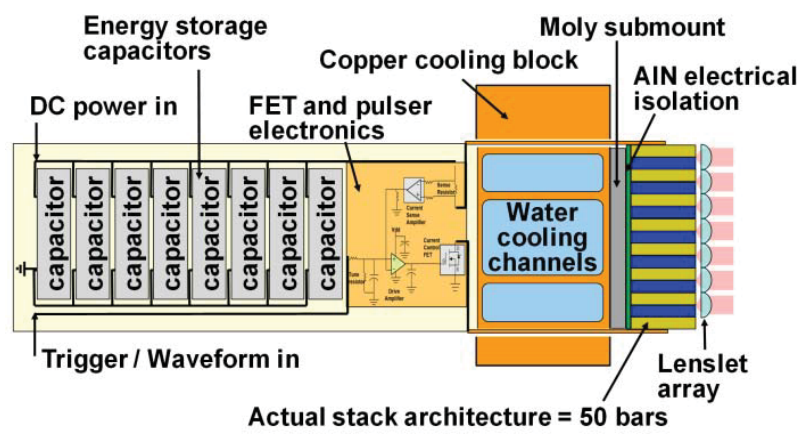

Fig. 9. Schematic view of pump subassembly

\section{V.A. High Power Considerations}

Operation at maximal output power per diode bar is desired to reduce pump costs, which to first order scale with bar quantity. We anticipate operating at $\geq 500 \mathrm{~W} / \mathrm{bar}$, a level below experimental demonstrations of 1000 $\mathrm{W} /$ bar. $^{32,33}$ At $500 \mathrm{~W} / \mathrm{bar}$, several diode manufacturers have concluded that packaged diode stacks can be produced with volume price points of $\$ 0.02 \sim 0.04 /$ Watt for a single plant build, with further decreases to $\$ 0.01$ and below for the additional volume associated with multiple plant builds. ${ }^{34}$ Notably, our face-pumped amplifier architecture avoids stringent diode wavelength specifications, which significantly reduces cost. Efficient absorption is possible since the slab stack effective absorption coefficient can be customized by selecting different neodymium concentrations to provide high absorption (>99\%) for pump wavelengths within a $15 \mathrm{~nm}$ band.

The two challenges for higher power operation are efficiency and lifetime impacts. The higher drive currents required for high bar power can reduce efficiency due to series resistance $I^{2} R$ loss. On chip resistance is minimized by increasing the active bar area, i.e.; increased cavity length $^{3}>2 \mathrm{~mm}$ and active area. Simulations suggest that cavity lengths of $2.4-3.0 \mathrm{~mm}$ can provide efficiencies $>75 \%$ provided that internal diode loss can be maintained at $\sim 0.7 \mathrm{~cm}^{-1}$ (which has been achieved ${ }^{32}$ ). Our design mitigates the resistance of external drive circuitry by maximizing the number of bars (40 50 bars) driven by each driver and by maximally co-locating diodes and circuitry, so that the total stack voltage drop dominates external parasitic voltage drops. Based on measured component resistances within a 700 A prototype driver, ${ }^{35}$ we anticipate that driver series resistance $\leq 10 \mathrm{~m} \Omega$ can be achieved. Future productization of multi-junction diode bars ${ }^{36}$ may further mitigate high current requirements, but are not included in our baseline design.

Diode wearout failure rates are impacted by both operating point power/bar $(\mathrm{P})$ and junction temperature (T).$^{37}$ Current devices at $\sim 300 \mathrm{~W} / \mathrm{bar}$ on $25{ }^{\circ} \mathrm{C}$ heat sinks have demonstrated lifetimes $>3.1$ Giga-shots, ${ }^{38}$ and are expected to exhibit 20 Giga-shot lifetimes. ${ }^{39}$ To mitigate the higher operating power, the diode array is operated with junction temperature near room temperature- $\mathrm{a}$ reduction of $25 \sim 35^{\circ} \mathrm{C}$ over typical operating conditions. This is achieved due to the low duty cycle, a state-of-theart backplane cooling design, and low coolant temperature input to the backplane.

A mini-channel design is used to provide effective backplane cooling without costly and less-reliable microchannel devices. ${ }^{40}$ Using experimental correlations for the minichannels, ${ }^{40}$ simulations show $2{ }^{\circ} \mathrm{C}$ coolant at the backplane inlet holds the junction at $\sim 22^{\circ} \mathrm{C}$.

\section{V.B. Pump Irradiance Considerations}

Achieving a high irradiance $\left(\mathrm{W} / \mathrm{cm}^{2}\right)$ at the array output is important to facilitate size reduction of the overall amplifier and to simplify coupling to the amplifier heads (and reduce coupling optics size). Polarization multiplexing is used to obtain an array irradiance of $\sim 40$ $\mathrm{kW} / \mathrm{cm}^{2}$. Approximately $25 \mathrm{~kW} / \mathrm{cm}^{2}$ is required from the diode stacks, corresponding to a $200 \mu \mathrm{m}$ bar-to-bar pitch for $500 \mathrm{~W} / \mathrm{bar}$ (intensity equivalent to the design point of 340 micron pitch at $850 \mathrm{~W} / \mathrm{bar}$ ), which has been demonstrated experimentally. ${ }^{26}$ The angular acceptance of the polarization combiners $\left( \pm 3^{\circ}\right)$ sets the diode fast-axis collimation requirement. This divergence can be achieved with relatively loose microlens alignment tolerances, suitable for lower cost assemblies. Thermal simulations show that a $340 \mu \mathrm{m}$ pitch does not significantly impact either the diode average junction temperature or the temperature rise of each QCW pulse.

TABLE III. Diode Pump Subsystem Design and Simulated Performance Parameters

\begin{tabular}{|l|c|}
\hline Item & Value \\
\hline Array Peak Power & $\mathbf{3 3 ~ M W}$ \\
\hline $\begin{array}{l}\text { Array Irradiance with } \\
\text { polarization multiplexing }\end{array}$ & $\mathbf{4 0 ~} \mathbf{~ W W} / \mathbf{c m}^{2}$ \\
\hline Pulse width & $\mathbf{1 6 4} \boldsymbol{\mu s}$ \\
\hline Diode wavelength range & $\mathbf{8 7 2} \pm \mathbf{6 . 6} \mathbf{~ n m}$ \\
\hline Average junction temperature & $\mathbf{2 2}{ }^{\circ} \mathbf{C}$ \\
\hline QCW temperature excursion & $<\mathbf{6}^{\circ} \mathbf{C} \mathbf{~ o n ~} \mathbf{C u W}$ \\
\hline Coolant inlet temperature & $\mathbf{2}{ }^{\circ} \mathbf{C}$ \\
\hline Coolant mini-channel dimension & $\mathbf{0 . 5} \mathbf{~ m m}$ \\
\hline
\end{tabular}




\section{V.C. Integrated Pump Subsystem Model}

The key design parameters described above are summarized in Table III. Based on these parameters, the complete pump subsystem (diodes, power conversion, cooling) was modeled to determine its efficiency. The model, which includes cooling pump power and a secondary cooling loop, indicates an overall efficiency for the pump subsystem of $\sim 60 \%$.

\section{DIODE LIGHT DELIVERY}

The diode light delivery system's critical role is to transport the diode pump energy efficiently in a flat profile to the amplifier slabs. Pump delivery designs must address the challenges associated with the distributed, divergent nature of these sources. The LIFE design approach is to create a bright array and minimize the depth over which the light must be delivered. The diode light delivery system for LIFE takes advantage of a high pump irradiance $\left(20.3 \mathrm{~kW} / \mathrm{cm}^{2}\right)$ and low divergence $\left(4^{\circ}\right.$ fast axis $\mathrm{x} 10^{\circ}$ slow axis) source to accomplish this role. It employs polarization combining to achieve additional brightness (Fig. 10a), a common method of increasing pump irradiance used for fiber systems and diode arrays. Polarization combination is accomplished by splitting the array into two sections. A half-waveplate is placed in front of one section which rotates the polarization $90^{\circ}$ such that it transmits through a broadband polarizer ( $p$ polarization). The s-polarized light from the other section of the array is directed by mirror to reflect off the same polarizer such that the combined beams are traveling collinearly. These beams are combined with the collimated fast axis in the plane of incidence to minimize the angular spectrum incident on the polarizer which has a relatively narrow angular acceptance FWHM $\left(\sim 10^{\circ}\right)$. Neglecting loss, the effective irradiance of a given laser source can be doubled. In reality, the optics impose a loss, but this can be managed with proper design. Waveplates and mirrors are low loss items accounting for $<0.5 \%$ to the transmitted intensity. The polarizer in reflection (spolarization) also shows relatively low loss $\sim 0.5 \%$, while in transmission the loss is can be expected to be $4 \%$ or more. The combined transmission is then an average of $97 \%$, or a combined irradiance of approximately 39 $\mathrm{kW} / \mathrm{cm}^{2}$. Note that after polarization combination the pump light is effectively unpolarized, so this method can only be used where the pump polarization is not critical (as in the LIFE architecture). As described in the previous section, there are $1 \mathrm{~mm}$ gaps between the diode stacks for mounting and electrical isolation. In the nearfield, these gaps lead to local regions of low intensity. An additional benefit of polarization combination method is that the two polarizations travel different distances, so they are not strictly relay imaged which tends to blur out the effect of the gaps. Following the polarization combination optics is a simple Keplerian image relay telescope which takes the polarization combined beam as an object and images this diode pump source into the required pump area in the Nd:glass amplifier. The relay imaging provides uniform illumination with high contrast edges as the ray trace model indicates (Fig. 10b). A mirror is used to fold this architecture (for compactness) and direct the output of the telescope through a dichroic which transmits the diode light but reflects the $1053 \mathrm{~nm}$ laser light (Fig. 3). Note that this was the original architecture to be used on the Mercury laser, but low diode brightness prevented the imaging system from being efficient. The polarization combined diodes to be used on LIFE are $\sim 40 \mathrm{X}$ brighter than the Mercury diodes, which make this imaging simple and straightforward.

a)

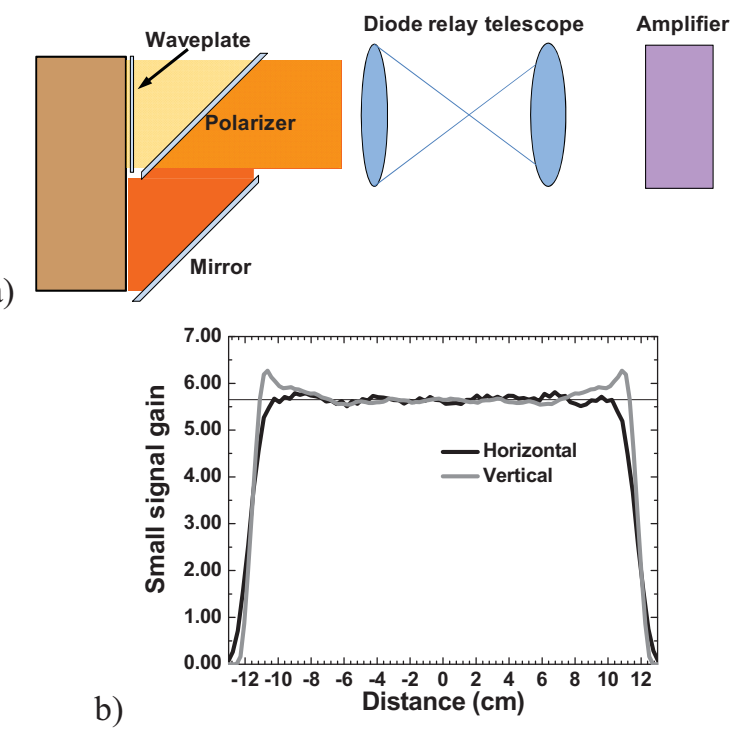

Fig. 10 a) Schematic of pump delivery method showing polarization combination, b) output ray-trace showing homogeneous pump profile

\section{OPTICS AND LASER GAIN MEDIA}

There are a number of different types of optics in the LIFE laser including amplifier glass, lenses, mirrors, windows, a Pockels cell, and frequency conversion crystals. Based on system modeling, these optics specifications have been chosen for manufacturability and to minimize cost. NIF, working in partnership with the optics industry, has developed advanced fabrication processes for more than 90 percent of optics specified for LIFE. While optical lifetime testing at billions of laser shots may ultimately require improvements to the finishing and coating process steps to increase durability, the NIF finishing specifications are currently thought to be adequate for LIFE. The need to adjust optic fabrication steps will be determined by lifetime testing in a one 
hundred $\mathrm{Hz}$ rep-rated laser facility that will be built at LLNL.

The current baseline design for LIFE amplifier gain media is a neodymium-doped phosphate laser glass similar to that used in the NIF and previous laser systems at LLNL. The current NIF amplifier glass and its finishing specifications (i.e., scratch-and-dig limits), require the amplifier slabs to be reduced in thickness to eliminate the potential for surface crack growth due to thermal loading during high-average-power operation. This requires that a 4-cm thick NIF amplifier slab be divided into 1-cm thick slabs. In the LIFE baseline design, the angle of incidence and aperture are also reduced from NIF's from $56^{\circ}$ angle of incidence and $40 \times 80 \mathrm{~cm}^{2}$ slabs to $0^{\circ}$ angle of incidence and $25 \times 25 \mathrm{~cm}^{2}$ thereby improving manufacturability. Advanced glass compositions with higher fracture toughness and thermal conductivity can significantly reduce the potential for thermal fracture. ${ }^{41}$ With improved glass compositions the number of slabs can be reduced to lower overall costs. Anti-reflection coatings will need to be applied to these normal incidence slabs with minima at the pump wavelength $(872 \mathrm{~nm})$ and laser wavelength $(1053 \mathrm{~nm})$. Coatings very similar to those required for LIFE were developed and are used in the rod amplifiers for the NIF preamplifier modules, and a similar coating was applied to the strontium fluorapatite gain medium used on Mercury. As part of the development effort, coating optimization and damage measurements will be performed to engineer this coating to meet LIFE specifications.

The LIFE laser will also require many different lenses, mirrors, and windows. Most of these optics are composed of fused silica which has been fully developed for manufacturability to NIF specifications in sizes larger that the LIFE laser requires. Finishing techniques such as conventional polishing have been used and fully developed in the past, along with Magneto-Rheological Finishing (MRF) and small tool finishing to improve the surface finish and reduce wave-front distortion. In addition, coating techniques have been developed for the NIF facility to meet spectral requirements with a high damage threshold. We expect to maintain the NIF coating specifications in LIFE. It is currently considered that the NIF specifications for optics will be suitable for LIFE and that durability measurements will confirm this to be the case. Therefore, the focus for LIFE on finishing and coating techniques is to reduce the costs by using advancements such as deterministic finishing, automation, or alternative techniques that meet requirements at reduced cost. With regard to surface figure and finishing, the only silica optics which are different from any of the optics in NIF are the cylindrical optics to be used for the spatial filter. While in general cylindrical optics are more difficult to fabricate than spherical lenses, the requirements on these optics (like all optics on NIF) are stringent relative to typical commercial applications and require custom fixturing and test plates regardless of the shape. Given this constraint and the fact that we will need to produce $>1500$ cylindrical optics for even the first LIFE plant, optic vendors have assured us that with proper facilitization, manufacturing will not be a problem when the need arises.

Highly deuterated-potassium dihydrogen phosphate (DKDP) crystals are required for the frequency conversion and Pockels cell optics. NIF requires $70 \%$ deuteration, however, the LIFE laser will require $\geq 98 \%$ deuteration to mitigate heating effects from absorption. The growth of small $98 \%$ DKDP crystals has been achieved, however, the growth of large crystals has not been demonstrated and may be challenging due to the monoclinic phase transition that occurs at lower temperatures as the deuteration level increases. Therefore, development of the technology for growing large highly deuterated crystals will be required. Please note that reasonably large plates of DKDP (50 x $80 \times 10 \mathrm{~mm} 3)$ were fabricated for Mercury frequency converters which showed excellent optical quality and a transmitted wavefront meeting NIF specifications. Thermal aberrations will be discussed in Sections IX and XI. In general these aberrations are very low order wavefront errors (and much smaller in magnitude relative to the amplifiers) due to the face-cooled slab geometry we have adopted which can be corrected with either a static corrector or a deformable mirror.

Two new optical elements relative to NIF are sapphire waveplates and quartz rotators as shown in the LIFE beamline (Fig. 3). These two optic types currently present the highest level of uncertainty for manufacturability and cost. A-plane sapphire crystals are designed for $1 / 4$ waveplates. The blank size of $25 \times 25 \mathrm{~cm}^{2}$ aperture is currently possible by two growth methods; the HEM (Heat Exchanger Method) ${ }^{41}$ and the EFG (EdgeDefined Film-Fed Growth) Method ${ }^{43}$ (Fig. 11). Reducing the cost of the blank optic as well as finishing of this very hard material is necessary for use in the LIFE laser design. Single crystal quartz presents a challenge for producing the size required. Current quartz crystals are grown by the hydrothermal growth method in sizes that produce up to $150 \mathrm{~mm}$ round optics ${ }^{44}$ in a routine production capacity. The scaling of quartz crystals to the necessary size will be a lengthy development path due to the lack of existing seed material of adequate size. Therefore to reduce risk, an alternative method making the large apertures is being pursued which utilizes smaller quartz crystals bonded together to form the required large aperture plate. Once a technique for producing the necessary aperture is developed, cost reduction for polishing and coating will be pursued.

There are potentially alternatives to sapphire and quartz for polarization optics, but these carry even more risk and development. Liquid crystals can be used for polarization control both as waveplates and as rotators, 
but the liquid crystals are organic in nature. There is a risk that at high fluences, the electric field will be sufficient to break bonds. If even a small amount of carbon is formed, runaway damage would occur for large shot count. An alternative to the quartz rotators would be faraday rotators but this would require development of the TGG ceramic rotator material both in size and damage threshold, and magnets which met the size/cost/field uniformity requirements for LIFE. There are of course a whole host of rotary materials for rotators and anisotropic materials for waveplates that could be used. Setting aside damage threshold, and chemical activity of many of these materials, the biggest problem is near term availability in the sizes needed for LIFE. Nonlinear and materials experts have pursued alternative materials for several decades here at LLNL, and the conclusion after this effort is that DKDP is perhaps the only viable material for a Pockels cell. Since development of this material is already required, pursuing alternative materials for conversion is unattractive. Quartz has been used for many years in high energy systems and been proven as a laser material, and from a scale standpoint can already be fabricated in quarter aperture size which makes this material the strongest candidate for a rotator. Likewise, sapphire is already available in full size, with good intrinsic damage threshold. Remaining development lies in minimizing fabrication cost, and optimizing the surface fabrication for highest damage threshold.

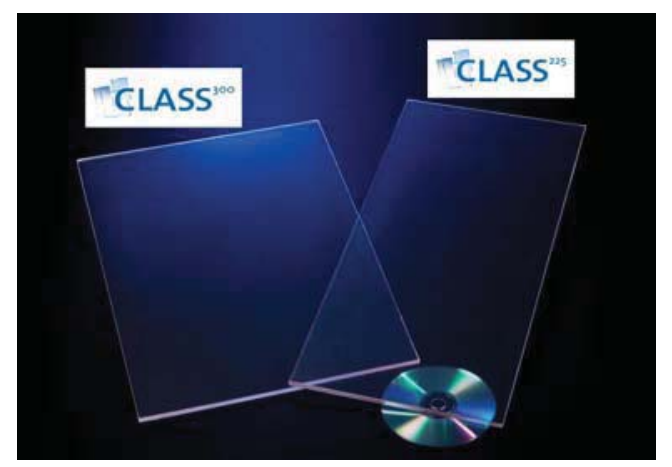

Fig. 11. Edge-Defined Film-Fed Growth (EFG) sapphire plate from Saint Gobain showing current capability of 1.0 x $30 \times 40 \mathrm{~cm}^{3}$ and $1.0 \times 22 \times 60 \mathrm{~cm}^{3}$

\section{COOLING SYSTEMS}

The two primary laser cooling systems are a liquid (water-based) cooling loop for general cooling and a high pressure Helium gas loop for the amplifier heads. The liquid loop cools the diode pumps, Helium gas, and assorted additional subsystems (e.g; beam dumps). It accounts for $\sim 90 \%$ of the electrical power consumption in the cooling subsystem, which is dominated by chillers. Cooling subsystem costs are divided approximately equally between the liquid and Helium loops, with the gas loop cost being dominated by high pressure compressors used to drive the Helium flow.

The primary heat load on the liquid loop is due to the pump diodes (diode cooling details are provided in Section IV). The primary coolant is chilled using heat exchangers interfaced to a secondary cooling system/chiller using ammonia as the working fluid.

Amplifier heads are cooled by flowing high pressure (5 atm.) helium gas over the amplifier slab faces at $\sim 50$ $\mathrm{m} / \mathrm{s}$, in a face-cooled configuration that minimizes thermal birefringence. This approach uses Helium gas to avoid beam quality degradation, ${ }^{8}$ and was previously demonstrated in a similar high energy/high average power beamline. ${ }^{6}$ The Helium cooling loop external to the amplifier heads comprises a compressor and a distributed set of intercoolers. Suitable industrial scale, high reliability compressors are available from several manufacturers. Each amplifier head is served by a separate, water-cooled heat exchanger/intercooler, so that multiple heads can be cooled in series by a single compressor and the overall subsystem cost can be optimized by increasing the number of beamlines served per compressor. For maximal availability, bypass ducts are deployed in the helium plumbing so that gas flow can be rerouted around any beambox that must be replaced. This enables other beamlines sharing a common compressor to continue operations in the event of failure or replacement of one beam box.

\section{GAIN ISOLATION}

Because polarization rotation is used to switch pulses out of the amplifier, the gain isolation device need not be located at the output of the amplifier cavity. It can be placed in a lower fluence location, and in our design this fluence is $<0.4 \mathrm{~J} / \mathrm{cm}^{2}$. This low fluence opens up a wider range of design options for the gain isolation device, from which we have selected a longitudinal Pockels cell based on a z-cut, highly deuterated potassium dihydrogen phosphate (DKDP) crystal. We use a longitudinal configuration to reduce switching voltage and avoid the need for stringent crystal matching (to compensate thermal birefringence), as compared to transverse devices. ${ }^{45}$ DKDP is used as the electrooptic material due to its low optical loss and availability in large aperture.

The implementation challenge for longitudinal Pockels Cells is the need for a transparent electrode in the optical beam path. In a high energy fusion laser, this electrode must withstand repetitive exposure to high energy pulses without degradation. An elegant solution proposed for the National Ignition Facility employed low pressure plasmas for these electrodes. ${ }^{46}$ Such plasma electrode devices, however, provide very little ability to cool the DKDP crystal, and could present difficulties in high average power applications. A potential alternative is the use of transparent, conductive thin film electrodes 
based on materials such as indium tin oxide (ITO), ${ }^{47,48,49}$ which has been demonstrated with optical damage thresholds in the $3 \sim 11 \mathrm{~J} / \mathrm{cm}^{2}$ range. ${ }^{47,48}$

It is challenging to achieve both high transparency (a prerequisite for high damage threshold) and low resistivity for ITO that is directly deposited on DKDP. Because DKDP has a destructive phase transition near $145{ }^{\circ} \mathrm{C}$, the ITO deposition must be performed at relatively low substrate temperatures, resulting in high sheet resistance $(>200 \Omega$ /square) for high damage threshold films. ${ }^{47,48}$ The high sheet resistance, in combination with the crystal capacitance, forms a diffusive delay line with a slow rise time that is unsuitable for gain isolation on timescales of $\sim 100 \mathrm{~ns}$.

For this reason, we have selected an alternative approach in which the ITO is deposited on a separate substrate, which is positioned in close proximity to the DKDP crystal (Fig. 12).

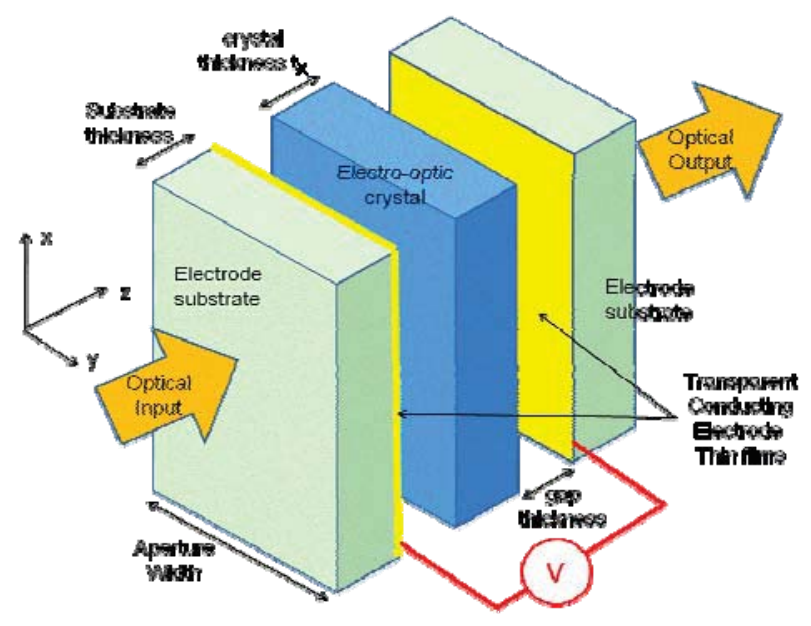

Fig. 12. Schematic view of gap-coupled Pockels Cell

By using a high-temperature substrate such as sapphire, the optimal electrode deposition temperature (near 300 ${ }^{\circ} \mathrm{C}$ ) can be used to optimize the transparency/resistivity tradeoff. The thermomechanical properties of sapphire are also attractive for this application. The high thermal conductivity-heat capacity product of sapphire significantly reduces the ITO thermal rise due to residual absorption of laser pulses, and the close expansion coefficient match of ITO to sapphire reduces the stress induced by transient laser pulse heating. Based on the performance achieved for ITO deposited under optimized conditions, we anticipate that electrodes can be achieved with $\sim 1 \%$ absorption loss, $\sim 150 \Omega$ /square sheet resistance, and damage thresholds $>1.5 \mathrm{~J} / \mathrm{cm}^{2}$.

\section{IX.A. Cooling Considerations}

At high repetition rates and moderate fluence, low level absorption within the DKDP causes nonuniform heating of the crystal. This raises concerns with crystal fracture or thermal birefringence (which degrades gain isolation). Our designs limit the center-to-face temperature gradient within the DKDP to $<1{ }^{\circ} \mathrm{C}$ to avoid fracture concerns, and employ face cooling of the crystal to minimize thermal birefringence effects. ${ }^{45}$ The gap coupled electrode structure establishes a channel for fluid flow between the crystal and electrode surfaces, providing a natural mechanism for face cooling. This can be achieved using either liquid or high pressure gas coolants. With gas cooling, dielectric breakdown of the gas is a concern, forcing the design to thicker crystals (increased heating and thermal birefringence) and high gas pressures ( $\sim 2.5 \mathrm{~atm}$, thick windows). For these reasons, an organic liquid similar to those employed in commercial systems will be used. Development and demonstration of this Pockels cell technology is currently underway.

\section{IX.B. Design and Simulated Performance}

The electrode-to-crystal gap spacing is a key design parameter, since it sets the drive voltage, device capacitance, and coolant pressure drop. The baseline design employs $8 \mathrm{~mm}$ thick DKDP, $0.4 \mathrm{~mm}$ gaps, and $26 \times 26 \mathrm{~cm}^{2}$ device area. This provides a halfwave voltage of $20.8 \mathrm{kV}$, appreciably less than that required for transverse devices at the same aperture $(>50 \mathrm{kV})$, and 1.3 $\mathrm{nF}$ capacitance. The electrode-resistance-limited risetime is $70 \mathrm{~ns}$ to $2 \%$ settling for $150 \Omega$ /square sheet resistance.

This configuration can be cooled with a $0.24 \mathrm{~m} / \mathrm{s}$ coolant flow in the gaps, requiring 1 psi pressure drop. Under these conditions, the temperature variation within and across the DKDP is $<0.3{ }^{\circ} \mathrm{C}$, and the crystal temperature rises $2{ }^{\circ} \mathrm{C}$ above the coolant due to boundary layer effects. This results in a simulated extinction ratio degradation due to thermal birefringence below $0.1 \%$.

\section{BEAM CONTROL}

From an alignment perspective, LIFE has significant architecture similarities to NIF. Like NIF, the laser is an image-relayed, four-pass-amplifier design; the pulses propagate to the target area through a system of transport optics; and each beam is precisely aligned to a specific point on the target. ${ }^{50,51}$ However, there are also some important differences: LIFE operates continuously at $16 \mathrm{~Hz}$, while NIF is a single shot system; pump light as well as the pulsed beam must be aligned; the output from the fusion engine places new limitations on the location of control electronics; and finally, each pulse must hit a moving target. It is important to note that shooting this small target is not outside demonstrated commercial capabilities. Note also the large difference in speed. The target is moving at $200-400 \mathrm{~m} / \mathrm{s}$, while the tracking system is operating at the speed of light $3 \times 10^{8} \mathrm{~m} / \mathrm{s}$. The target will look frozen in space relative to the light 
diagnostics and pointing. Taking into account time required for detection, controls for repointing, and laser triggering $(\sim 17 \mu \mathrm{s})$, the target only moves about $7 \mathrm{~mm}$ (and this distance is readily precompensated).

For LIFE, a slow pointing and centering subsystem $(\sim 1 \mathrm{~Hz})$ maintains optimum average alignment throughout the facility by compensating for low frequency drift and for the thermal changes associated with the full range of start up and normal operating conditions. The effects of ambient vibrations from laser cooling, maintenance activities, power plant components, and local ground motion are controlled by a combination of mechanical design and closed-loop pointing compensation. Mechanical resonances are required to be $50 \mathrm{~Hz}$ or higher and to avoid $\mathrm{AC}$ power frequencies. Higher frequency pointing changes tend to be smaller in amplitude and are compensated by a fast pointing system with a closed loop bandwidth of hundreds of $\mathrm{Hz}$. The operation of this system is tuned to maximize pointing stability across the full frequency range. These functions are noted in Fig.13.

\section{X.A. Target Tracking and Engagement}

A new capability for LIFE is target tracking and engagement. Target tracking measures the trajectory of each target as it approaches the nominal shot location. Orthogonal pairs of horizontal beams cross the target path at several vertical positions. At each level, opposing sensors detect the time and transverse location of the passing target by its effect on the transmitted beam.
Analysis of this data predicts the precise location and arrival time of the target in the shot zone.

Immediately before the ignition pulse is injected into the amplifiers, the engagement subsystem measures and refines the pointing of each beam line with respect to the target. Separate pulsed engagement illuminators (dashed line in Fig. 13) provide a backward propagating glint from the target. Comparison of the glint light direction with that of the forward-going laser alignment beam determines the final laser pointing correction, which is implemented with acoustic beam deflectors in the small aperture front-end of the laser system. Note that this deflection is small (approximately $50 \mu \mathrm{rad}$ deflection in the LIFE amplifier) relative to the size of the spatial filter opening in the relay telescope between the LIFE amplifiers (approximately $600 \mu \mathrm{rad}$ ).

LIFE beam diagnostics perform the same basic functions as on NIF. They monitor the repeatability of pulse energy and temporal shape because these properties must be within tolerance for each target. They also measure beam average power, transverse profile, and frequency conversion efficiency as inputs to maintaining optimum beam line performance. An additional subsystem monitors the condition of the final optics, which are exposed to a more stressful environment than the rest of the system The sensors required to implement Beam Control functions are shared and integrated to the maximum possible extent to minimize cost, space, computer control overhead, and maintenance requirements.

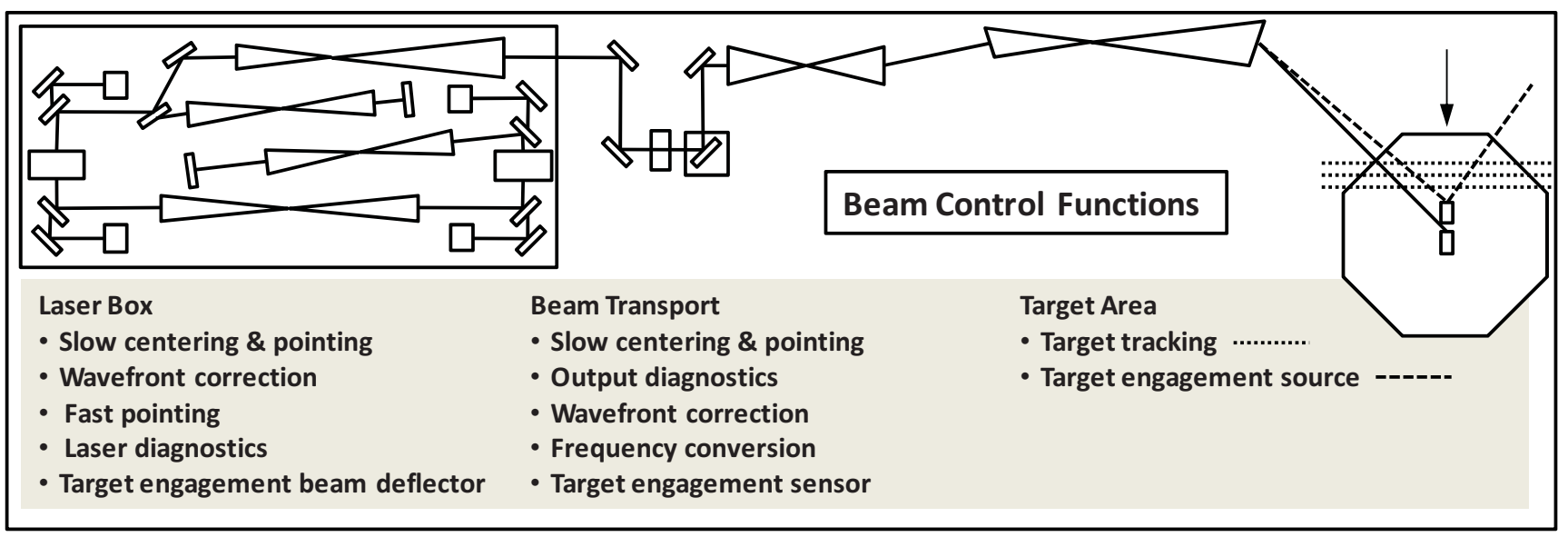

Fig. 13 Beam control schematic diagram

\section{X.B. Wavefront Correction}

The NIF wavefront correction system successfully compensates for both the static aberrations of beam line components and the thermal distortion introduced by firing the main beam amplifiers. ${ }^{50}$ LIFE requires a similar capability, but its amplifiers introduce significantly more thermal distortion at the beam edges. This occurs because the size of the pumped footprint is closely matched to the beam size for maximum efficiency and produces large index gradients at the edge. A static wavefront corrector is incorporated within each amplifier to compensate for this effect. These correctors are easy to fabricate using the same technique used to make the CPPs - namely 
Magneto-Rheological Finishing (MRF), a deterministic polishing technique that allows the user to literally program a desired surface into a computer controls on the MRF machine which then creates the optic with high fidelity and high damage threshold. This particular method (corrector vs. CPP) was successfully used on the Mercury laser system and enabled much of the early operation of the system. ${ }^{6}$ Alignment of these plates with simple micrometer stages was straightforward because of the large length scale of thermal aberrations $(\sim 3-10 \mathrm{~mm})$. The open architecture of the LIFE amplifiers will allow an extremely simple, robust, and accurate method of alignment: the pumped area can be aligned to a corrector centered in the amplifier through simple feedback between beam diagnostics and the pump alignment system. Once aligned, this system will only have to account for long term drift. The residual wavefront error and other long term thermal drifts are compensated by a full aperture deformable mirror controlled in continuous closed loop $(1 \mathrm{~Hz})$.

\section{FREQENCY CONVERSION}

Frequency conversion ${ }^{52}$ of the fundamental wavelength at $1053-\mathrm{nm}$ to the second and third harmonic wavelengths of $527-\mathrm{nm}$ and $351-\mathrm{nm}$ allows for more efficient absorption of laser energy by the target. ${ }^{53}$ This section describes a method for efficiently converting the desired pulse shape to short wavelengths using highly deuterated potassium dihydrogen phosphate crystals (DKDP). Highly deuterated DKDP is desirable for highenergy, high-average-power frequency conversion because of its potential low absorption. ${ }^{16}$ Sapphire or fused silica windows surrounding the DKDP crystals form cooling channels for flowing Helium gas using the same method as with the laser amplifiers. The continuousphase-plate (CPP) for beam smoothing applications at the target plane, would be located midway between the doubler and tripler crystal pairs (not shown), and will have no impact on the conversion efficiency (as we have shown with detailed simulations).

\section{XI.A. Pulse Shape}

The third harmonic pulse delivered to the target is divided into two portions known as the 'foot' and 'drive,' as shown in Fig. 14. The foot and drive portions each traverse separate amplifier and frequency converter modules. Since the foot and drive have similar peak intensities, a frequency converter can be designed to reach optimal efficiency as compared to frequency converting the "total" pulse with one converter. One "foot" beam line is required for three "drive beam lines, and the focal planes of each must overlap on the target for the integrated pulse shape to match that required by ICF target physics. For interchangeability in the LIFE facility, the "foot" and "drive" beam converters are optimized with the identical crystal phase-match types and thicknesses. The implications of this are that a particular beamline is flexible to be either a foot pulse or a drive pulse on the fly. Since the pulse shape for each beam box is controlled by its PAM, if replacement of the one of the small number of boxes producing a foot pulse is required, a box producing a drive pulse can be switched to a foot pulse (or any in-between shape that is deemed necessary during the maintenance). This is accomplished using preprogrammed pulseshapes which are loaded into the computer controls, where pulse switching has been demonstrated in Mercury lab to be possible even on the next shot.

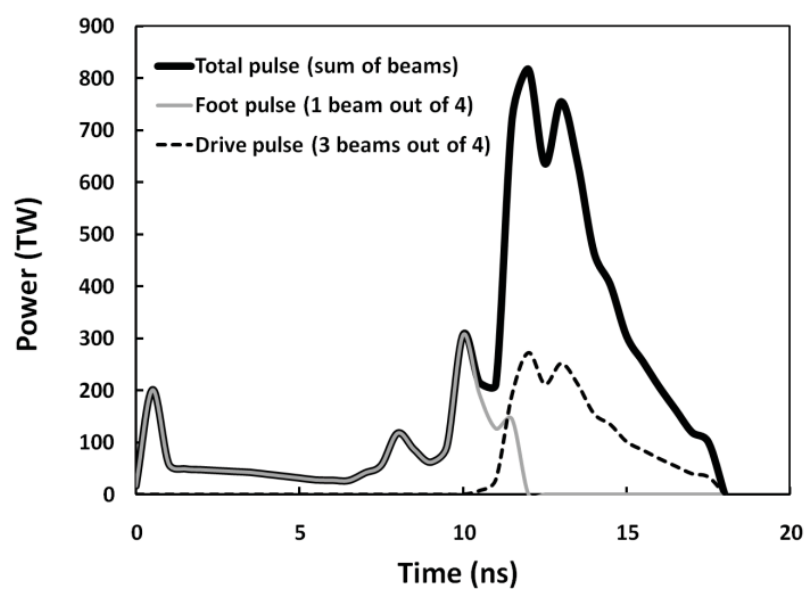

Fig. 14. The pulse shape is shown with its respective foot and drive portions for optimizing conversion efficiency.

\section{XI.B. Frequency Converter Design}

Conversion to the second and third harmonics is accomplished via a cascaded 4-crystal design, ${ }^{54}$ two DKDP crystals each for second and third harmonic conversion, as shown in Fig. 15. For frequency doubling and tripling, the two crystals are rotated 180 degrees about the beam-propagation axis with respect to one another, creating an alternation in the c-axis orientation. ${ }^{55}$ This scheme is referred to as alternating-z. However, parallel z-axis operation is also possible, if attention is paid to the alternation of sign in the doubler angle-tuning. These four-crystal schemes require thin crystals, which are ideal for thermal and stress management under high average power operation. They also allow a wide range of incident intensities to be efficiently converted, thereby increasing the dynamic range, ${ }^{55}$ as compared to twocrystal frequency converter designs (not shown).

Type I or type II phase matching is considered for the second harmonic or 'doubler' crystals, while type II phase matching is used for the third harmonic or 'tripler' crystals. ${ }^{52}$ Type I phase matching is preferred for the 
doubler since the four crystal type I/type II scheme has near-equal conversion efficiency for the LIFE pulse shape shown in Fig. 14 compared to Type II phase matched doublers and triplers ( $76.5 \%$ vs. $76.3 \%$, respectively), and does not require an additional 35.3 deg rotation of the input fundamental beam polarization. Also, type I/type II tripling is the only phase-match scheme in DKDP that can tolerate a moderately non-uniform polarization of the $1053-\mathrm{nm}$ beam. ${ }^{52}$ For that reason, it has become the default choice for ICF lasers around the world. In the LIFE laser design, aggressive birefringence compensation will limit polarization non-uniformities, allowing for efficient frequency conversion with either a Type I/Type II or Type II/Type II frequency tripling scheme.

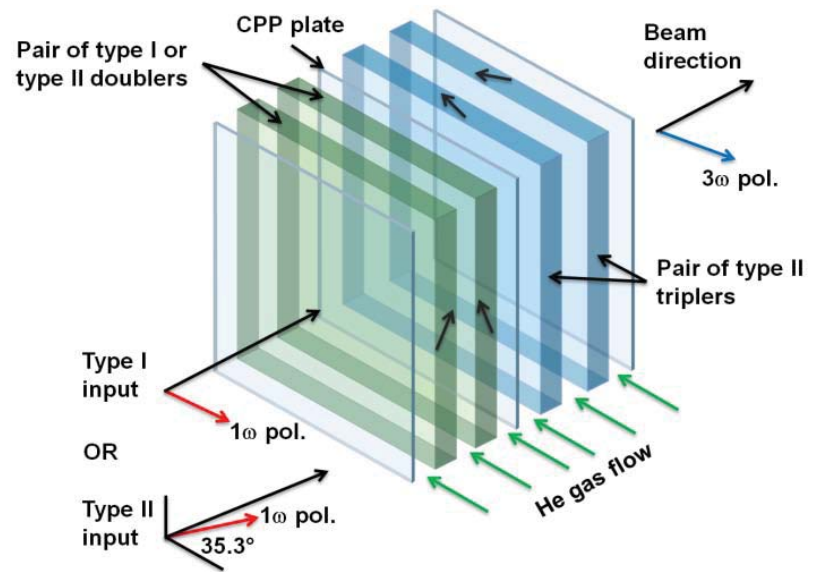

Fig. 15. The frequency converter with two DKDP crystals for frequency doubling and tripling is shown. Type I phase matching requires horizontally polarized input. Type II phase matching requires the linearly polarized beam be oriented at 35.3 degrees.

For Type I phase matching, the input polarization is linear and along the ordinary axis of the first doubler. For Type II phase matching, on the other hand, the incident fundamental beam is polarized at 35.3 degrees with respect to the ordinary axis of the first doubler crystal. The ratio of incident fundamental power along ordinary and extraordinary axes is 2 to 1 . This provides the optimal frequency mix ratio of fundamental and second harmonics to produce the third harmonic in the pair of tripler crystals. The use of Type II doublers would require a quartz-rotator or sapphire half-wave plate upstream from the converter, or else crystal orientations that are rotated 35.3 degrees about the propagation direction. In Type I phase matching, the optimal mix ratio of fundamental to second harmonic for tripling is obtained by angle-tuning the phase-match error (with opposite sign) in the doubler crystal pair. Further study is needed to decide which phase-matching approach is more robust against thermal distortion and ultimately less expensive.
In our simulations, slight angular detuning in the triplers of approximately $30 \mu \mathrm{rad}$ from exact phase matching is allowed to account for errors in crystal manufacturing. The sign of the angular tuning error must be adjusted depending on the crystal's alternating-z orientation. Additionally, our simulations include the phase-mismatch effects of $60 \mathrm{GHz}$ of FM bandwidth for the fundamental beam $(180 \mathrm{GHz}$ at the third harmonic), required for beam smoothing and suppression of transverse stimulated effects. ${ }^{1}$ Increased third harmonic bandwidth capability (up to $450 \mathrm{GHz}$ ) might also be achieved, if necessary for target physics, by appropriate phase-mismatch (by opposite angle tuning) of the tripler crystal pair. ${ }^{56,57}$

Frequency converter parameters and efficiencies are listed in Tables IV and V. For a given third harmonic pulse shape, the incident pulse shape and required input energy are calculated at the input of the laser amplifier and frequency converter chain. Diffraction and beam quality are accounted for in the 1053-nm laser chain and in the frequency converter. Conversion efficiency can be optimized by changing the crystal lengths and angular tuning. While converter designs can be separately optimized for the "foot" and "drive" beams, the four crystal type II/type II design listed in Table IV uses crystal lengths of 8 and $9 \mathrm{~mm}$. In the type I/type II scheme, we've used a thicker first doubler, following the work of Eimerl. ${ }^{55}$ However, for best thermal management and reduced risk of crystal fracture, a thinner first doubler may be used - by reversing the order shown in Table V with only a few percent loss in conversion efficiency. Further crystal thinning is also possible with some loss in efficiency. Adding a third doubler and/or tripler crystal is also possible. While our detailed modeling of thermal aberrations is still in progress, we anticipate longitudinal thermal excursions of approximately 1 degree $\mathrm{C}$ for highly deuterated KDP (>98\%), which should allow the conversion efficiency to remain high.

TABLE IV. Frequency Converter Parameters for a Four Crystal Type II/Type II Tripling Scheme

\begin{tabular}{|l|c|c|}
\hline & Foot Portion & Drive Portion \\
\hline Crystal lengths $(\mathrm{mm})$ & $\mathbf{9 , 8 , 8 , 9}$ & $\mathbf{9 , 8 , 8 , 9}$ \\
\hline Angular detuning $(\mu \mathrm{rad})$ & $\mathbf{3 0 , - 3 0 , 3 0 , - 3 0}$ & $\mathbf{3 0 , - 3 0 , 3 0 , - 3 0}$ \\
\hline Conversion Efficiency & $\mathbf{6 9 . 5} \%$ & $\mathbf{7 8 . 5} \%$ \\
\hline
\end{tabular}

TABLE V. Frequency Converter Parameters for a Four Crystal Type I/Type II Tripling Scheme

\begin{tabular}{|l|c|c|}
\hline & Foot Portion & Drive Portion \\
\hline Crystal lengths $(\mathrm{mm})$ & $\mathbf{1 3 , 1 1 , 8 , 1 1}$ & $\mathbf{1 3 , 1 1 , 8 , 1 1}$ \\
\hline Angular detuning $(\mu \mathrm{rad})$ & $\mathbf{2 8 0 , - 1 8 0 , 3 0 , - 3 0}$ & $\mathbf{2 8 0 , - 1 8 0 , 3 0 , - 3 0}$ \\
\hline Conversion Efficiency & $\mathbf{6 8 . 9} \%$ & $\mathbf{7 9 . 0} \%$ \\
\hline
\end{tabular}




\section{FINAL BEAM TRANSPORT SYSTEM}

The final beam transport system encompasses all of the optics required to transport the beam from the exit of the frequency converter to the target chamber center. In addition to the laser fluence at $351 \mathrm{~nm}$, several of these optics are exposed to neutron irradiation, and the final optic to additional mechanical shock and target shrapnel from target ignition. This final optics transport shown schematically in Fig. 3, is fundamentally different than the NIF architecture which essentially has a wedged focusing lens and debris shield installed in close proximity to the frequency converter. To protect the laser system and operations personnel from neutron irradiation, devices called neutron pinholes are used. The neutron pinhole is a small $(\sim 1 \mathrm{~cm})$ hole in the meter thick concrete shield walls which allow light to pass, but also neutrons. If this pinhole is situated at the focal location of a Keplerian relay telescope, the aperture of this pinhole can be minimized (theoretically to the same size as pinholes in the $1 \omega$ beamline) thereby minimizing transmitted neutrons while fully transmitting the laser light. Calculations based on the hardware shown in Fig. 16 indicate that the radiation dose can be attenuated to 0.04 $\mathrm{rem} / \mathrm{yr}$. utilizing two cascaded telescopes. Note that the final optic not only focuses but deflects the beam from the axis of the final neutron pinhole relay telescope to the target chamber center. While the final optic deflects the laser beam, it can only act as a scattering source for neutrons thereby preventing ballistic neutrons from passing through the neutron pinhole. The transmitted spectrum of neutrons from the pinhole will be a roughly Lambertian source of neutrons which have scattered from the surrounding shield materials and blanket. The axis of the first neutron pinhole is orthogonal in the architecture shown in Fig. 16 which prevents ballistic neutrons from the first pinhole from passing through the second one.

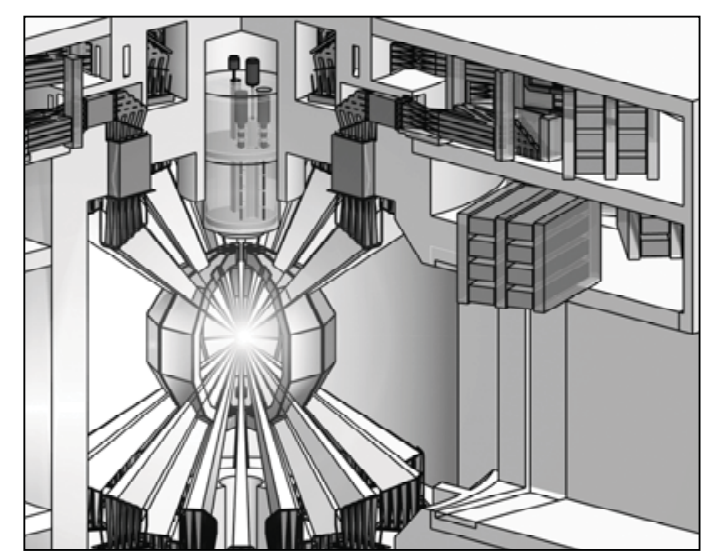

Fig. 16 Target chamber and beamlines showing final optic focusing and two cascaded neutron pinhole relay telescopes
As indicated in the architecture section, the optic which is the most at risk in the entire laser system is the final optic. The final optic is directly exposed to the target chamber gases (primarily xenon, but with target admixture of helium, hydrogen, deuterium, tritium, carbon etc) and target shrapnel. The baseline output of the LIFE.2 (first commercial) power plant is $2100 \mathrm{MW}$. The ions and x-rays are absorbed by the xenon gas in the target chamber, leaving $1730 \mathrm{MW}$ of $14 \mathrm{MeV}$ neutrons from the fusion reaction which yield an average exposure of $1.5 \times 10^{17} \mathrm{n} / \mathrm{m}^{2} \cdot \mathrm{sec}$ at the final optic location. In addition there is a pressure wave producing $\sim 0.53$ torr of pressure at the final optic location in addition to the baseline pressure of 21 torr. Finally, the optic sits in an environment coupled to the vibrations associated with the gas expansion from ignition and liquid lithium flow in the target chamber blanket. This is somewhat mitigated in the LIFE design by mechanically decoupling the first wall and blanket from the vacuum chamber that is connected to the optical pipe assembly. The beamline apertures in the blanket also act to attenuate the gas shock incident on the final optic. The final optic must survive the residual threat and efficiently transmit and focus the $351 \mathrm{~nm}$ laser light at $\sim 3 \mathrm{~J} / \mathrm{cm}^{2}$ (normal to the beam).

There are several possibilities for the final optic including: a grazing incidence metal mirror (GIMM), a parabolic mirror, and a thin Fresnel optic. Note that if the GIMM or parabolic mirror is used an additional vacuum window must be included in the design immediately upstream of the final optic before the neutron spatial filter. This optic serves two purposes: first to guarantee vacuum at the telescope focus so that the laser light can be transmitted, and to serve as a tritium barrier. Careful study of the different options indicates the Fresnel optics offers the lowest risk at this time. The GIMM mirrors by necessity will be very large making them difficult to fabricate, mount, point and/or correct wavefront. The metal substrate of the GIMM, like all metals has a low damage threshold and can only achieve a high threshold by having a nearly atomically flat surface. Such a system is perturbation unstable to even the smallest of shrapnel particles from the target. The parabolic mirror is difficult to mount and point in this environment, and the dielectric coating on the surface is extremely sensitive to modification which could arise from the radiation. Further study is needed to determine the magnitude of these effects on the reflectivity and the damage threshold. Both the GIMM and the parabola also require the vacuum window which essentially represents another optic in the high radiation environment (doubling the risk). The Fresnel optic can act as both the final focusing optic and as the vacuum barrier. By making this optic thin, the neutron induced absorption can be reduced to the few \% level. Based on earlier work ${ }^{58,59}$, the neutron induced absorption in fused silica saturates at fairly modest neutron irradiation levels, and this absorption can be 
partially annealed by raising the temperature of the substrate. A $5.3 \mathrm{~mm}$ thick fused silica substrate for the Fresnel optic would be sufficient to serve as the vacuum barrier between the target chamber at 21 torr and the relay telescope at $\sim 100 \mu$ torr. Simulations based on the experimental data indicate that if this $5.3 \mathrm{~mm}$ thick optic were maintained at $\sim 580{ }^{\circ} \mathrm{C}$, the absorption loss could be reduced to $\sim 0.5 \%$. The heating can be accomplished through a combination of the beam heating and an external heater producing $\sim 3.4 \mathrm{MW}$. If no heater is used, the beam heating alone will raise the temperature of the optic to $\sim 518{ }^{\circ} \mathrm{C}$, with associated transmission loss of $~$ $3.5 \%$, which is still reasonable. For these reasons, a $5 \mathrm{~mm}$ thick fused silica Fresnel optic is the current baseline architecture for the final optic. The damage threshold of this optic is also in question and will require further study to determine the combined effect of these threats on the lifetime of the optic. While almost all of the LIFE systems can be developed separately, the final optic (and all other materials which must withstand the heavy neutron flux) must be tested in an integrated facility like the proposed first LIFE power plant.

The final issue which is shared by all final optic options and by the other optics between the two neutron pinholes is the replacement of these optics in a radiation hot environment. To first order all electronics cannot survive in this environment and would have a low MTTF. Hydraulics and/or simple mechanical solutions are being considered. At this time, an engineering solution has been identified which is being modeled and will be demonstrated with a engineering prototype to prove out the capability. The replacement hardware must have a very large MTTF since failure of these components would require plant shut-down (affecting plant availability) to enable access to the hardware in the high radiation area around the target chamber. Our solution puts all of the short lived components on the final optic LRU itself, so that the MTTF of anything which is not accessible is commensurate with plant lifetime. This architecture is robust against catastrophic failure of the Fresnel final optic since upstream optics act as secondary and tertiary barriers. The vacuum system handling the both neutron pinholes is connected to the tritium handling system in the event of a leak during failure or intentional leak during optic replacement. Finally the optic is monitored with a damage detection system similar to that present in the $1 \omega$ beamline and demonstrated on Mercury which evaluates the appearance of any flaws in the optic and shuts down the beamline as necessary.

With a final optic solution identified, a complete optical model of the final beam transport system has been constructed. Grating efficiency codes are used to define the operational space of the Fresnel optic. Transmissive Fresnel optics have optimal efficiency for deflection angles between 36-68 degrees when operated near Littrow angle. While the beam will not experience any anamorphic change in this orientation, the models predict a large temporal skew in the pulse as well as chromatic aberration which causes the far field spot size to increase. To minimize the negative chromatic aberration in the Fresnel optic, a refractive relay lens (L9) with positive chromatic aberration was added to balance the entire system enabling chromatic performance similar to NIF. Likewise, a second diffractive optic (grating LG in fig. 3) was introduced to compensate for temporal shear induced by the Fresnel optic across the pulse. This grating serves double duty as a second neutron resistant optic since it is the next optic in the path of neutrons scattered by the final optic through the neutron pinhole \#2 (see Fig. 3). This grating can also be made thin and take advantage of dramatically reduced neutron threat relative to the final optic. To improve focal spot size at the 17 meter standoff and reduce the fluence load to the final optic, the last telescope magnifies one last time by a factor of $1.28 \mathrm{X}$ and image relay is set at the final optic location which reduces the average fluence on the final optic to $2.4 \mathrm{~J} / \mathrm{cm}^{2}$ with contrast similar to the frequency converter $(\sim 4.5 \%)$. Due to the scarcity of neutron sources for exposing large areas of grating material for damage tests, ion bombardment will be used for near term evaluation of radiation on the grating geometry. As stated earlier, bulk neutron and annealing effects on performance can only be evaluated in an integrated facility producing $14 \mathrm{MeV}$ fusion neutrons.

\section{SUMMARY AND PATH FORWARD}

Following the momentum initiated by the construction of the NIF laser and anticipated fusion ignition this year, we propose to construct an operational fusion power plant. The laser system for LIFE can be achieved by developing a few key technologies, and precision engineering of existing technologies to match our design requirements. One example of such a key technology is the cylindrical spatial filter, which could enhance MTBF of the beamline LRU. While these do not pose any challenges from a basic physics perspective, they require a robust engineering solution.

In the near term, small scale engineering prototypes will be realized as a basis for a design effort. These laser designs will be undertaken as part of an overall power plant design process to ensure self-consistency and system-level optimization of performance. Critical to these initial studies is an accelerated testing facility for the laser components and optics to evaluate and establish baseline lifetime values for optics. This data will be used to select materials and processes to be used with life optics and laser systems as well as guide development strategies and mitigation efforts for optics of the future. A high repetition rate laser system running at $100 \mathrm{~Hz}$ has been proposed which could generate lifetime data over 1 billion shots in a 4 month time period. ${ }^{60}$ The engineering prototypes and accelerated testing will culminate in a 
"LIFElet," a single complete beamline demonstration of a LIFE plant which includes focus into a simulated target chamber with target injection and engagement demonstration. This will serve as the final basis for the first LIFE power plant where the integration and interoperability of the system will be evaluated. Immediately following the LIFElet demonstration, construction of the first LIFE plant will begin with intended average power operations producing power.

\section{ACKNOWLEDGMENTS}

We thank Ray Beach for providing a copy of his code for predicting gain and stored energy for diode-pumped solid-state amplifiers and John Trenholme for providing detailed parameter fits from his ray-trace model for predicting ASE decay rates in laser slabs. We thank Dr. Olivier Rabot for sharing Quantel's results on diode pump reliability. This work was performed under the auspices of the U.S. Department of Energy by Lawrence Livermore National Laboratory under Contract DEAC52-07NA27344.

\section{REFERENCES}

1. C. A. HAYNAM et al., "National Ignition Facility Laser Performance Status," Appl. Opt. 46, 3276 (2007).

2. E.I. MOSES, "Ignition on the National Ignition Facility: a path towards inertial fusion energy," Nuc. Fus., 49, 104022 (2009).

3. M. DUNNE et al., "Timely Delivery Of Laser Inertial Fusion Energy (LIFE)," Fus. Sci. Tech, 2011 (in this issue).

4. J.F. LATKOWSKI et al., "Chamber Design for LIFE," Fus. Sci. Tech, 2011 (in this issue).

5. T. ANKLAM et al., "LIFE System Design," Fus. Sci. Tech, 2011 (in this issue).

6. A.J. BAYRAMIAN et al., "The Mercury Project: A High Average Power, Gas-Cooled Laser For Inertial Fusion Energy Development," Fus. Sci. \& Tech., 52, 383-387 (2007).

7. P. AMENDT et al., "LIFE Pure-Fusion Target Designs: Status and Future Prospects," Fus. Sci. Tech, 2011 (in this issue).

8. G.F. ALBRECHT et al., Appl. Opt. 29, 3079 (1990).

9. S. B. SUTTON, G. F. ALBRECHT, "Optimum performance considerations for a large aperture average power solid-state laser amplifier," J. Appl. Phys., 69, 1183 (1991).

10. P.M. CELLIERS et al., "Spatial filter pinhole for high-energy pulsed lasers," Appl. Opt., 37, 23712378 (1998).

11. J.E. MURRAY, D. MILAM, C.D. BOLEY, K.G. ESTABROOK, AND J.A. CAIRD, "Spatial filter pinhole development for the National Ignition Facility," Appl. Opt., 39, 1405-1420 (2000).

12. KURNIT et. al, SPIE 3492, 896-900, 1999.

13. A.L. BULLINGTON et al., "Thermal birefringence and depolarization compensation in glass-based highaverage-power laser systems" Proc. SPIE, 7916, 79160V-1-9, (2011).

14. E.C. HONEA, R.J. BEACH, et al., "High-power dual-rod Yb:YAG laser", Opt. Lett., 25, 805-807, (2000).

15. J. HONIG, et al., "Diode-pumped Nd:YAG laser with $38 \mathrm{~W}$ average power and user-selectable, flat-intime subnanosecond pulses," Appl. Opt., 46, 32693275, (2007).

16. C. A. EBBERS, J. HAPPE, N. NIELSEN, S. P. VELSKO, "Optical absorption at $1.06 \mathrm{~m}$ in highly deuterated potassium dihydrogen phosphate" Appl. Opt. 31 1960-1964 (1992).

17. T.I. SURATWALA, et al., "HF-Based Etching Processes for Improving Laser Damage Resistance of Fused Silica Optical Surfaces," J. Am. Ceram. Soc., accepted in publication, 1-13, (2010).

18. I.L. BASS, G.M. GUSS, M.J. NOSTRAND, AND P.J. WEGNER, "An Improved Method of Mitigating Laser Induced Surface Damage Growth in Fused Silica Using a Rastered, Pulsed $\mathrm{CO}_{2}$ Laser," Proc. SPIE, 7842, 7842201-12, (2010).

19. L. M. FRANTZ et al., J. Appl. Phys., 34, 2346-2349 (1963).

20. S. W. HANEY et al., Proc. SPIE 3047, 546 (1996).

21. R. A. SACKS et al., Proc. SPIE 3047, 526 (1996).

22. R.A. SACKS, M.A. HENESIAN, S.W. HANEY, AND J.B. TRENHOLME, "The PROP'92 Fourier beam propagation code," ICF Quarterly Report, Lawrence Livermore National Laboratory, 6, 207213, (1996).

23. P. DONNAT, C. TREIMANY, N. L'HULLIER, V. RIVOIRE, AND O. MORICE, "The Miro' software: a brief presentation,"' SPIE, 3047,102-105, (1996).

24. O. MORICE, "Miro': Complete modeling and software for pulse amplification and propagation in high-power laser systems", Opt. Eng., 42, 1530-1541 (2003).

25. J. L. EMMETT et al., "The Future of High-AveragePower Solid-State Laser," publication UCRL-53571, Lawrence Livermore National Laboratory, Livermore, CA 94550 (1984).

26. D. SCHLEUNING et al., Proc. SPIE 7198, 719802 (2009).

27. P. CRUMP et al., Photon. Technol. Lett. 20, 1378 (2008).

28. P. CRUMP et al., Proc. SPIE 6397, 639706 (2006).

29. M. KANSKAR et al, Electron. Lett. 41, 245 (2005).

30. M. KANSKAR et al, Tech. Digest $22^{\text {nd }}$ Sol. St. Diode Laser Technol. Review 41 (2005). 
31. J.-F. SEURIN et al., Proc. SPIE 6876, 68760D-1 (2008).

32. H. LI et al., Proc. SPIE 6876, 68760G-1 (2008); Photon. Technol. Lett. 19, 960 (2007).

33. D. SCHROEDER et al., Proc. SPIE 6456, 64560N-1 (2007).

34. J. A. CAIRD et al., "Nd:Glass Laser Design For Laser ICF Fission Energy (LIFE)," Fusion. Sci. Technol. 56, 607-617 (2009).

35. A. BAYRAMIAN et al., "Compact, Efficient, LowCost Diode Power Conditioning for Laser Inertial Fusion Energy," Proc. SPIE, 7916, 79160B-1-5, (2011).

36. M. MULLER et al., Proc. SPIE 6456, 64561B-1 (2007).

37. H. KISSEL et al., Proc. SPIE 6876, 687618 (2008).

38. E. DEICHSEL et al., Proc. SPIE 6876, 68760K (2008).

39. QUANTEL, Olivier Rabot, private communication based on reliability tests for the ESA's ADM-Aeolus ALADIN instrument (http://www.esa.int/esaLP/ SEMQT7ARR1F_LPadmaeolus_0.html).

40. N. LEI et al., Proc. 10th Conf. Thermal Thermomech. Phenomena Electron. Syst. (ITHERM), May 2006, p. 9, IEEE (2006).

41. S.A. PAYNE et al., "Laser properties of a new average-power Nd-doped phosphate glass," Appl. Phys. B, 61, 257-266 (1995).

42. C. P. KHATTAK, P. J. GUGGENHEIM AND F. SCHMID, "Growth of 15-Inch Diameter Sapphire Boules," Proc. SPIE, 5078, (2003).

43. J. W. LOCHER, H. E. BATES, C. D. JONES, AND S. A. ZANELLA, "Producing large EFG sapphire sheet for VIS-IR $(500-5000 \mathrm{~nm})$ window applications," Proc. SPIE, 5786, 147 (2005).

44. Sawyer Technical Products website, http://sawyerllc.com/saw.htm.

45. D. EIMERL, IEEE J. Quantum Electron. QE-23, 2238, (1987).

46. J. GOLDHAR and M.A. HENESIAN, IEEE J. Quantum Electron. QE-22, 1137 (1986).

47. W.T. PAWLEWICZ et al., Appl. Phys. Lett. 34, 196 (1979).

48. M.D. SKELDON et al., Proc. SPIE, 1410, 116 (1991).

49. K. FUCHSEL et al., Applied Optics 47, C297 (2008).

50. R.A. ZACHARIAS et al., "Alignment and wavefront control systems of the National Ignition Facility," Opt. Eng., 43, 2873-2884 (2004).

51. S.C. BURKHART et al., "National Ignition Facility system alignment," App Optics, 50, 1136 (2011).

52. P.J. WEGNER, M.A. HENESIAN, et al., "Harmonic Conversion of Large-Aperture 1.05- $\mu \mathrm{m}$ Laser Beams for Inertial-Confinement Fusion Research," Applied Optics, 31, 6414 (1992).
53. J.D. LINDL et al., "The physics basis for ignition using indirect-drive targets on the National Ignition Facility," Physics of Plasmas 11, 339 (2004).

54. D. EIMERL, "High Average Power Harmonic Generation", IEEE J. Quantum Electronics QE-23, 575 (1987).

55. D. EIMERL and D. MILAM, "Alternating-Z Tripler with High Dynamic Range," Lawrence Livermore National Laboratory Document No. UCRL-ID125409 (1996).

56. D. EIMERL, J. M. AUERBACH, C.E. BAKER, AND D. MILAM, Opt. Lett., 22, 1208-1210, (1997).

57. A. BABUSHKIN, R.S. CRAXTON, et al., "Demonstration of the dual-tripler scheme for increased-bandwidth third-harmonic generation," Opt. Lett., 23, 927-929, (1998).

58. C.D. MARSHALL, J.A. SPETH, S.A. PAYNE, "Induced optical absorption in gamma, neutron and ultraviolet irradiated fused quartz and silica," J. NonCrys. Sol,. 212, 59-73, (1997).

59. J.F. LATKOWSKI et al., "Fused Silica Final Optics for Inertial Fusion Energy: Radiation Studies and System-level Analysis," Fus. Sci. Tech., 43, 540-558, (2003).

60. A.J. BAYRAMIAN et al., "Laser Technology Test Facility for Laser Inertial Fusion Energy (LIFE)," J. Phys.: Conf. Series, 244, 032016 (2010). 


\title{
Comparison of Nd:phosphate glass, Yb:YAG and Yb:S-FAP laser beamlines for laser inertial fusion energy (LIFE) [Invited]
}

\author{
A. C. Erlandson,* S. M. Aceves, A. J. Bayramian, A. L. Bullington, R. J. Beach, \\ C. D. Boley, J. A. Caird, R. J. Deri, A. M. Dunne, D. L. Flowers, M. A. Henesian, \\ K. R. Manes, E. I. Moses, S. I. Rana, K. I. Schaffers, M. L. Spaeth, C. J. Stolz, and \\ S. J. Telford \\ Lawrence Livermore National Laboratory, PO Box 808, Livermore, CA 94551, USA \\ *erlandson1@llnl.gov
}

\begin{abstract}
We present the results of performance modeling of diodepumped solid state laser beamlines designed for use in Laser Inertial Fusion Energy (LIFE) power plants. Our modeling quantifies the efficiency increases that can be obtained by increasing peak diode power and reducing pump-pulse duration, to reduce decay losses. At the same efficiency, beamlines that use laser slabs of $\mathrm{Yb}$ :YAG or Yb:S-FAP require lower diode power than beamlines that use laser slabs of Nd:phosphate glass, since $\mathrm{Yb}: \mathrm{YAG}$ and $\mathrm{Yb}: \mathrm{S}-\mathrm{FAP}$ have longer storage lifetimes. Beamlines using Yb:YAG attain their highest efficiency at a temperature of about $200 \mathrm{~K}$. Beamlines using Nd:phosphate glass or Yb:S-FAP attain high efficiency at or near room temperature.
\end{abstract}

(C)2011 Optical Society of America

OCIS codes: (160.3380) Laser materials; (140.0140) Lasers and laser optics.

\section{References and links}

1. E. I. Moses, "The National Ignition Facility (NIF): A path to fusion energy," Energy Convers. Manage. 49(7), 1795-1802 (2008).

2. M. Dunne, E. I. Moses, P. Amendt, T. Anklam, A. Bayramian, E. Bliss, B. Debs, R. Deri, T. D. De La Rubia, B. El-Dasher, J. C. Farmer, D. Flowers, K. J. Kramer, L. Lagin, J. F. Latkowski, J. Lindl, W. Meier, R. Miles, G. A. Moses, S. Reyes, V. Roberts, R. Sawicki, M. Spaeth, and E. Storm, "Timely delivery of laser inertial fusion energy (LIFE)," Fusion Sci. Technol. 60, 19-27 (2011).

3. M. Dunne, N. Alexander, F. Amiranoff, P. Aguer, S. Atzeni, H. Azechi, V. Bagnoud, P. Balcou, J. Badziak, D. Batani, C. Bellei, D. Besnard, R. Bingham, J. Breil, M. Borghesi, S. Borneis, A. Caruso, J. C. Chanteloup, R. J. Clarke, J. L. Collier, J. R. Davies, J.-P. Dufour, P. Estraillier, R. G. Evans, M. Fajardo, R. Fedosejevs, G. Figueria, J. Fils, J. L. Feugeas, M. Galimberti, J.-C. Gauthier, A. Giulietti, L. A. Gizzi, D. Goodin, G. Gregori, S. Gus'kov, L. Hallo, C. Hernandez-Gomez, D. Hoffman, J. Honrubia, S. Jacquemot, M. Key, J. Kilkenny, R. Kingham, M. Koenig, F. Kovacs, K. Krushelnic, C. Labaune, K. Lancaster, C. Leblanc, P. H. Maire, W. Martin, A. McEvoy, P. McKenna, J. T. Mendonça, J. Meyer-ter-Vehn, K. Mima, G. Mourou, S. Moustaizis, Z. Najmudin, P. Nickles, D. Neely, P. Norreys, M. Olazabal, A. Offenberger, N. Papadogianis, J.-P. Perin, J. M. Perlado, J. Ramirez, R. Ramis, Y. Rhee, X. Ribeyre, A. Robinson, K. Rohlena, S. J. Rose, M. Roth, C. Rouyer, C. Rulliere, B. Rus, W. Sandner, A. Schiavi, G. Schurtz, A. Sergeev, M. Sherlock, L. Silva, R. A. Smith, G. Sorasio, C. Strangio, H. Takabe, M. Tatarakis, V. Tikhonchuk, M. Tolley, M. Vaselli, P. Velarde, T. Winstone, K. Witte, J. Wolowski, N. Woolsey, B. Wyborn, M. Zepf, and J. Zhang, "HiPER - technical background and conceptual design report" (HiPER Project, 2011). http://www.hiper-laser.org/docs/tdr/HiPERTDR2.pdf.

4. T. Norimatsu, Y. Kozaki, N. Miyanaga, J. Kawanaka, H. Azechi, T. Johzaki, and K. Tomabechi, "Conceptual design of laser fusion reactor KOYO-F based on fast ignition scheme," in Proceedings of the 21st IAEA Conference (International Atomic Energy Agency, 2008), paper FT/P5-39. http://wwwpub.iaea.org/MTCD/Meetings/FEC2006/ft_p5-39.pdf.

5. J. Kawanaka, N. Miyanaga, T. Kawashima, K. Tsubakimoto, Y. Fujimoto, H. Kubomura, S. Matsuoka, T. Ikegawa, Y. Suzuki, N. Tsuchiya, T. Jitsuno, H. Furukawa, T. Kanabe, H. Fujita, K. Yoshida, H. Nakano, J. Nishimae, M. Nakatsuka, K. Ueda, and K. Tomabechi, "New concept for laser fusion energy driver by using cryogenically-cooled Yb:YAG ceramic," J. Phys.: Conf. Ser. 112, (2008), doi:10.1088/1742-6596/112/3/032058.

6. C. D. Orth, S. A. Payne, and W. F. Krupke, "A diode pumped solid state laser driver for inertial fusion energy," Nucl. Fusion 36(1), 75-116 (1996). 
7. H. Matsui, T. Eguchi, T. Kanabe, M. Yamanaka, M. Nakatsuka, Y. Izawa, and S. Nakai, "Conceptual design of a laser diode-pumped Nd:glass slab laser driver for inertial fusion energy,” Fusion Eng. Des. 44(1-4), 401-405 (1999).

8. T. M. Anklam, A. M. Dunne, W. R. Meier, S. Powers, and A. J. Simon, "LIFE: the case for early commercialization of fusion energy," Fusion Sci. Technol. 60, 66-71 (2011).

9. J. F. Latkowski, R. P. Abbott, S. Aceves, T. M. Anklam, A. W. Cook, J. DeMuth, L. Divol, B. El-Dasher, J. C. Farmer, D. Flowers, M. Fratoni, T. Heltemes, J. Kane, K. J. Kramer, R. Kramer, A. Lafuente, G. A. Loosmore, K. R. Morris, G. A. Moses, B. Olson, C. Pantano, S. Reyes, M. Rhodes, R. Sawicki, H. Scott, M. Tabak, and S. Wilks, "Chamber design for LIFE," Fusion Sci.Technol. 60, 54-60 (2011).

10. A. Bayramian, S. Aceves, T. Anklam, K. Baker, E. Bliss, C. Boley, A. Bullington, J. Caird, D. Chen, R. Deri, M. Dunne, A. Erlandson, D. Flowers, M. Henesian, J. Latkowski, K. Manes, W. Molander, E. Moses, T. Piggott, S. Powers, S. Rana, S. Rodriguez, R. Sawicki, K. Schaffers, L. Seppala, M. Spaeth, S. Sutton, and S. Telford, "Compact, efficient laser systems required for laser inertial fusion energy," Fusion Sci. Technol. 60, 28-48 (2011).

11. Data sheet for APG-1 laser glass, (Schott North America, Inc., 2011). http://www.schott.com/advanced_optics/english/download/catalogs.html.

12. J. H. Campbell, J. S. Hayden, and A. Marker, "High-power solid-state lasers: a laser glass perspective," Int. J. Appl. Glass Sci. 2(1), 3-29 (2011).

13. L. D. DeLoach, S. A. Payne, L. K. Smith, W. L. Kway, and W. F. Krupke, "Laser and spectroscopic properties of $\mathrm{Sr}_{5}\left(\mathrm{PO}_{4}\right)_{3} \mathrm{~F}: \mathrm{Yb}, ” J$ J. Opt. Soc. Am. B 11(2), 269-276 (1994).

14. A. J. Bayramian, "Development of trivalent ytterbium doped fluorapatites for diode-pumped laser applications," doctoral thesis, University of California at Davis, UCRL-LR-139215, (June 21, 2000).

15. A. Ikesue, T. Kinoshita, K. Kamata, and K. Yoshida, "Fabrication and optical properties of high-performance polycrystalline Nd:YAG ceramics for solid-state lasers," J. Am. Ceram. Soc. 78(4), 1033-1040 (1995).

16. A. Ikesue, Y. L. Aung, T. Yoda, S. Nakayama, and T. Kamimura, "Fabrication and laser performance of polycrystal and single crystal Nd:YAG by advanced ceramic processing," Opt. Mater. 29(10), 1289-1294 (2007).

17. S. A. Payne, L. D. DeLoach, L. K. Smith, W. L. Kway, J. B. Tassano, W. F. Krupke, B. H. T. Chai, and G. Loutts, "Ytterbium-doped apatite-structure crystals: a new class of laser materials," J. Appl. Phys. 76(1), 497503 (1994).

18. K. Schaffers, A. J. Bayramian, J. A. Menapace, G. T. Rogowski, T. F. Soules, C. A. Stolz, S. B. Sutton, J. B. Tassano, P. A. Thelin, C. A. Ebbers, J. A. Caird, C. P. J. Barty, M. A. Randies, C. Porter, Y. Fei, and B. H. T. Chai, "Advanced material development for inertial fusion energy (IFE)," in Crystal Growth Technology, P. Capper and P. Rudolph, eds. (Wiley-VCH Verlag, 2010).

19. K. I. Schaffers, J. B. Tassano, A. B. Bayramian, and R. C. Morris, "Growth of Yb: S-FAP $\left[\mathrm{Yb}^{3+:} \mathrm{Sr}_{5}\left(\mathrm{PO}_{4}\right)_{3} \mathrm{~F}\right]$ crystals for the mercury laser," J. Cryst. Growth 253(1-4), 297-306 (2003), doi: 10.1016/S0022-0248(03)010327, http://apex.jsap.jp/link?APEX/4/022703/.

20. J. Akiyama, Y. Sato, and T. Taira, "Laser demonstration of diode-pumped $\mathrm{Nd}^{3+}$-doped fluorapatite anisotropic ceramics," Appl. Phys. Express 4(2), 022703 (2011).

21. J. T. Hunt, J. A. Glaze, W. W. Simmons, and P. A. Renard, "Suppression of self-focusing through low-pass spatial filtering and relay imaging," Appl. Opt. 17(13), 2053-2057 (1978).

22. C. A. Haynam, P. J. Wegner, J. M. Auerbach, M. W. Bowers, S. N. Dixit, G. V. Erbert, G. M. Heestand, M. A. Henesian, M. R. Hermann, K. S. Jancaitis, K. R. Manes, C. D. Marshall, N. C. Mehta, J. Menapace, E. Moses, J. R. Murray, M. C. Nostrand, C. D. Orth, R. Patterson, R. A. Sacks, M. J. Shaw, M. Spaeth, S. B. Sutton, W. H Williams, C. C. Widmayer, R. K. White, S. T. Yang, and B. M. Van Wonterghem, "National Ignition Facility laser performance status," Appl. Opt. 46(16), 3276-3303 (2007).

23. X. Julien, A. Adolf, E. Bar, V. Beau, E. Bordenave, T. Chiès, R. Courchinoux, J. M. Di-Nicola, C. Féral, P. Gendeau, H. Graillot, C. Grosset-Grange, O. Henry, V. Higonenq, E. Journot, L. Lacampagne, E. Lafond, L. Le Déroff, A. Martinez, L. Patissou, A. Roques, L. Thauvin, and G. Thiell, "LIL laser performance status," Proc. SPIE 7916, 791610 (2011).

24. A. Bayramian, P. Armstrong, E. Ault, R. Beach, C. Bibeau, J. Caird, R. Campbell, B. Chai, J. Dawson, C. Ebbers, A. Erlandson, Y. Fei, B. Freitas, R. Kent, Z. Liao, T. Ladran, J. Menapace, B. Molander, S. Payne, N. Peterson, M. Randles, K. Schaffers, S. Sutton, J. Tassano, S. Telford, and E. Utterback, "The Mercury Project: A high average power, gas-cooled laser for inertial fusion energy development," Fusion Sci. Technol. 52, 383-387 (2007).

25. A. Bayramian, B. Deri, S. Fulkerson, R. Lanning, and S. Telford, "Compact, efficient, low-cost diode power conditioning for laser inertial fusion energy," Proc. SPIE 7916, 79160B (2011).

26. R. Feeler, J. Junghans, and E. Stephens, "Low-cost diode arrays for the LIFE project," Proc. SPIE 7916, 791608 (2011).

27. P. Crump, H. Wenzel, G. Erbert, P. Ressel, M. Zorn, F. Bugge, S. Einfeldt, R. Staske, U. Zeimer, A. Pietrzak, and G. Trankle, "Passively cooled TM polarized $808-\mathrm{nm}$ laser bars with $70 \%$ power conversion at 80-W and 55W peak power per 100- $\mu \mathrm{m}$ stripe width," IEEE Photon. Technol. Lett. 20(16), 1378-1380 (2008).

28. P. Crump, S. Patterson, J. Wang, W. Dong, M. Grimshaw, S. Zhang, S. Elim, M. Bougher, J. Patterson, S. Das, D. Wise, M. DeFranza, J. Bell, J. Farmer, M. DeVito, and R. Martinsen, "Diode laser bars deliver $>400$-W peak CW power from 800-nm to 980-nm,” Proc. SPIE 6397, 639706 (2006).

29. M. Kanskar, T. Earles, T. J. Goodnough, E. Stiers, D. Botez, and L. J. Mawst, "73\% CW power conversion efficiency at 50W from 970nm diode laser bars,” Electron. Lett. 41(5), 245-247 (2005).

\#151989 - \$15.00 USD Received 29 Jul 2011; revised 10 Oct 2011; accepted 15 Oct 2011; published 26 Oct 2011

(C) 2011 OSA 1 November $2011 /$ Vol. 1, No. 7 / OPTICAL MATERIALS EXPRESS 1342 
30. P. Crump, J. Wang, S. Patterson, D. Wise, A. Basauri, M. DeFranza, S. Elim, W. Dong, S. Zhang, M. Bougher, J. Patterson, S. Das, M. Grimshaw, J. Farmer, M. DeVito, and R. Martinsen, "Diode laser efficiency increases enable > 400-W peak power from 1-cm bars," Proc. SPIE 6104, 610409 (2006).

31. Temperature-dependent absorption spectra of Yb:YAG were provided by J. Kawanaka of the Institute of Laser Engineering, Osaka University, Osaka, Japan.

32. J. Dong, M. Bass, Y. Mao, P. Deng, and F. Gan, "Dependence of the Yb3+ emission cross section and lifetime on temperature and concentration in yttrium aluminum garnet," J. Opt. Soc. Am. B 20(9), 1975-1979 (2003).

33. L. M. Frantz and J. S. Nodvik, "Theory of pulse propagation in a laser amplifier," J. Appl. Phys. 34(8), 23462349 (1963).

34. A. E. Siegman, Lasers (University Science Books, 1986), Chap. 8

35. R. A. Sacks, M. A. Henesian, S. W. Haney, and J. B. Trenholme, "The PROP92 Fourier beam propagation code," in ICF Annual Report, UCRL-LR-105821-96 (Lawrence Livermore National Laboratory, Livermore, CA, 1996), pp. 207-213.

36. O. Morice, "Miro: Complete modeling and software for pulse amplification and propagation in high-power laser systems," Opt. Eng. 42(6), 1530-1541 (2003).

37. W. M. Kays and A. L. London, Compact Heat Exchangers, 3rd ed. (Krieger Publishing, 1998).

38. K. A. Manske, D. T. Reindl, and S. A. Klein, "Evaporative condenser control in industrial refrigeration systems," Int. J. Refrig. 24, 676-691 (2001).

39. J. L. Emmett, W. F. Krupke, and W. R. Sooy, The Potential of High-Average-Power Solid-State Lasers, UCRL53571 (Lawrence Livermore National Laboratory, Livermore, CA, 1984).

40. A. C. Erlandson, G. F. Albrecht, and S. E. Stokowski, "Model predicting the temperature dependence of the gain coefficient and the extractable stored energy density in Nd:phosphate glass lasers," J. Opt. Soc. Am. B 9(2), 214222 (1992).

41. J. Dong, M. Bass, and C. Walters, "Temperature-dependent stimulated-emission cross section and concentration quenching in Nd3+-doped phosphate glasses," J. Opt. Soc. Am. B 21(2), 454-457 (2004).

42. D. M. Pennington, D. Milam, and D. Eimerl, "Gain saturation studies in LG-750 and LG-770 amplifier glass," Proc. SPIE 3047, 630-642 (1997).

43. W. E. Martin, D. Milam, and J. B. Trenholme, Laser Program Annual Report-79, UCRL-50021-79, (Lawrence Livermore National Laboratory, Livermore, CA, 1980), pp. 2.160-2.170.

44. W. E. Martin and D. Milam, "Direct measurement of gain recovery in a saturated Nd-glass amplifier," Appl. Phys. Lett. 32(12), 816-818 (1978)

45. W. E. Martin and D. Milam, "Gain saturation in Nd:doped laser materials," IEEE J. Quantum Electron. 18(7), 1155-1163 (1982).

46. D. C. Brown, “The promise of cryogenic solid-state lasers,” IEEE J. Sel. Top. Quantum Electron. 11(3), 587-599 (2005).

47. T. Y. Fan, D. J. Ripin, R. L. Aggarwal, J. R. Ochoa, B. Chann, M. Tilleman, and J. Spitzberg, "Cryogenic Yb3+doped solid-state lasers," IEEE J. Sel. Top. Quantum Electron. 13(3), 448-459 (2007).

48. Y. Sato, J. Akiyama, and T. Taira, "Effects of rare-earth doping on thermal conductivity in $\mathrm{Y}_{3} \mathrm{Al}_{5} \mathrm{O}_{12} \mathrm{crystals}$," Opt. Mater. 31(5), 720-724 (2009).

\section{Introduction}

Interest in the development of laser fusion energy has been increasing in recent years, for several reasons. Demand for electrical power, particularly power that is carbon free, has been increasing. The National Ignition Facility (NIF), which uses a solid-state laser driver, is soon expected to achieve fusion ignition and gain, thus demonstrating the feasibility of the basic process laser fusion power plants would use to generate electricity. Additionally, a sea change is occurring in the cost, availability and performance of diode lasers, which enable solid-state lasers to operate at the high efficiency and repetition rate required for fusion power plants. Given these trends, it is not surprising that several groups have proposed to develop laser fusion energy based on solid-state laser drivers [1-7].

A fusion power plant concept called Laser Inertial Fusion Energy (LIFE) has recently been put forward by engineers and scientists at the Lawrence Livermore National Laboratory (LLNL) [2,8-10]. As currently envisioned, each LIFE power plant would have net electrical power generating capacity of $1 \mathrm{GW}$ and use a $2.2 \mathrm{MJ}$, 384-beam, harmonically-converted $\mathrm{Nd}$ :glass laser to ignite cryogenically-cooled, indirect-drive fusion targets. Predicted overall laser efficiency, including power drawn by the laser cooling system, is greater than $15 \%$. Details of the LIFE laser beamline design have been described before [10].

Nd-doped APG-1, a phosphate glass, was the gain medium chosen for the LIFE laser design because its properties meet requirements for efficient, high-average-power operation $[11,12]$. Specifically, storage lifetime is sufficient for pumping to be achieved using numbers of diodes that are affordable, when diodes are purchased in power-plant quantities. Saturation fluence is high enough for energy to be stored efficiently at high density, yet low enough for

\#151989 - \$15.00 USD Received 29 Jul 2011; revised 10 Oct 2011; accepted 15 Oct 2011; published 26 Oct 2011

(C) 2011 OSA

1 November 2011 / Vol. 1, No. 7 / OPTICAL MATERIALS EXPRESS 1343 
energy to be extracted efficiently at fluences below damage thresholds. Thermal shock resistance is sufficient to sustain laser operation at the required repetition rate of $16 \mathrm{~Hz}$. Additionally, risks associated with the availability of Nd-doped phosphate glass slabs, in the quality and quantities needed for fusion power-plant lasers, is small relative to availability risks for most other gain-media. Over 110 metric tons of high-quality phosphate laser glass, distributed over 3072 slabs with dimensions of $4 \mathrm{~cm} \mathrm{x} 44 \mathrm{~cm} \times 74 \mathrm{~cm}$, has already been fabricated for the NIF laser at LLNL. A similar quantity has been fabricated for the Laser Megajoule in Ceste, France. In comparison, the LIFE laser design uses only 27 metric tons of phosphate laser glass, which is distributed over 15,360 laser slabs with dimensions of $1 \mathrm{~cm} \mathrm{x}$ $26 \mathrm{~cm} \times 26 \mathrm{~cm}$. The LIFE laser uses less phosphate glass than the NIF laser because stored energy density in the slabs is higher, and because less energy needs to be stored due to higher laser efficiency.

We have considered two other candidate gain media for use in LIFE laser beamline designs: Yb-doped yttrium aluminum garnet (Yb:YAG) and $\mathrm{Yb}$-doped strontium fluorapatite (Yb:S-FAP). Both materials have been considered previously for use in fusion power plants and have potential performance advantages relative to Nd-doped phosphate glass [3-6]. With their longer storage lifetimes, Yb:YAG and Yb:S-FAP require fewer diodes for pumping. Since laser pump diodes are a major cost center for solid-state fusion lasers, this is an especially important consideration. Another advantage, which arises from the relatively simple, two-state electronic structure of $\mathrm{Yb}^{3+}$ ions, is immunity from several loss mechanisms that affect $\mathrm{Nd}^{3+}$ and other rare-earth lasers, including excited-state absorption, concentration quenching and upconversion. YAG also has much higher thermal conductivity and higher thermal shock resistance than phosphate glass. A disadvantage of Yb:YAG, however, is the necessity to cool slabs to temperatures well below room temperature for efficient operation. Cooling is required to reduce the thermal population of the lower laser level, which absorbs a portion of the extracting laser beam. Cooling also reduces the saturation fluence, so that stored energy can be extracted more efficiently without causing optical damage. Yb:S-FAP has more fortuitous spectral features than Yb:YAG that enable it to operate efficiently even at room temperature $[13,14]$.

To date, Yb:YAG and Yb:S-FAP have been manufactured only in much smaller quantities and slab sizes than would be required for a LIFE power plant. However, excellent progress on the development of fabrication technology for these and similar materials has occurred. Fabrication of transparent ceramic YAG material using vacuum sintering is particularly salient $[15,16]$, as this process appears readily scalable to large slab size. As the time required for fabrication is quite small, the vacuum sintering method appears amenable to mass production. Advances in technique have reduced scattering losses to $\sim 0.1 \% / \mathrm{cm}$.

Progress has also been reported on the development of Yb:S-FAP crystals. Growth of the first small, high-quality crystal boules were reported in 1994 [17]. Subsequently, a program was conducted, both at LLNL and at Northrup Grumman, to develop larger crystals for use in the Mercury laser system, at LLNL [18]. A number of crystal growth issues were discovered and addressed, but the issue of the occurrence of growth defects that cause small-scale wavefront features still remains. Nonetheless, crystals of sufficient size $(0.7 \mathrm{~cm} \mathrm{x} 4 \mathrm{~cm} \times 6$ $\mathrm{cm}$ ) and quality were fabricated for use in the Mercury laser, which produced 60-J pulses at $10-\mathrm{Hz}$ [19]. A recent development is the fabrication of C-FAP transparent ceramic [20]. CFAP has the same crystalline structure as S-FAP but with calcium ions replacing strontium ions. Since C-FAP, like S-FAP, is uniaxial, it was necessary to align the constituent nanocrystals, to manage scattering losses. Alignment was achieved using a strong magnetic field. If successfully developed, this method has the potential for manufacturing laser slabs of the size needed for LIFE lasers, while avoiding the defect issues that attend Yb:S-FAP crystal growth.

In this paper, we examine and compare the predicted performance of laser beamlines that use laser slabs of Nd:APG-1, Yb:YAG and Yb:S-FAP. A goal of our work is to provide materials developers with a measure of the relative performance of these three different gain media, when they are used in a laser architecture that enables high efficiencies to be attained.

\#151989 - \$15.00 USD Received 29 Jul 2011; revised 10 Oct 2011; accepted 15 Oct 2011; published 26 Oct 2011

(C) 2011 OSA 1 November 2011 / Vol. 1, No. 7 / OPTICAL MATERIALS EXPRESS 1344 
We have attempted to make these calculations comprehensive by including all laser energy transfer processes as well as the electrical power consumed by the cooling system. The remaining sections of this paper are as follows. In Section 2, we describe the LIFE laser beamline design. In Section 3, we describe our model for calculating efficiency. In Section 4, we describe calculations performed using our model. We present our modeling results in Section 5 and we provide a summary in Section 6.

\section{Laser design}

Figure 1 provides a schematic diagram of the LIFE laser beamline design [10]. Following established practices for high-energy pulsed lasers such as the NIF, the LIFE laser design uses far-field spatial filtering to control the nonlinear growth of small-scale intensity features and image relaying to limit the negative impacts of diffraction on beam quality [21]. Also, like the NIF and LMJ lasers, the laser pulse is injected into the main amplifier cavity by reflecting a low-energy seed beam from a small mirror near the far field of a telescope [22,23]. The beam is collimated, at the desired size, by one of the telescope lenses. To ensure high extraction efficiency, the laser beam has high fill factor and, after four passes, exits the amplifier cavity at a fluence that is several times the saturation fluence. A quarter-wave plate located between the main amplifiers and the polarizer causes the beam to be circularly polarized as it propagates through the amplifiers and to be linearly polarized when it is incident on the polarizer. Circularly-polarized light has one-third less nonlinear phase shift than linearlypolarized light and reduced risk of beam break up into high-spatial-frequency intensity features. As the two amplifiers are designed to be pumped and cooled similarly and to have matching temperature distributions, the $90^{\circ}$ quartz polarization rotator placed between the two amplifiers causes the thermal birefringence of one amplifier to offset the thermal birefringence of the other. The quartz polarization rotator also causes the beam to be spolarized and to reflect off the polarizer after four passes through the amplifiers, without need to use an active polarization switch. The large Pockels cell, which is not used for switching, is used to protect the front end from back-reflected pulses and to reduce risk for parasitic lasing.

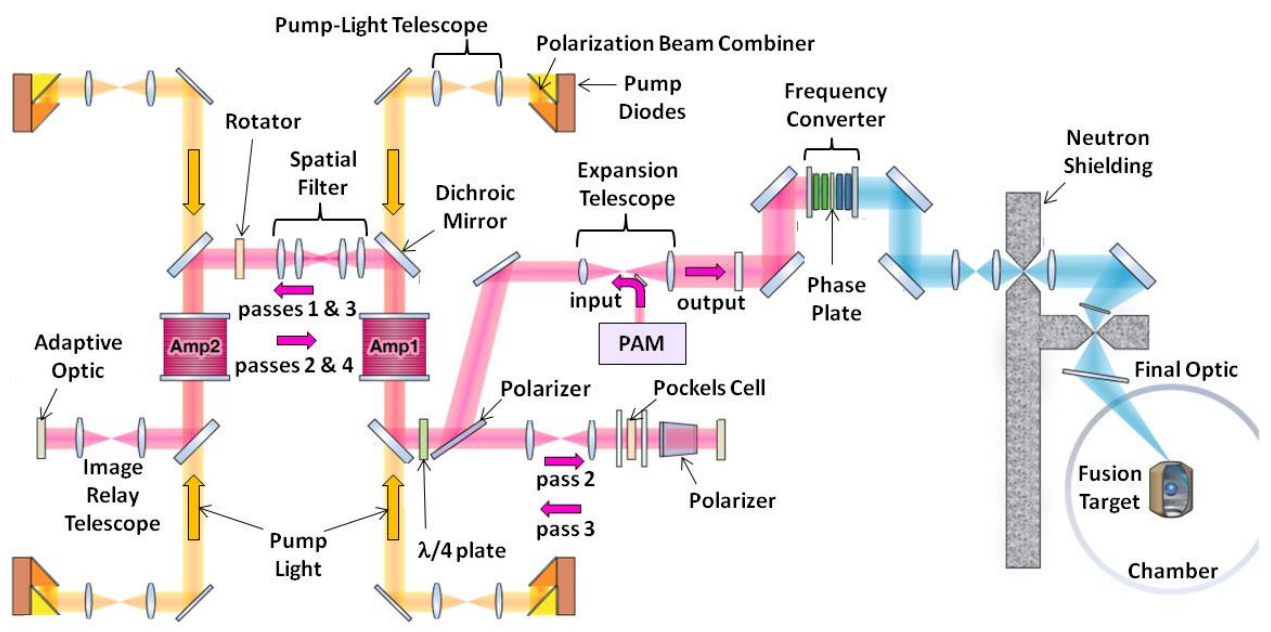

Fig. 1. Schematic diagram showing the LIFE laser beamline design, which was used for our efficiency evaluations of Nd-doped phosphate glass, Yb-doped YAG, and Yb-doped S-FAP slabs. For Yb:S-FAP, the quarter-wave plate was removed and the rotator was moved to a location near the adaptive optic, to accommodate Yb:S-FAP's natural birefringence.

Since requirements for the LIFE laser's efficiency and repetition rate are more demanding $(>12 \%$ and $16 \mathrm{~Hz}$, respectively) than performance characteristics of the NIF laser $(\sim 1 \%$ efficiency and $\sim 10^{-4} \mathrm{~Hz}$, respectively), the LIFE laser design is different from the NIF laser 
design in several respects. While the NIF laser slabs are pumped by flashlamps, the LIFE laser slabs are pumped by laser diodes. Diode laser light is more directional and more spectrally narrow than flashlamp light, and is transported to the laser slabs and is absorbed more efficiently. Diode light also reduces the quantum defect (energy difference between pump photons and extracting laser photons), particularly when pumping within the red-most pump band of $\mathrm{Nd}^{3+}$ at $\sim 873 \mathrm{~nm}$, as is the case for the LIFE laser. Further, while the NIF laser slabs are passively cooled, the LIFE laser slabs are actively cooled by forced convection of helium gas over slab surfaces. The technological basis for pumping with diodes and cooling with helium gas is well established and has been used before in the Mercury laser at LLNL [24].

Amplifiers comprise a stack of face-pumped Nd:APG-1 glass laser slabs, oriented at near normal incidence to the laser beam. APG-1 is a phosphate glass that has high thermal shock resistance relative to most other phosphate laser glasses. Slab thickness is one $\mathrm{cm}$, thin enough to ensure that tensile stresses at the large slab surfaces are less than $\sim 25 \%$ of the yield stress. Slabs are separated by two-mm-thick cooling channels in which helium gas is flowed to remove waste heat. Cooling channels for the outward-facing surfaces of the outer two slabs are defined by fused-silica windows, which are sufficiently thick $(\sim 7 \mathrm{~cm})$ to hold off the difference between the helium cooling gas pressure and the outside ambient pressure $(\sim 4$ times atmospheric pressure). A high helium gas pressure reduces power consumption by the helium compressor, under the constraint that the helium mass-flow rate is sufficient to limit the caloric temperature rise of the gas as it flows over the slabs, to $10 \mathrm{~K}$. Slabs are edge mounted using a low-modulus transparent material that transmits amplified spontaneous emission from the slab to a liquid-cooled absorbing edge cladding. All transmissive optics have anti-reflective coatings.

Laser slab stacks are pumped on each end by pump-light modules, which produce intense, high-fill-factor, flat-top pump distributions. Diode light originates in closely-packed, highpower arrays of diode bars, which use microlenses to achieve low divergence $\left(4^{\circ}\right.$ fast axis $\mathrm{x}$ $10^{\circ}$ slow axis). On each amplifier end, light from two different arrays are combined using a polarizer, which nearly doubles the irradiance available from a single array. A half-wave plate is placed in front of one array to implement this polarization-combining method. A telescope images light from the two diode arrays onto the slab stack. Diode arrays are offset parallel to their emitting surface, to blur imprinting of individual diode tile structures onto the pump profile.

We used the beamline design, as shown in Fig. 1, to model performance with Nd:APG-1 slabs and Yb:YAG slabs. Both gain media are anisotropic and transmit circularly polarized light without difficulty. When modeling performance with Yb:S-FAP, which is uniaxial, the quarter-wave plate, Q1, was removed so that the beam propagating through the slabs was linearly polarized. The beam is polarized in the direction of the high-gain extraordinary axis on the first and fourth passes through the system, and in the direction of low-gain, ordinary axis on the second and third passes.

\section{Efficiency model}

We performed detailed efficiency calculations in which all energy transfer processes were taken into account, beginning with input electrical power and ending with optical power delivered to the final optic, which focuses light onto the target. See Table 1. The efficiency for conversion of alternating current to direct current (AC-DC) reflects the current state of commercial rectification technologies. The efficiency for the electrical pulser reflects results of recent prototype pulser tests, which have demonstrated $90 \%$ efficiency. However, circuit simulations show that anticipated improvements in the series resistance of capacitors and other components will increase pulser efficiency to 95\% [25]. The efficiency for diodes reflects industry consensus on diode performance that will be achieved with mass-produced, low-cost diodes within a few years, and on efficiencies reported in the literature for diode lasers operating at $808 \mathrm{~nm}$ and 940-970 nm [e.g., 26-29] and at 850-980 nm [30], which have exceeded $70 \%$. Pump light delivery and absorption efficiencies were calculated using raytrace simulations of the pump delivery system described above, and measured absorption

\#151989 - \$15.00 USD Received 29 Jul 2011; revised 10 Oct 2011; accepted 15 Oct 2011; published 26 Oct 2011

(C) 2011 OSA 1 November 2011 / Vol. 1, No. 7 / OPTICAL MATERIALS EXPRESS 1346 
spectra for Nd-doped APG-1 glass [11], Yb:YAG [31] and Yb:S-FAP [14]. Diode emission spectra were calculated assuming an instantaneous Gaussian distribution with a full-width, half-maximum bandwidth of $5 \mathrm{~nm}$, and a time-dependent spectral chirp due to rising diode temperature during the pump pulse. Overall decay rates for excited ions were found by summing decay rates for all known significant decay channels, including spontaneous radiative decay rates [11,13,32], concentration quenching rates (for Nd:APG-1 only) [11], and amplified spontaneous emission rates. Amplified spontaneous emission (ASE) and radiation trapping rates were calculated using Monte-Carlo ray-tracing techniques that include both spectral and geometric effects, including transfer of radiation from slab to slab within the slab stack.

Extraction efficiency was calculated using the Frantz-Nodvik formalism [33,34], which was applied to individual slabs and with transmission losses taken into account at the input and output surfaces of each slab. To model pulse shape and nonlinear phase shift of the beam as it propagates through the beamline, laser pulses were divided into over one-hundred time slices. An iterative routine was used to adjust the input pulse shape and energy to achieve the desired output pulse. The extraction efficiency reported in Table 1 is the extracted fluence at the centerline of the laser aperture, divided by the centerline stored fluence. Stored fluence was calculated by multiplying the gain in nepers by the saturation fluence. The effect on efficiency of imperfect overlap between the gain distribution and the extracting beam was captured by the mode overlap factor. Mode overlap factor and the impact of gain nonuniformity were calculated using simulation codes [35,36] that have been extensively validated against high-energy pulse laser systems, and which include spatial variations across the beam profile.

The slab pumping simulations incorporated heating and cooling effects. Heat sources included waste heat generated by the diodes, waste heat generated by pump processes and thermalized within the laser slabs, and absorption of spontaneous emission and amplified spontaneous emission by edge claddings surrounding each slab. Our simulations used standard techniques $[37,38]$ to calculate power consumption of chillers, compressors, pumps, and heat exchangers, as required for cooling diodes, slabs and edge claddings. Coolant pump power was calculated using pressure drops for specific cooling-channel dimensions and coolant mass-flow rates needed to limit temperature rise along flow paths. The caloric temperature rise of helium gas flowing over the laser slabs was limited to $10 \mathrm{~K}$. The caloric rise of liquid coolant flowing over the edge claddings was limited to $20 \mathrm{~K}$. Slab temperature distributions and tensile stresses at the large faces were approximated using standard formulas for uniform heat deposition and cooled faces [39].

\#151989 - \$15.00 USD Received 29 Jul 2011; revised 10 Oct 2011; accepted 15 Oct 2011; published 26 Oct 2011

(C) 2011 OSA 1 November 2011 / Vol. 1, No. 7 / OPTICAL MATERIALS EXPRESS 1347 
Table 1. Efficiency Factors for Beamlines Using Slabs of Nd:APG-1 Phosphate Glass at $326 \mathrm{~K}$, Yb:YAG at $200 \mathrm{~K}$, and Yb:S-FAP at $295 \mathrm{~K}$

\begin{tabular}{lccc}
\multicolumn{1}{c}{ Efficiency Factor } & $\begin{array}{c}\text { Nd:APG-1 } \\
\text { @ 326 K }\end{array}$ & $\begin{array}{c}\text { Yb:YAG } \\
\text { @ 200 K }\end{array}$ & $\begin{array}{c}\text { Yb:S-FAP } \\
\text { @295K }\end{array}$ \\
\hline DC Power Supply & 95 & 95 & 95 \\
Electrical Pulsers & 95 & 95 & 95 \\
Diodes & 72 & 72 & 72 \\
Diode Micro-Lenses & 98 & 98 & 98 \\
Pump-Light Delivery System & 93 & 93 & 93 \\
Pump-Light Absorption & 99 & 92 & 92 \\
Quantum Defect & 83 & 91 & 86 \\
Spontaneous Emission, Trapping & 85 & 90 & 86 \\
Amplified Spontaneous Emission & 89 & 93 & 94 \\
Transparency & 100 & 88 & 87 \\
Energy Extraction & 81 & 95 & 95 \\
Pump-Light Non-Uniformity & 99 & 99 & 99 \\
Mode-Match & 92 & 92 & 92 \\
Infrared transport & 92 & 93 & 93 \\
Depolarization & 99 & 99 & 99 \\
Frequency Conversion & 75 & 75 & 75 \\
351 nm transport & 95 & 95 & 95 \\
\hline Electrical-to-Optical & 18 & 21 & 19 \\
Efficiency, without Cooling & 87 & 74 & 85 \\
Cooling Efficiency & 16 & 15 & 16 \\
\hline Electrical-to-Optical Efficiency, & & & \\
with Cooling & & 216 & \\
\hline
\end{tabular}

\section{Calculations}

Using the model described above, energetics calculations were performed iteratively to determine combinations of design parameters that meet laser energy and power requirements while producing high overall efficiency. The beamline designs we analyzed use the same $1 \omega$ beam size $(25 \mathrm{~cm} \times 25 \mathrm{~cm}$, including apodized borders) and the same values for efficiencies that are independent of gain medium, such as harmonic conversion efficiency and $3 \mathrm{w}$ beam transport efficiency. Square pulse distortion, the ratio of small-signal gain at the beginning of the pulse to small-signal gain at the end of the pulse, was limited to $\sim 30-40$, to ensure that output pulse shapes can be controlled accurately, given limitations on the dynamic range of pulse-shaping hardware in the laser front end. In turn, the limit on square pulse distortion gave limits on $1 \omega$ output fluences, which were different for the three different gain media due to 
Table 2. Key Properties Used for Modeling Performance of APG-1, Yb:YAG and Yb:SFAP

\begin{tabular}{|c|c|c|c|c|}
\hline & & APG-1 $^{\text {a }}$ & $\mathbf{Y b}: \mathbf{Y A G}^{\mathrm{b}}$ & Yb:S-FAP ${ }^{c}$ \\
\hline Property & Symbol & @ $326 \mathrm{~K}$ & $200 \mathrm{~K}$ & $295 \mathrm{~K}$ \\
\hline \multicolumn{5}{|l|}{ Laser Properties } \\
\hline Gain peak $(\mathrm{nm})$ & $\lambda_{\mathrm{L}}$ & 1054 & 1030 & 1047 \\
\hline Emission cross section at $\lambda_{\mathrm{L}}\left(10^{-20} \mathrm{~cm}^{2}\right)$ & $\sigma_{\mathrm{em}, \lambda}$ & 3.3 & 4.4 & 6.1 \\
\hline Absorption cross section at $\lambda_{\mathrm{L}}\left(10^{-20} \mathrm{~cm}^{2}\right)$ & $\sigma_{\mathrm{abs}, \lambda}$ & 1.4 & 0.060 & 0.320 \\
\hline Saturation fluence $\left(\mathrm{J} / \mathrm{cm}^{2}\right)$ & $\phi_{\mathrm{sat}}$ & 5.8 & 4.3 & 3.0 \\
\hline $\begin{array}{l}\text { Radiative Lifetime } \\
\text { (zero concentration limit) }(\mu \mathrm{s})\end{array}$ & $\tau_{0}$ & 385 & 995 & 1,100 \\
\hline Judd-Ofelt radiative lifetime $(\mu \mathrm{s})$ & $\tau_{\mathrm{r}}$ & 361 & - & - \\
\hline Emission band width (FWHM) (nm) & $\Delta \lambda_{\text {eff }}$ & 27.8 & 2.4 & 4.0 \\
\hline Concentration quenching factor $\left(\mathrm{cm}^{-3}\right)^{*}$ & $\mathrm{Q}$ & 16.7 & - & - \\
\hline Pump wavelength (nm) & $\lambda_{\mathrm{P}}$ & 874 & 932 & 899 \\
\hline Quantum Defect & $\eta_{\mathrm{QD}}$ & 0.829 & 0.905 & 0.859 \\
\hline \multicolumn{5}{|l|}{ Optical Properties } \\
\hline Refractive index at $\lambda_{\mathrm{L}}$ & $\mathrm{n}_{\mathrm{L}}$ & 1.526 & 1.82 & 1.612 \\
\hline Nonlinear index parameter $\left(10^{-7} \mathrm{~cm}^{2} / \mathrm{GW}\right)$ & $\gamma$ & 3.11 & 6.9 & 4.1 \\
\hline \multicolumn{5}{|l|}{ Thermal Properties } \\
\hline Thermal conductivity (W/m-K) & $\mathrm{k}$ & 0.83 & 16.6 & 2 \\
\hline Coefficient of thermal expansion $\left(10^{-7} / \mathrm{K}\right)$ & $\alpha$ & 99.6 & 48 & 100 \\
\hline \multicolumn{5}{|l|}{ Mechanical Properties } \\
\hline Poisson's ratio & $v$ & 0.24 & 0.23 & 0.4 \\
\hline Fracture toughness $\left(\mathrm{MPa} / \mathrm{m}^{0.5}\right)$ & $\mathrm{K}_{\mathrm{IC}}$ & 0.6 & 2 & 0.51 \\
\hline Young's modulus (GPa) & $\mathrm{E}$ & 70 & 308 & 109 \\
\hline $\begin{array}{l}\text { Thermal Shock Parameter } \\
\text { (for } 25-\mu \mathrm{m} \text { flaw size) }(\mathrm{W} / \mathrm{m}-\mathrm{K})\end{array}$ & $\mathrm{R}_{\mathrm{T}}$ & 61 & 3780 & 63 \\
\hline
\end{tabular}

differences in saturation fluence. Thus, the three different gain media produced different output energies per beamlines and required different numbers of beamlines to meet the overall $3 \mathrm{w}$ output energy requirement of $2.2 \mathrm{MJ}$.

Values of amplifier gain and stored energy were scanned to determine near-minimum values needed to produce $1 \omega$ output fluences. For each value of amplifier gain and stored energy tested, the injected energy from the front end was adjusted until the maximum output energy and extraction efficiency were obtained. Next, with stored energy set at the nearminimum value, the average gain coefficient was adjusted to determine the minimum value consistent with nonlinear phase shift $<2$ radians, for the desired pulse shape. Nonlinear phase shift is limited to avoid beam breakup into small intensity features, which can degrade beam quality and increase damage risk. Using the minimum value of gain coefficient has the advantage of minimizing amplified spontaneous emission loss, which increases nonlinearly with gain coefficient. As gain coefficient was adjusted, the total thickness of glass in the amplifier was also adjusted, to keep overall gain and stored energy constant.

Following the extraction calculations, pumping calculations were performed. First, the average ion doping concentration in the gain medium was scanned until the desired absorption efficiency was obtained (for APG-1), or until the product of the absorption efficiency and the transparency efficiency factor was maximized (for Yb:YAG and for Yb:S-FAP). The transparency efficiency factor, which is important for quasi-three-level lasers, accounts for absorption of the extracting beam by the lower laser level, which increases as doping concentration increases. Using trial values for pump-pulse duration, slab thickness and number of slabs, doping concentrations of individual slabs were adjusted until all slabs had 
approximately the same gain. Diode pump power was adjusted to determine the value required to produce the specified gain. Pump pulse duration was scanned, to determine efficiency vs. diode pump-power curves. If necessary, calculations were repeated, using adjusted slab thicknesses and slab counts, to ensure that thermal stresses in slabs were a small fraction (less than 25\%) of the estimated yield stress.

Calculations were performed for average slab temperatures of $326 \mathrm{~K}$ for Nd-doped APG-1 slabs, at temperatures of $150 \mathrm{~K}, 175 \mathrm{~K}, 200 \mathrm{~K}$, and $232 \mathrm{~K}$ for $\mathrm{Yb}: \mathrm{YAG}$ slabs and at a temperature of $295 \mathrm{~K}$ for $\mathrm{Yb}$ :S-FAP. Values for gain-medium parameters used in our calculations are presented in Table 2. For APG-1, emission cross section and saturation fluence have been adjusted slightly to account for the difference between average slab temperature and room temperature [40,41], at which the spectroscopic properties of APG-1 were determined. Also, for APG-1, saturation fluence was adjusted to compensate for observed discrepancies between Frantz-Nodvik predictions and the results of saturated gain measurements for Nd glasses. It is believed that these discrepancies arise from the effects of inhomogeneous broadening, which occur in the glass matrix. Since no saturated gain measurements at high fluence have been reported for APG-1 glass, we used a correction factor of 0.9 , which is the average value reported in the literature for eight different phosphate laser glasses [42-45]. For Yb:YAG, fits were made to reported temperature-dependent properties, including emission cross section and saturation fluence, radiative lifetime, and thermal conductivity [32,46-48], to allow modeling at arbitrary temperatures between $77 \mathrm{~K}$ and $250 \mathrm{~K}$. Table 2 gives values for $\mathrm{Yb}$ :YAG only at the operating temperature that gave the highest efficiency, which was $200 \mathrm{~K}$.

\section{Results}

Calculated laser efficiency is plotted vs. peak diode power in Fig. 2. Generally, as peak diode power increases, the pump pulselength required to produce the necessary gain and stored energy becomes shorter, which in turn causes decay loss to decrease and efficiency to increase. Since diodes are a major cost factor, the optimum design point for LIFE beamlines is not at the highest efficiency. Rather, it is a point that balances costs for adding diodes against costs for generating more electrical power that is recycled to the laser. Thus, the baseline design chosen for the LIFE laser system, which uses Nd:APG-1 glass, has $\sim 15.6 \%$ overall

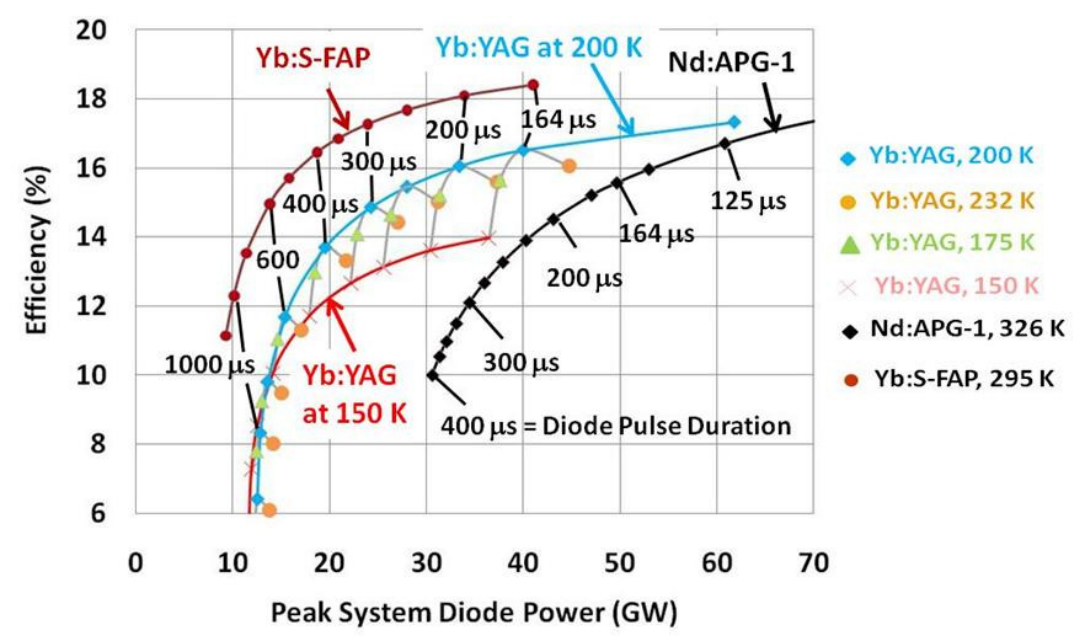

Fig. 2. Efficiency vs. peak system diode power for beamlines using Nd:APG-1, Yb:YAG and Yb:S-FAP laser slabs. Since output fluence and energy per beamline depends upon the gain medium and operating temperature, the number of beamlines was varied to keep the total output pulse energy at $3 \mathrm{w}$ fixed, at $2.2 \mathrm{MJ}$. See text for details. 
Table 3. Beamline Properties

\begin{tabular}{|c|c|c|c|}
\hline Property & APG-1 & Yb:YAG & Yb:S-FAP \\
\hline Average Slab Temperature (K) & 326 & 200 & 295 \\
\hline Peak diode power for $2.2 \mathrm{MJ}$ system (GW) & 50 & 27 & 16 \\
\hline Laser efficiency, including cooling power & 15.6 & 15.5 & 15.7 \\
\hline Laser efficiency, without cooling & 17.9 & 20.8 & 18.56 \\
\hline Cooling Efficiency Factor & 0.87 & 0.74 & 0.85 \\
\hline Number of beamlines used to produce $2.2 \mathrm{MJ}$ & 384 & 390 & 500 \\
\hline $1 \omega$ output energy / beamline $(\mathrm{kJ})$ & 8.1 & 7.9 & 6.3 \\
\hline $3 \omega$ output energy / beamline $(\mathrm{kJ})$ & 5.8 & 5.7 & 4.7 \\
\hline $1 \omega$ output fluence $(\mathrm{J} / \mathrm{cm} 2)$ & 15.0 & 14.6 & 11.5 \\
\hline Number of laser slabs / beamline & 40 & 14 & 40 \\
\hline Slab thickness $(\mathrm{cm})$ & 1.0 & 2.0 & 1.0 \\
\hline Average ion doping concentration $\left(10^{19} / \mathrm{cm}^{3}\right)$ & 7.5 & 3.5 & 0.8 \\
\hline Peak diode irradiance incident on slabs $\left(\mathrm{kW} / \mathrm{cm}^{2}\right)$ & 55 & 30 & 13.4 \\
\hline Diode pulse duration $(\mu \mathrm{s})$ & 164 & 250 & 500 \\
\hline Central pump-diode wavelength (nm) & 877 & 937 & 898 \\
\hline Average gain coefficient $(1 / \mathrm{cm})$ & 0.083 & 0.14 & 0.11 \\
\hline Average thermal power density in laser slabs $(\mathrm{W} / \mathrm{cm} 3)$ & 2.2 & 1.37 & 1.3 \\
\hline Surface-to-center temperature rise in laser slabs $(\mathrm{K})$ & 35 & 4.1 & 8.0 \\
\hline
\end{tabular}

efficiency and uses $\sim 50 \mathrm{GW}$ peak diode power, at a $\sim 164-\mu$ s pulselength. As Fig. 2 shows, higher efficiency is possible but would require the purchase of additional diodes.

The highest predicted efficiencies for $\mathrm{Yb}: \mathrm{YAG}$ are at an average slab operating temperature of $200 \mathrm{~K}$. This optimum temperature occurs as a result of tradeoffs between several temperature-dependent factors. As temperature is increased, extraction efficiency falls due to greater absorption by the thermally-populated lower laser level and due to reduced emission cross section, which causes saturation fluence to be higher. On the other hand, as temperature is increased, ASE losses become smaller, due to the reduced emission cross section, and electrical power consumption by the refrigeration system becomes smaller, due to the increase in coefficient of performance for the refrigeration system. At the LIFE baseline efficiency of $\sim 15.6 \%$,Yb:YAG (at $200 \mathrm{~K}$ ) requires $\sim 45 \%$ fewer diodes than Nd:APG-1. Fewer diodes are required primarily because of the longer radiative lifetime of Yb:YAG (995 $\mu$ s vs. $361 \mu$ s). In comparison, Yb:S-FAP requires $68 \%$ fewer diodes than Nd:APG-1, also at an efficiency of 15.6\%. Yb:S-FAP has longer radiative lifetime than Yb:YAG (1100 $\mu$ s vs. 995 $\mu \mathrm{s})$. Additionally, radiation trapping increases storage lifetime relative to radiative lifetime by about $45 \%$, compared with $\sim 20 \%$ for Yb:YAG. However, the primary reason that Yb:S-FAP requires fewer diodes than $\mathrm{Yb}: \mathrm{YAG}$ is operation at room temperature, which gives a significant efficiency boost due to reduced refrigerator power.

As indicated above, Table 1 gives efficiency breakdowns for Nd:APG-1, Yb:YAG and $\mathrm{Yb}: \mathrm{S}-\mathrm{FAP}$ beamline designs that have approximately the same overall laser electrical-tooptical efficiency of $\sim 15.6 \%$. Absorption efficiencies for Yb:YAG and Yb:S-FAP are lower than for Nd:APG-1, since their ion concentrations were limited to avoid excessive reductions in transparency for the extracting beam. Nd:APG-1 is not significantly affected by transparency, as $\mathrm{Nd}^{3+}$ is a four-level laser. Extraction efficiency for Nd:APG-1 is lower than for $\mathrm{Yb}: \mathrm{YAG}$ and $\mathrm{Yb}: \mathrm{S}-\mathrm{FAP}$ due to the application of the correction factor for saturation fluence to account for inhomogeneous broadening, as explained above. Overall electrical-tooptical conversion efficiency is higher for Yb:YAG than for either Nd:APG-1 or Yb:S-FAP, when the electrical power needed for cooling the laser is not included. The effect on efficiency of including cooling power is substantially greater for Yb:YAG than for Nd:APG-1 and $\mathrm{Yb}: \mathrm{S}-\mathrm{FAP}$, due to operation of $\mathrm{Yb}: \mathrm{YAG}$ at reduced temperature.

Table 3 provides addition information on APG-1, Yb:YAG $(200 \mathrm{~K})$ and Yb:S-FAP beamline designs. The $1 \omega$ output fluence and energy per beamline become progressively 
smaller as one progresses across the table, due to falling saturation fluence and corresponding reduction in the square-pulse-limited output fluence. As output fluence and energy become smaller, a larger number of beamlines are needed to produce the required pulse energy to drive fusion reactions. For example, the laser systems we analyzed, which produce $2.2 \mathrm{MJ}$ at $3 \omega$ and which use slabs of Nd:APG-1, Yb:YAG and Yb:S-FAP, require 384, 390 and 500 beamlines, respectively. It should be noted that the numbers of beamlines needed could have been changed by adjusting the aperture size.

\section{Summary}

We have performed a side-by-side comparison of the efficiency of Nd:APG-1, Yb:YAG and $\mathrm{Yb}: \mathrm{S}$-FAP beamlines that have been designed for use in laser inertial fusion energy (LIFE) power plants. Our modeling accounts for 18 different efficiency factors, each representing an energy-transfer step or an energy loss mechanism. When multiplied together, these efficiency factors give the overall electrical-to-optical conversion efficiency of the laser. Two loss factors, one of which represents losses due to spontaneous decay while the other of represents losses due to amplified spontaneous emission, depend on the gain medium chosen as well as on the duration of the pump pulse. These losses can be reduced by using a higher diode peak power to pump the laser slabs for a shorter period of time, while still attaining the necessary population inversion. Provided sufficient numbers of diodes are used, beamlines using Nd:APG-1, Yb:YAG or Yb:S-FAP can achieve an electrical-to-optical conversion efficiency of at least $12-16 \%$, well within the desirable range for the LIFE power-plant application. Due largely to differences in storage lifetime, the three different gain media require different numbers of diodes. For example, to achieve an overall electrical-to-optical conversion efficiency of about $15.6 \%$, laser systems using gain media of Nd:APG-1, Yb:YAG and Yb:SFAP require relative peak diode powers of $1,0.55$ and 0.32 , respectively. Additionally, due to differences in saturation fluence, the three different gain media operate at different $1 \mathrm{w}$ fluences and require different numbers of beamlines.

\section{Acknowledgments}

This work was performed under the auspices of the U.S. Department of Energy by Lawrence Livermore National Laboratory under Contract DE-AC52-07NA27344. 


\title{
Thermal birefringence and depolarization compensation in glass-based high-average-power laser systems
}

\author{
Amber L. Bullington*, Steven B. Sutton, Andy J. Bayramian, John A. Caird, Robert J. Deri, Al C. \\ Erlandson, Mark A. Henesian \\ Lawrence Livermore National Laboratory, 7000 East Avenue, Livermore, CA, USA 94550
}

\begin{abstract}
Thermally induced birefringence can degrade the beam quality in high-average-power laser systems with doped-glass substrates. In this work, we compare glass-laser slab amplifiers at either Brewster's angle or normal incidence and discuss trade-offs between both designs. Numerical simulations show the impact of thermally induced depolarization in both amplifier systems. A non-uniform temperature profile and the resultant mechanical stress leads to depolarization that worsens as the beam propagates through the slab-amplifier chain. Reflective losses for depolarized light at Brewster's angle cannot be compensated and degrade beam quality. This motivates the selection of normally incident slab amplifiers, which facilitates birefringence compensation.

Tolerances for birefringence compensation of two matched normal-incidence glass-slab amplifiers balanced by a quartz rotator are also investigated. Imbalances in thermal load, relative amplifier position and beam magnification between amplifiers show the highest depolarization sensitivity and establish limits for manufacturing tolerances and amplifier design.
\end{abstract}

Keywords: thermal birefringence, depolarization, high-average-power lasers

\section{INTRODUCTION}

Thermally induced stress birefringence can present challenges for high-average-power lasers, including those with glass as the host material. Non-uniform heating of a glass slab creates thermal gradients at the pump-beam edges resulting in stress birefringence that depolarizes the beam. Excessive birefringence can have negative effects on beam quality, especially when polarized output is required.

Laser amplifiers with glass slabs positioned at either Brewster's angle or normal incidence are both susceptible to thermal birefringence. P-polarized reflectivity is zero at Brewster's angle, providing the advantage of not requiring antireflective (AR) coatings that may damage under high-power operation. However, any light depolarized in a Brewster's angle slab amplifier experiences a high reflective loss. In this work, we investigate thermally induced depolarization in Brewster's angle slabs to determine beam quality degradation and compare the results to slabs at normal incidence. The results motivate the use of AR-coated normal incidence slabs with birefringence compensation.

A balanced-amplifier compensation scheme is also presented. Two amplifiers, equally pumped with the same number of slabs, are birefringence-compensated via a polarization rotator. Imbalances in the amplifiers giving the highest residual depolarization are discussed. These potential asymmetries determine the tightest tolerances for amplifier design.

*bullington1@1lnl.gov; phone 925-423-7589; fax 925-423-6195

High Power Lasers for Fusion Research, edited by Abdul A. S. Awwal, A. Mike Dunne, Hiroshi Azechi, Brian E. Kruschwitz, Proc. of SPIE Vol. 7916 79160V · @ 2011 SPIE · CCC code: 0277-786X/11/\$18 - doi: 10.1117/12.876531 


\section{ANALYZING THERMAL BIREFRINGENCE}

Thermal birefringence is modeled using a multi-step method that links data from finite-element analysis to a ray-tracing program for analyzing beam depolarization. Finite-element modeling of the temperature profile and stress are performed for a given heat deposition profile. These results serve as inputs to a ray-tracing program that calculates the Jones matrices for determining the beam depolarization as a function of position for a single slab. An additional script multiplies these Jones matrices to determine the beam depolarization in a multi-slab amplifier. A flowchart of the analysis is given in Figure 1. The finite-element thermal and stress analyses as well as the depolarization ray-trace model are based on validated codes that have been tested and previously published in the literature [1]. Multiplying Jones matrices for multiple-slab amplifiers is performed with a MATLAB script.

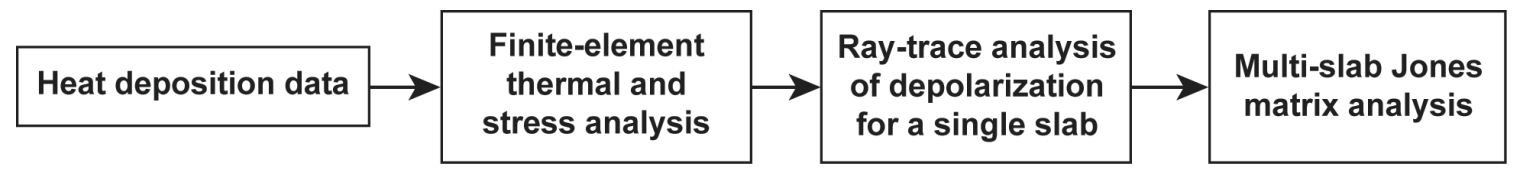

Figure 1. A flowchart summarizes the numerical analysis of the thermal birefringence.

The heat deposition is given by an analytical super-Gaussian pump profile of order $n$ and half-width $w$ that can be written as

$$
S G(x, y)=\exp \left[-\left(x^{n}+y^{n}\right) / w^{n}\right] .
$$

The depolarization ray-trace analysis treats each finite element composing the slab as a linear retarder. At each element, rays are traced through the thickness of the slab to determine the Jones matrix for each spatial coordinate. The Jones matrix for given $x$ and $y$ coordinates on a single slab can be written as

$$
R(\psi, \delta)=\left[\begin{array}{cc}
\cos [\delta(x, y) / 2] & 0 \\
0 & \cos [\delta(x, y) / 2]
\end{array}\right]+i\left[\begin{array}{lr}
-\cos [2 \psi(x, y)] \sin [\delta(x, y) / 2] & -\sin [2 \psi(x, y)] \sin [\delta(x, y) / 2] \\
-\sin [2 \psi(x, y)] \sin [\delta(x, y) / 2] & \cos [2 \psi(x, y)] \sin [\delta(x, y) / 2]
\end{array}\right]
$$

where

$$
\delta(x, y)=\frac{2 \pi l}{\lambda}\left(n_{x^{\prime}}-n_{y^{\prime}}\right)
$$

is the retardation and $\psi(x, y)$ is the rotation angle of the retarder axis [2-4]. $l$ is the distance traversed through the medium and $n_{x}$, and $n_{y}$, are the refractive indices along the axes in the coordinate frame of the slab element being ray-traced. The depolarization for a multi-slab amplifier is calculated from the product of the Jones matrices comprising the individual slabs. For an amplifier with $n$ identical slabs, it can be shown that the amplifier's Jones matrix is equal to

$$
R(\psi, \delta)^{n}=\left[\begin{array}{cc}
\cos [n \delta(x, y) / 2] & 0 \\
0 & \cos [n \delta(x, y) / 2]
\end{array}\right]+i\left[\begin{array}{cc}
-\cos [2 \psi(x, y)] \sin [n \delta(x, y) / 2] & -\sin [2 \psi(x, y)] \sin [n \delta(x, y) / 2] \\
-\sin [2 \psi(x, y)] \sin [n \delta(x, y) / 2] & \cos [2 \psi(x, y)] \sin [n \delta(x, y) / 2]
\end{array}\right],
$$

where the retardation is multiplied by the number of slabs, $n$. The depolarization for a multi-slab amplifier is given by $[2,3]$

$$
\text { Depol }=\sin [2 \psi(x, y)]^{2} \sin [n \delta(x, y) / 2]^{2} .
$$

For analyses in this paper, the incident light is assumed to be p-polarized. The depolarization is given by the power converted to s-polarization. When circularly polarized light is propagated through an amplifier system, the output field is multiplied by an analytical quarter-wave plate to convert to linear polarization. 


\section{AMPLIFIER WITH BREWSTER'S ANGLE SLABS}

Amplifiers constructed with Brewster's angle slabs have the advantage of not requiring AR coatings. While p-polarized light experiences no reflective loss at Brewster's angle, s-polarized light has a $16 \%$ reflective loss at each slab interface. Any depolarized light sees a power loss from multiple reflective surfaces. The loss for a 44-slab amplifier is analyzed in this section.

\subsection{Analysis with a theoretical super-Gaussian pump profile}

Figure 2a shows the temperature distribution at the slab-center for a super-Gaussian pump beam of order $n=20$ and half-width $w=20 \mathrm{~cm}$ incident on a $42 \mathrm{~cm}$ by $42 \mathrm{~cm}$ glass slab. The slab thickness is $1 \mathrm{~cm}$. The pump beam is normally incident on the Brewster's angle slab. The front and back faces of the slab are cooled with flowing gas. Since the slabs are oriented at Brewster's angle, the transmitted beam is rectangular with dimensions $22 \mathrm{~cm}$ by $40 \mathrm{~cm}$. The $40 \mathrm{~cm}$ by 40 $\mathrm{cm}$ inner window defines the extent of the ray-trace for depolarization analysis. Any roll-off of the transmitted beam is assumed to occur in the $2-\mathrm{cm}$ border region of the slab. The temperature gradients from pump-beam roll-off lead to shear stress extending into the slab in Figure $2 \mathrm{~b}$. The maximum heat deposition is $1.66 \mathrm{~W} / \mathrm{cm}^{3}$.

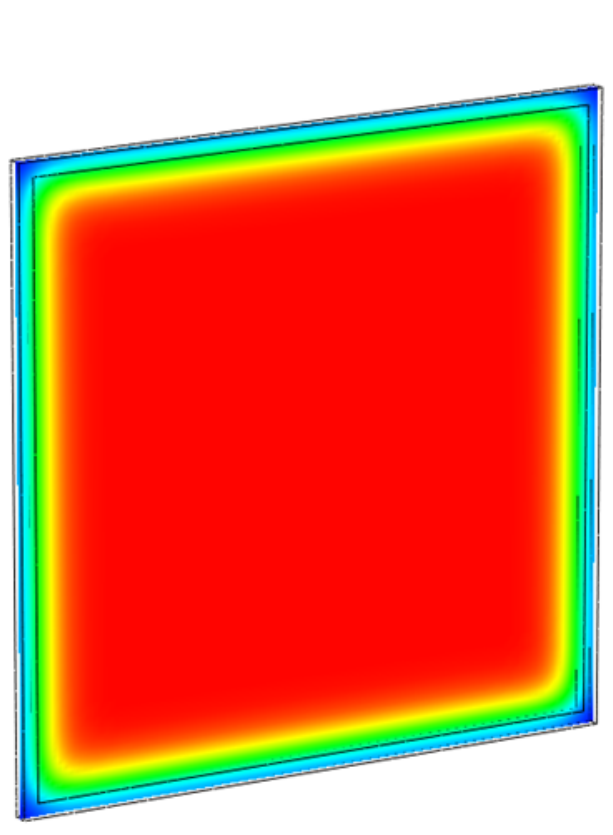

a

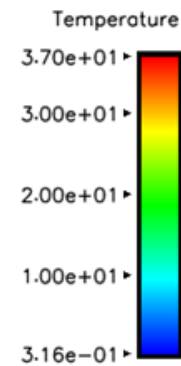

Figure 2. Plot (a) shows the temperature profile in degrees Celcius at the center of the slab with heat deposition data determined by a super-Gaussian pump profile. Plot (b) shows the resultant shear stress at slab-center in $\mathrm{N} / \mathrm{cm}^{2}$. The slab dimensions are $42 \mathrm{~cm}$ by $42 \mathrm{~cm}$. The inner window is $40 \mathrm{~cm}$ by $40 \mathrm{~cm}$ and defines the extent of the beam for ray-trace analysis.

The depolarization for a single Brewster's angle slab is shown in Figure 3 with a maximum depolarization of 54\%. The $\mathrm{x}$-dimension is shortened because of the Brewster's angle orientation of the slab. 

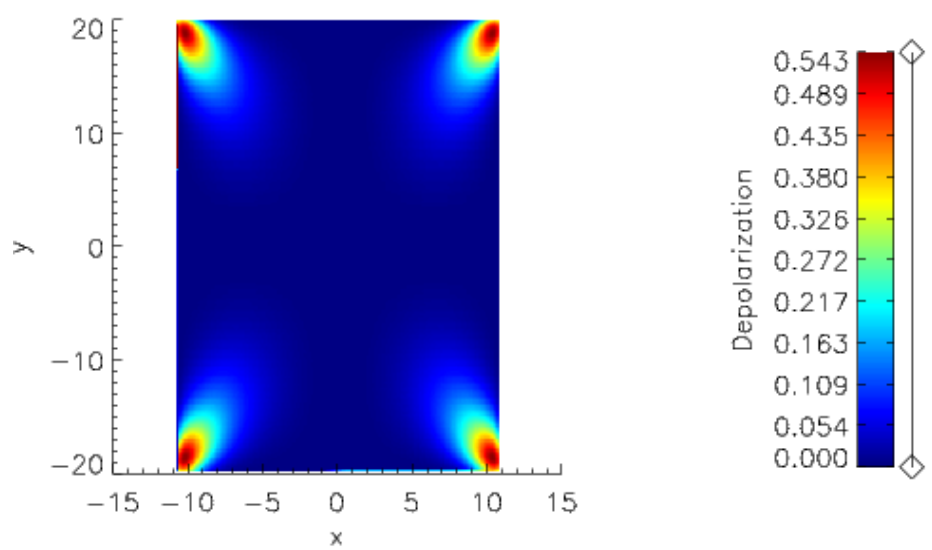

Figure 3. A map of depolarization is shown given the temperature and stress from Figure 2. The map in the $\mathrm{x}$ dimension is reduced due to the Brewster's angle orientation of the slab.

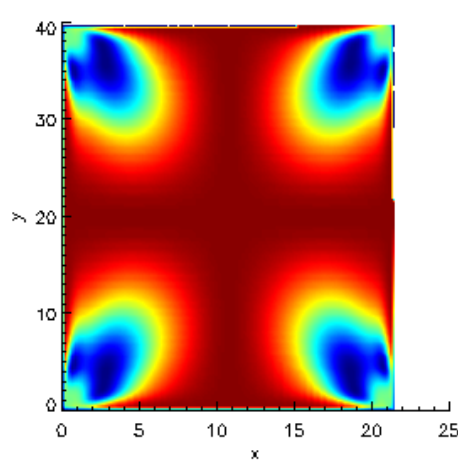

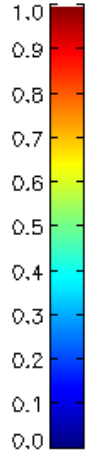

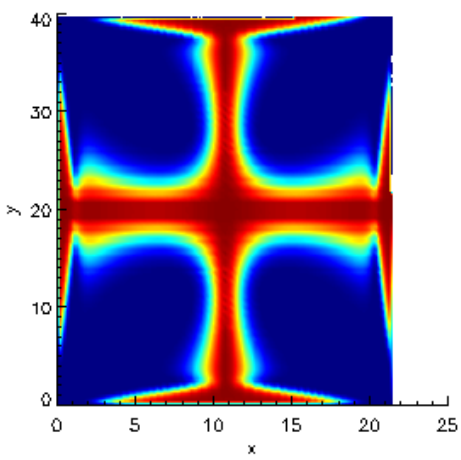

$\mathrm{b}$

Figure 4. Image (a) shows the transmitted p-polarized light after traversing four Brewster's angle slabs. Image (b) shows the transmitted beam after double-passing a 44-slab amplifier ( 88 passes through Brewster's angle slabs). Both images use temperature and stress data from Figure 2 and include the $16 \%$ per interface reflective loss seen by the depolarized light. Gain is not included.

Figure 4 shows the p-polarized transmitted beam after traversing (a) four slabs and (b) a double-pass of an amplifier with 44 slabs. Gain is not included in the calculation. Figure $4 \mathrm{~b}$ demonstrates that traversing multiple slabs compounds the depolarization loss. By double-passing a Brewster-slab amplifier that includes traversing 44 individual slabs twice and encountering 176 reflective interfaces, the depolarized light at the slab corners is lost. This creates a transmitted beam with an iron-cross shape instead of the desired flat-beam profile across the slab aperture. The fraction of power remaining in p- and s-polarization for single- and double-passing the amplifier is given in Table 1 . Only $0.3 \%$ of the spolarized light and $26 \%$ of the p-polarized light remains after a double pass. Lost power as a function of the number of slabs traversed during a double pass is shown in Figure 5. For a double-passing a 44-slab amplifier, the lost power reaches $75 \%$. Transmitted-beam degradation must be overcome for Brewster's angle slabs to be a viable option for highaverage-power laser systems.

Table 1. The fraction of average power in each polarization is given after a single- and double-pass of a 44-slab amplifier. $16 \%$ reflective loss for s-polarization at each interface is included.

\begin{tabular}{|c|c|c|}
\hline & Single Pass & Double Pass \\
\hline P-polarized light & 0.3285 & 0.2600 \\
\hline S-polarized light & 0.0080 & 0.0030 \\
\hline
\end{tabular}




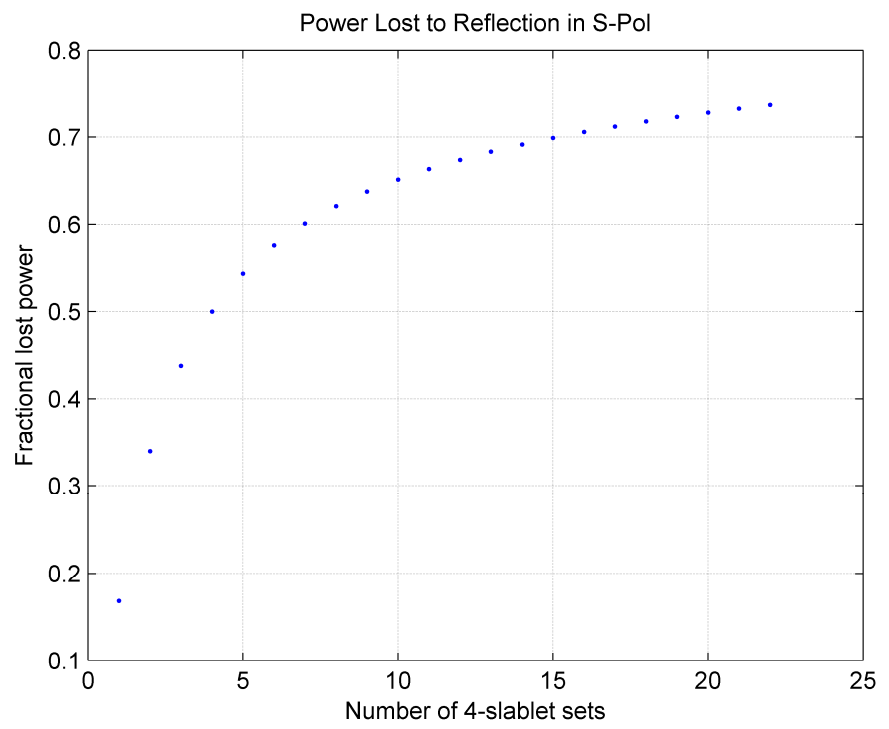

Figure 5. The fraction of power lost from reflected s-polarized light is shown as a function of the number of slabs traversed for a double-pass of the amplifier.

\subsection{Varying the super-Gaussian pump profile}

A uniform pump profile with a sharp roll-off at the edges may minimize depolarization from thermal birefringence. Super-Gaussian pump profiles of varying half-widths, $w$, and order, $n$, are tested with Brewster's angle slabs. The average depolarization for a $42 \mathrm{~cm}$ by $42 \mathrm{~cm}$ slab is shown in Table 2. Overfilling the pump beam on the slab reduces the magnitude of the temperature gradients from pump roll-off, thereby reducing the depolarization. Increasing the order of the super-Gaussian from $n=20$ to $n=30$ increases the rate of pump roll-off. However, Table 2 shows that overfilling the pump beam on the slab is more beneficial than increasing the pump roll-off.

Table 2. Individual slab depolarization is given for various super-Gaussian pump profiles on a $42 \mathrm{~cm} \mathrm{x} 42 \mathrm{~cm}$ slab.

\begin{tabular}{|c|c|c|}
\hline Super-Gaussian & $\begin{array}{c}\text { Single-slab average } \\
\text { depolarization }\end{array}$ & Pump-beam overfill loss \\
\hline$w=19 \mathrm{~cm}, \mathrm{n}=20$ & 0.1567 & $0.2 \%$ \\
\hline$w=19 \mathrm{~cm}, \mathrm{n}=30$ & 0.1517 & $0.01 \%$ \\
\hline$w=20 \mathrm{~cm}, \mathrm{n}=20$ & 0.0797 & $2.3 \%$ \\
\hline$w=21 \mathrm{~cm}, \mathrm{n}=20$ & 0.0244 & $7.4 \%$ \\
\hline$w=22 \mathrm{~cm}, \mathrm{n}=20$ & 0.0053 & $14 \%$ \\
\hline
\end{tabular}

From Table 2, a super-Gaussian half-width of $22 \mathrm{~cm}$ gives an average depolarization of $0.5 \%$ for a single Brewster's angle slab. However, the pump power lost from overfilling the super-Gaussian profile on the slab is $14 \%$. The depolarized light lost to reflection when double-passing 44 slabs is $55 \%$ when $w=22 \mathrm{~cm}$ compared to $75 \%$ for $w=20$ $\mathrm{cm}$ observed in Figure 5.

Reducing depolarization in a Brewster-slab amplifier involves sacrificing pump efficiency by overfilling the slab with pump light while still suffering high losses from reflected depolarized light. Losing pump efficiency to reduce depolarization does not provide sufficient benefit to improving performance of a Brewster's angle slab amplifier. As a result, normally incident slab amplifiers are considered over Brewster's angle slabs. 


\section{AMPLIFIER WITH NORMAL-INCIDENCE SLABS}

Figure 6 shows the depolarization pattern, excluding reflective loss, for a normally incident slab pumped by a superGaussian beam of order 20 with a half-width of $11.5 \mathrm{~cm}$. The incident beam is assumed to have the same dimensions as the pump beam. The slab dimensions are $26 \mathrm{~cm}$ by $26 \mathrm{~cm}$ with a heat load of $2.27 \mathrm{~W} / \mathrm{cm}^{3}$. The slab thickness is $1 \mathrm{~cm}$ and the slab faces are gas-cooled. Figure 7 shows the maximum p-polarized transmission after a single pass through a 22 -slab amplifier and includes $0.1 \%$ AR-coating reflective loss at each interface. The periodic variation seen in Figure 7 that resembles an isogyre pattern stems from the phase wrapping that occurs once the retardation (Eqn. 3 ) reaches $2 \pi$. Note that both $\mathrm{p}$ - and s-polarizations at normal incidence experience equal reflective loss as opposed to the Brewster's angle case. Thus, any depolarized light is still present with equal power compared to the power of the desired polarization in the system. Clearly, depolarization is a problem for normal-incidence slabs if polarized output is required. However, equal reflective loss for s- and p-polarized light allows for birefringence compensation.
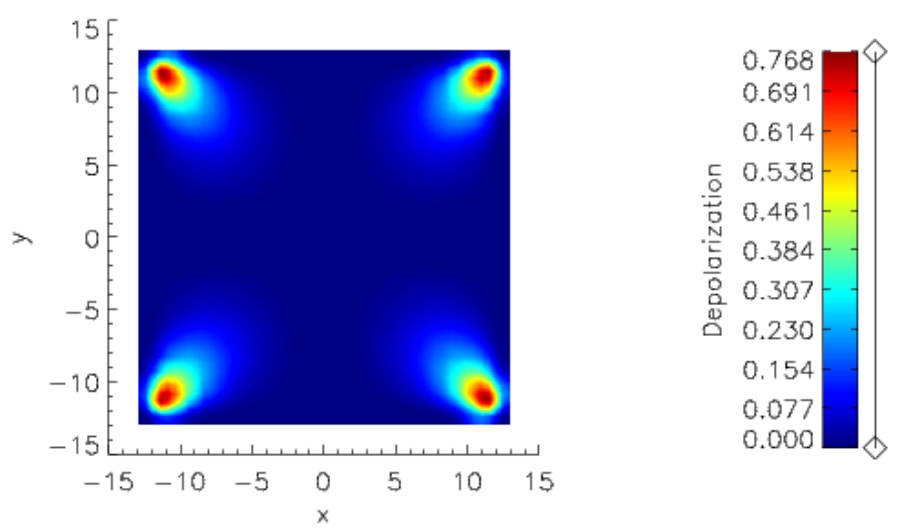

Figure 6. The depolarization for a normally incident slab is shown given a heat load of $2.27 \mathrm{~W} / \mathrm{cm}^{3}$.
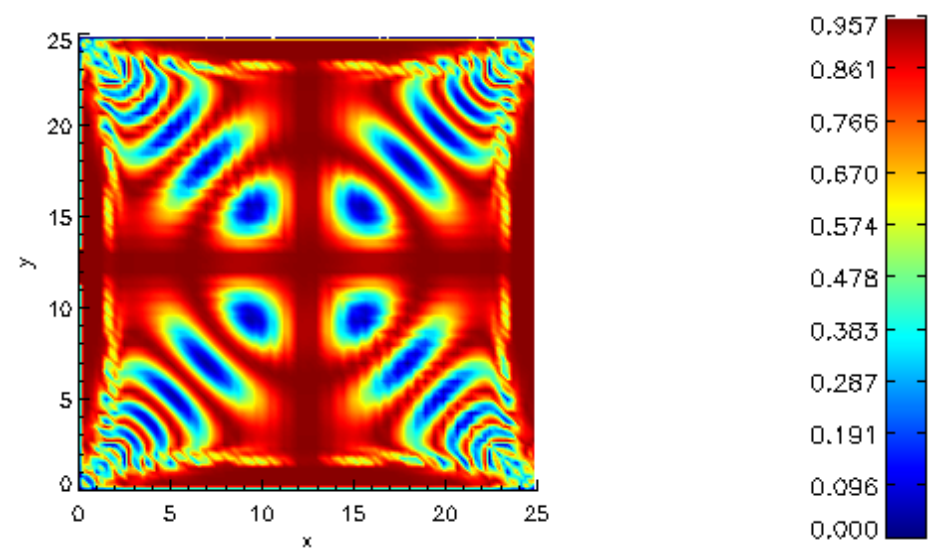

Figure 7. Maximum p-polarized transmission is shown after a single-pass of a 22-slab amplifier. An AR-coating loss of $0.1 \%$ is included at each interface and affects both p- and s-polarizations equally. 


\section{BIREFRINGENCE COMPENSATION}

One method for compensating thermal birefringence involves placing a polarization rotator between two identical amplifiers [5,6]. Figure 8 shows a schematic of a balanced compensation scheme. A quartz rotator rotates the polarization by 90 degrees. This allows any depolarized light to experience an equal and opposite phase retardation in the second amplifier, thereby eliminating depolarization. The incident light is linearly polarized, while the light traversing the amplifiers is converted to circular polarization via a quarter-wave plate to reduce nonlinear focusing related to B-integral [7,8]. Depolarization is analyzed by converting output circular polarization to linear polarization with a Jones matrix for a quarter-wave plate and noting the residual depolarized light. The mirror in Figure 8 may be replaced with a quarter-wave plate for calculating single-pass depolarization to convert the circularly polarized light back to linear polarization.

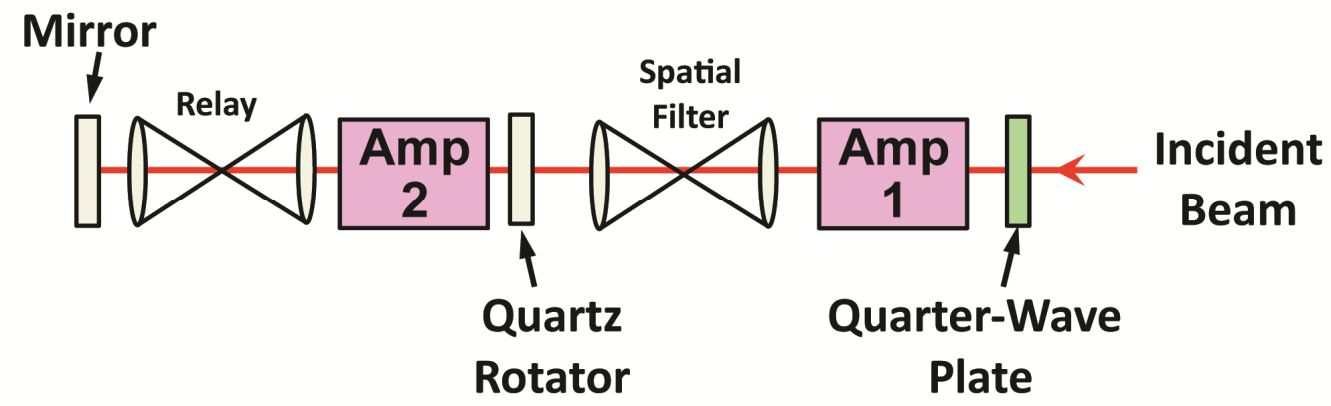

Figure 8. A birefringence compensation scheme using two balanced amplifiers (Amp) and a quartz rotator is shown with relay imaging optics and a spatial filter. A mirror is used to double-pass the amplifier, but may be exchanged for a quarter-wave plate for single-pass depolarization analysis. The incident light is linearly polarized but is converted to circular polarization with a quarter-wave plate.

Imbalances between amplifiers and residual depolarization are addressed in this section. Differences in amplifiers 1 and 2 that have the tightest tolerances are addressed, including a heat imbalance between amplifiers, one amplifier offset in position with respect to the other, and a beam magnification error in one amplifier. Thermal loading in the quartz rotator is also discussed.

\subsection{Birefringence compensation with increased heat load in one amplifier}

Table 3 lists the percentage of depolarized light remaining after compensation when amplifier 2 has a higher heat load than amplifier 1. Double-passing the system with only $2 \%$ higher heat in one amplifier leads to $5.4 \%$ residual depolarization. To keep any residual depolarization below a nominal value of $2 \%$, the heat load in each amplifier must agree to within $1 \%$.

Table 3. Depolarization after birefringence compensation with amplifier 2 running hotter than amplifier 1.

\begin{tabular}{|c|c|c|}
\hline $\begin{array}{c}\text { Heat Increase, } \\
\text { Amplifier 2 }\end{array}$ & $\begin{array}{c}\text { Single-Pass } \\
\text { Depolarization }\end{array}$ & $\begin{array}{c}\text { Double-Pass } \\
\text { Depolarization }\end{array}$ \\
\hline $1 \%$ Hotter & $0.56 \%$ & $1.56 \%$ \\
\hline $2 \%$ Hotter & $2.1 \%$ & $5.4 \%$ \\
\hline
\end{tabular}

\subsection{Birefringence compensation with a position offset for one amplifier}

If one amplifier is offset in position with respect to the other, significant depolarization can remain after compensation. Amplifier 2 is offset laterally with respect to amplifier 1 by varying amounts as listed in Table 4 . An offset of only $1 \mathrm{~mm}$ gives a single-pass residual depolarization of $4 \%$. 
Table 4. Depolarization with one amplifier offset laterally in position with respect to the other.

\begin{tabular}{|c|c|}
\hline Position Offset & Single-Pass Depolarization \\
\hline $200 \mu \mathrm{m}$ & $0.71 \%$ \\
\hline $400 \mu \mathrm{m}$ & $2.3 \%$ \\
\hline $600 \mu \mathrm{m}$ & $3.5 \%$ \\
\hline $800 \mu \mathrm{m}$ & $3.9 \%$ \\
\hline $1 \mathrm{~mm}$ & $4.0 \%$ \\
\hline
\end{tabular}

Figure 9 shows the transmitted beam images for amplifier offsets of $200 \mu \mathrm{m}$ and $1 \mathrm{~mm}$. The beam distorts around the edges with increasing offset. AR-coating loss of $0.1 \%$ is included in the plots. Each amplifier must be positioned to less than $1 \mathrm{~mm}$ accuracy to keep lateral-offset depolarization to a minimum.

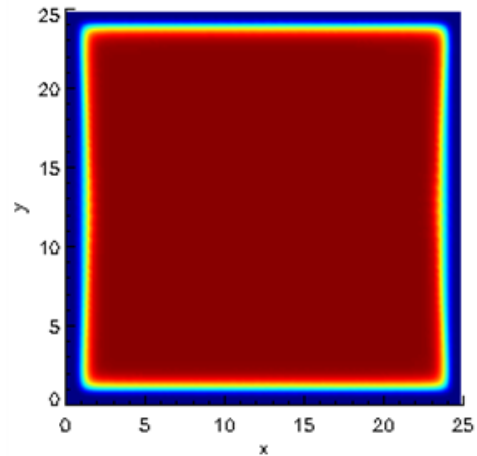

(a) $200 \mu \mathrm{m}$ offset
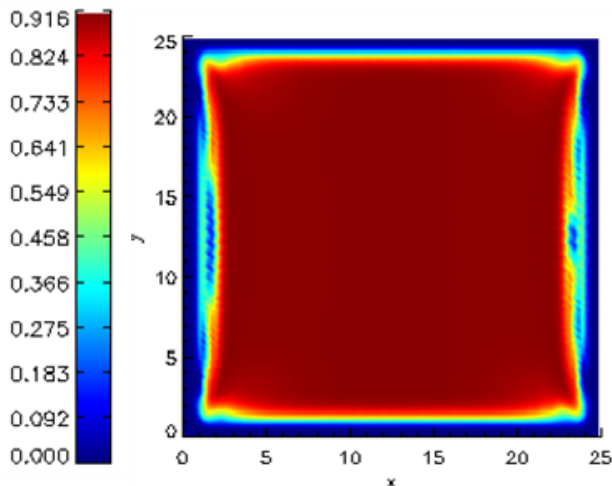

(b) $1 \mathrm{~mm}$ offset

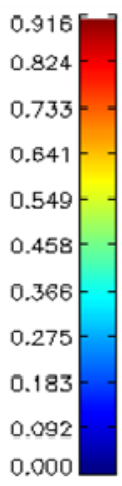

Figure 9. Transmitted beam images from a single pass of two compensated amplifiers with one amplifier offset to the right by (a) $200 \mu \mathrm{m}$ and (b) $1 \mathrm{~mm}$. An AR-coating loss of $0.1 \%$ is included.

\subsection{Birefringence compensation with a magnification error}

The beam must be reimaged from one amplifier into the other via a relay telescope. If the telescope does not relay the beam with perfect one-to-one imaging, the beam will sample a larger area of one amplifier compared to the other amplifier. Table 5 gives the residual depolarization as a function of increase in beam diameter. The depolarization increases rapidly with magnification error. This offers a guideline for determining manufacturing tolerances on the relay telescope elements.

Table 4. Depolarization from a relay telescope magnification error. The second amplifier experiences the given increase in beam diameter.

\begin{tabular}{|c|c|}
\hline Beam Diameter Increase & Single-Pass Depolarization \\
\hline $200 \mu \mathrm{m}$ & $0.43 \%$ \\
\hline $400 \mu \mathrm{m}$ & $1.7 \%$ \\
\hline $600 \mu \mathrm{m}$ & $3.5 \%$ \\
\hline $1 \mathrm{~mm}$ & $7.5 \%$ \\
\hline
\end{tabular}




\subsection{Thermal loading of the quartz rotator}

Thermal variation of rotation in quartz has been investigated by Chandrasekhar $[9,10]$. At the operating wavelength of $1053 \mathrm{~nm}$, the rotation per $\mathrm{mm}, \rho$, has a temperature dependence, $d \rho / d T$, of $7.643 \times 10^{-4} \mathrm{deg} / \mathrm{mm} / \mathrm{K}$. This equates to a variation in $\rho$ of $0.11 \%$ for a 10 degree increase in temperature. Thus, temperature variation of polarization rotation in quartz at $1053 \mathrm{~nm}$ is quite small and should not pose a problem for birefringence compensation.

\section{CONCLUSION}

Depolarization losses that are overcome at the expense of efficiency make Brewster's angle slab amplifiers a less attractive option than normal-incidence slab amplifiers. Normally incident slab amplifiers allow for depolarization losses to be compensated with balanced amplifiers and a quartz rotator. By meeting the system design tolerances determined by residual depolarization discussed in section 5, the normally incident slab amplifier is a promising option for a highaverage-power laser system.

This work was performed under the auspices of the U.S. Department of Energy by Lawrence Livermore National Laboratory under Contract DE-AC52-07NA27344.

\section{REFERENCES}

[1] Rotter, M., Jancaitis, K., Marshall, C., Zapata, L., Erlandson, A., LeTouze, G., and Seznec, S., "Pump-induced wavefront distortion in prototypical NIF/LMJ amplifiers - modeling and comparison with experiments," Proc. SPIE 3492, 638-659 (1999).

[2] Rotter, M., "An algorithm for depolarization," LLNL internal memo, (1998).

[3] Chen, Y., Chen, B., Patel, M. K. R., and Bass, M., "Calculation of thermal-gradient-induced stress birefringence in slab lasers - I," IEEE J. Quant. Elec. 40(7), 909-916 (2004).

[4] Giuliani, G. and Ristori, P., "Polarization flip cavities: a new approach to laser resonators," Opt. Comm. 35(1) 109$112(1980)$.

[5] Frede, M. Wilhelm, R., Brendel, M., Fallnich, C., Seifert, F., Willke, B., and Danzmann, K., "High power fundamental Mode Nd:YAG laser with efficient birefringence compensation," Opt. Express, 12(15) 3581-3589 (2004).

[6] Frede, M. Wilhelm, R., Gau, R., Brendel, M., Zawischa, I., Fallnich, C., Seifert, F., and Willke, B., "High-power single-frequency Nd:YAG laser for gravitational wave detection," Classical and Quantum Gravity, 21, 895-901 (2004).

[7] Feldman, A., Horowitz, D., and Waxler, R., "Mechanisms for self-focusing in optical glasses," IEEE J. Quantum Electron., QE-9, 1054-1061 (1973).

[8] Auric, D., and Labadens, A. "On the use of a circularly polarized beam to reduce the self-focusing effect in a glass rod amplifier," Opt. Comm., 21, 241-242 (1977).

[9] Chandrasekhar, S. "The Optical Rotary Power of Quartz and its Variation with Temperature," Proceedings of the Indian Academy of Sciences, 35, 103-113 (1951).

[10] Chandrasekhar, S. "The Temperature Variation of the Rotary Power of Quartz from 30 deg to 410 deg C," Proceedings of the Indian Academy of Sciences, 39, 290-295 (1954). 


\title{
Improved Characterization of Transmitted Wavefront Error on CADB ${ }^{\circ}$ Epoxy-Free Bonded Solid State Laser Materials
}

\author{
Nick Traggis $^{{ }^{\mathrm{a}} \mathrm{a}}$, Neil Claussen ${ }^{\mathrm{a}}$, Andy Bayramian ${ }^{\mathrm{b}}$, Kathleen Schaffers ${ }^{\mathrm{b}}$, Bob Deri ${ }^{\mathrm{b}}$ \\ ${ }^{a}$ Precision Photonics Corporation, 3180 Sterling Circle, Boulder, CO 80301 \\ ${ }^{b}$ Lawrence Livermore National Laboratory, 7000 East Ave., L-470, Livermore, CA 94551
}

\begin{abstract}
Current state-of-the-art and next generation laser systems - such as those used in the NIF and LIFE experiments at LLNL - depend on ever larger optical elements. The need for wide aperture optics that are tolerant of high power has placed many demands on material growers for such diverse materials as crystalline sapphire, quartz, and laser host materials. For such materials, it is either prohibitively expensive or even physically impossible to fabricate monolithic pieces with the required size. In these cases, it is preferable to optically bond two or more elements together with a technique such as Chemically Activated Direct Bonding $\left(\mathrm{CADB}^{\circ}\right)$. CADB is an epoxy-free bonding method that produces bulk-strength bonded samples with negligible optical loss and excellent environmental robustness. The authors have demonstrated CADB for a variety of different laser glasses and crystals. For this project, we will bond quartz samples together to determine the suitability of the resulting assemblies for large aperture high power laser optics. The assemblies will be evaluated in terms of their transmitted wavefront error, and other optical properties.
\end{abstract}

Keywords: Optical bonding, quartz rotators, CADB, LIFE

\section{INTRODUCTION}

Several projects are underway worldwide which promise to demonstrate fusion ignition and gain. The first of these to be completed is the National Ignition Facility (NIF) at Lawrence Livermore National Laboratory[1]. ${ }^{1,2}$ In 2010, the team of scientists and engineers operating the NIF will start a campaign to demonstrate fusion ignition, which could serve as the basis for a national program to develop Laser Inertial Fusion Energy (LIFE). A LIFE power generation system will have to meet all of the basic requirements of the NIF including laser requirements, target geometry, target illumination, radiation/hazardous materials handling and safety. In addition, the power generation system will have to address high average power operation of the laser system, average power effects on the target chamber, target injection and tracking, target mass production, blanket and tritium production, and the balance of plant to produce electricity.

A laser driver meeting these basic requirements was formulated based on a self consistent conceptual design for a Laser Inertial Fusion Energy (LIFE) power plant. ${ }^{3-6}$ Although we have used only modest extensions of existing laser technology to ensure near-term feasibility, predicted performance meets or exceeds plant requirements: $2.4 \mathrm{MJ}$ pulse energy produced by 576 beamlines at $15 \mathrm{~Hz}$, with $12 \%$ wall-plug efficiency. High reliability and maintainability are achieved by mounting components in compact line-replaceable units that can be removed and replaced rapidly while other beamlines continue to operate, at up to $\sim 8 \%$ above normal energy, to compensate for neighboring beamlines that have failed. Statistical modeling predicts that laser-system availability can be greater than $99 \%$ provided that components meet reasonable mean-time-between-failure specifications. In our proposed development plan, a prototype beamline is built and tested in $\sim 4$ years and a 288 -beam laser system for the first LIFE demonstration power plant is built and activated in $\sim 13$ years. Timely demonstration of fusion energy is required to meet an expected growth in power plant production and displace carbon based power sources slated for this need.

One of the many optics required for this laser architecture is a large aperture $\left(27 \times 27 \mathrm{~cm}^{2}\right) 90$ degree quartz rotator for the Nd:glass laser beamline. Such an optic is larger than any current quartz crystal, and many years of growth scaling will be required to eventually scale the optic up to full aperture. A potential alternative to growing very large crystals is to optically bond smaller apertures together.

The Chemically Activated Direct Bonding process $(\mathrm{CADB} \AA)$ has been developed by Precision Photonics Corp and results in epoxy-free optical paths that are $100 \%$ optically transparent with negligible scattering and absorptive losses at the interfaces. For YAG and similar materials, the process has been proven to offer bond strength performance

Solid State Lasers XX: Technology and Devices, edited by W. Andrew Clarkson, Norman Hodgson, Ramesh Shori, Proc. of SPIE Vol. 7912, 79121K · C 2011 SPIE · CCC code: 0277-786X/11/\$18 · doi: 10.1117/12.877184 
equivalent to that of bulk. It is thus exceptionally durable, reliable and resistant to changes in laser fluence, temperature, and humidity. ${ }^{7}$ Due to the zero bond line thickness, complete conformance is ensured between the two bonded surfaces, making it ideal for this application. Using the CADB process, a small aperture quartz rotator samples were bonded together to test this alternative.

\section{PREPARATION OF QUARTZ ROTATOR ASSEMBLIES}

To fabricate the bonded assembly, we procured a set of 4 commercial, off-the-shelf small aperture quartz rotators from a separate vendor. As shown in Fig. 1(a), the substrates were $20 \mathrm{~mm} \times 20 \mathrm{~mm} \times 14 \mathrm{~mm}$, with the quartz extraordinary axis oriented normal to each square polished face. We decided to construct one rotator assembly from each pair of small aperture quartz pieces. To allow for a robust evaluation of the bonded interface in the final assembly, we polished the $14 \mathrm{~mm} \times 20 \mathrm{~mm}$ face of each quartz slab at an angle of $15^{\circ}$. The alignment error on this angle as well as the adjacent face $90^{\circ}$ angle were maintained at $<1$ arc minute, which ensured that the quartz e-axes would match up well at the bond line. Axis matching is essential in quartz because of the large optical and thermo-mechanical asymmetries in the material.

We used a special process to polish the quartz bonding surfaces. Our goal was a set of surfaces with low rms roughness, good surface quality, and minimal flatness error at the outer edges (edge roll-off). By reducing edge roll-off, we could increase the usable aperture of the optic and minimize the extent of the delaminated seams that form along the perimeter of the bonded interface. After we finished polishing, we proceeded to bond the individual quartz pieces. We used the CADB process to join the 2 pairs of slabs together into 2 larger rotator assemblies (see Fig. 1(b).

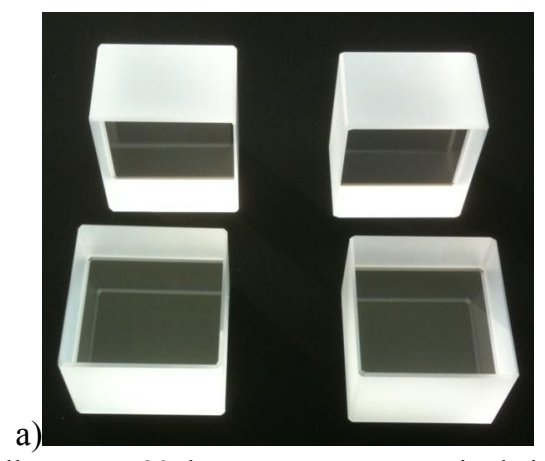

Fig. 1: (a) Four small aperture 90 degree quartz rotators in their rav state prior to edge polishing and edge bonding. (b) One of the bonded assemblies after polishing both of the broad faces. The angled bond line is only visible along the ground side face, which was not processed after bonding.

\section{BONDED QUARTZ ROTATOR EVALUATION}

The substrates were prepared by creating bonding surfaces at a 15 degree angle to the front surface normal. This geometry would assure that after bonding light transmitted through the rotator at normal incidence would not travel along the bond line which would be amorphous and induce phase error. At the 15 degree angle, the bond should be invisible to the transmitted phase of the beam assuming there is no induced stress in the substrate and there are no trapped contaminants along the bond interface. The results of this initial test are shown in Fig.2 where the "hairline" edge of the original parts is still visible. These edges were later removed in the final polishing step, but are left visible in the figure to illustrate the geometry and bring attention to the bond area because after polishing there should be no visible evidence that the bond even exists. To test the viability of this assembled quartz rotator, an experiment was performed with a schematic shown in Fig.3. A camera was used to assess the transmission of the parallel polarizers with the rotator cancelled by the waveplate (near 100\% transmission) and with the waveplate aligned to the polarization such that the 90 degree rotator creates a crossed polarization condition (near $0 \%$ transmission). The contrast between these two states was measured to be 1500:1 which is sufficient to determine the relative alignment of the two rotators within $\sim 1.5$ degrees. This assessment was made by translating the rotator orthogonal to the beam so that the beam could sample one half of the crystal, the bond area, and the other half successively. At each location the polarization contrast was evaluated. Initial measurements could find no difference in the transmitted intensity between the left and right halves 
including the bonded area. These results indicate excellent crystallographic alignment control between the two samples, exceeding the requirements of a LIFE rotator optic. The second test evaluated the reflectivity of the bond interface using a relative measurement method accomplished two ways: between the known Fresnel reflectivity from the surface and the reflected beam from the interface, or measure the extinguished intensity through crossed polarizers relative to the reflected beam from the interface. In both cases, this reflectivity was found to be $\sim 5 \times 10^{-6}$ indicating a very clean interface, very thin bond, and $<1.5$ degrees alignment of the crystalline axis.
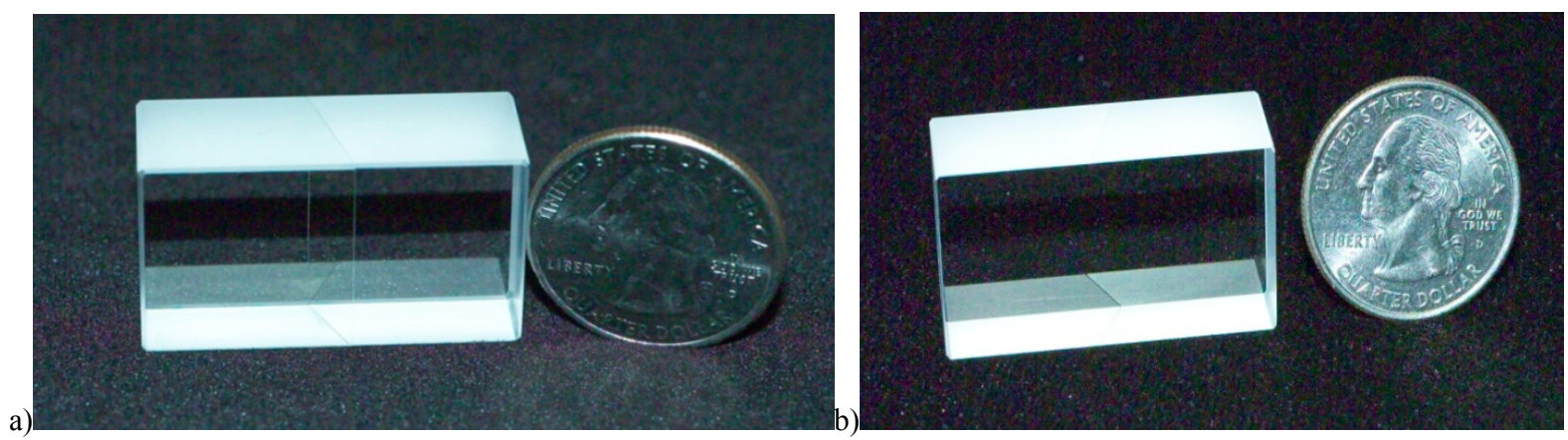

Fig. 2: a) small aperture 90 degree quartz rotators bonded together to create a single large aperture rotator. This unfinished substrate shows the residual edges of the original subcomponents which was removed by polishing in the finished part (b) to create a single contiguous aperture.

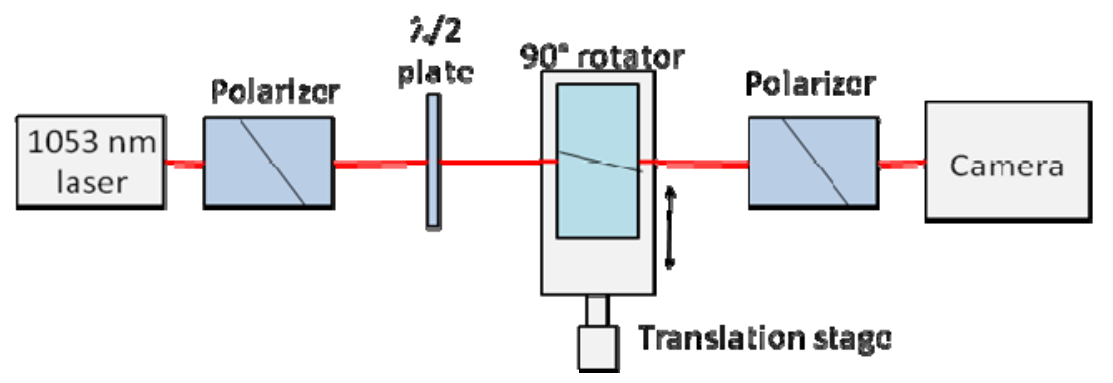

Fig. 3: Experimental setup for evaluating efficacy of bonded 90 degree quartz rotator

The final process step was to touch-up polish the input/output faces of the rotator assembly. This step was necessary to remove the fine seams of delamination at the outer edges of the bonded interface so that the bond line would be completely invisible to an incident laser. In addition, the final polishing step was needed to eliminate any path length differences through the rotator as a function of position. Such path length differences can arise whenever there is a slight ( $\sim 30$ arc second) misalignment of the 2 quartz pieces during bonding or if there is a small residual pyramidal error during the wedged face polishing step-see Fig.4. We discovered that the post-bond processing of the quartz assemblies was fairly difficult relative to ordinary glass, e.g., fused silica, assemblies due to the large sensitivity of the quartz assembly to thermal shock.

During our process development, we used one quartz assembly as a test piece for the more aggressive fabrication steps like coarse grinding. As a result, the outer surface of this first quartz assembly was damaged and rendered unsuitable for laser-based evaluation of the bonded interface. However, we were able to extract some useful information from the test piece by prying apart the bond. After exerting considerable force on the assembly in a direction parallel to the bond plane, we succeeded in separating the two quartz pieces in the assembly. Due to the very high bond strength, we saw that a major portion of the bonded interface did not delaminate-instead the bulk quartz material fractured above and below the original bond line (see Fig. 5). This bulk material fracture actually extended almost 400 microns above the plane of the original bond, which implies that there was negligible subsurface damage below the polished surfaces. It appears that the polished quartz material at the two mating surfaces was bonded so strongly that the bulk crystalline quartz fractured before the bond gave way. The observed high bond strength bodes well for future work that we have planned for larger edge-bonded quartz assemblies. 


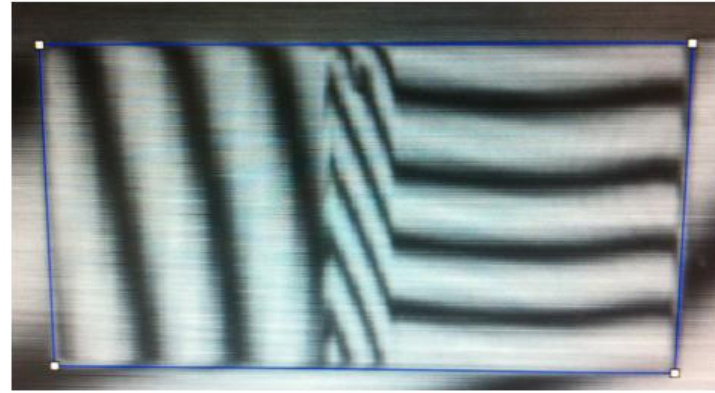

a)

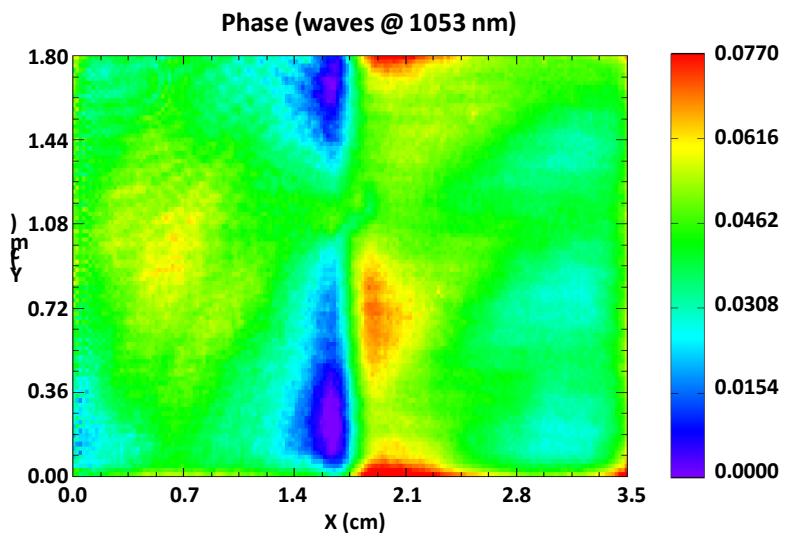

b)

Fig. 4: (a) Transmitted wave interferogram showing three discrete zones in the quartz assembly after initial bonding. Each zone has a different optical path length due to slight misalignment of the polished faces. (b) Transmitted wavefront error (TWE) OPD map after polishing of the input/output faces of the rotator assembly.

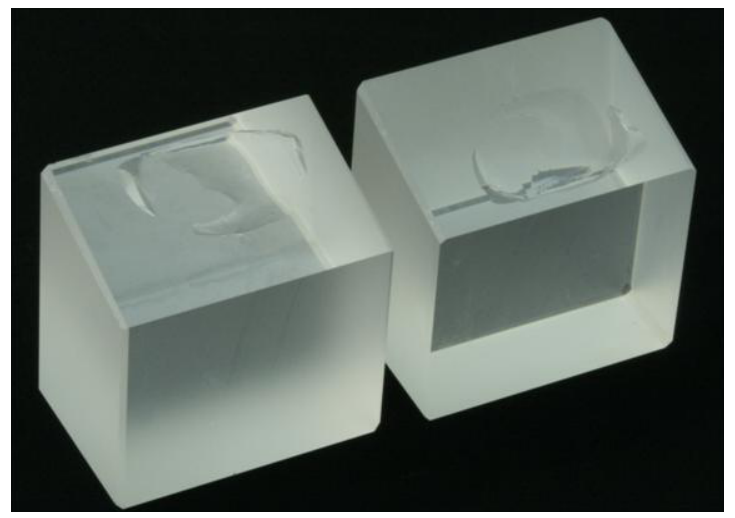

Fig. 5: Quartz rotator pieces after prying apart one of the bonded assemblies. Bulk material fracture zones are visible as elliptical regions near the center of each bonding surface. The shape of each zone is a mirror image of the other.

\section{CONCLUSIONS}

All of the above results are very promising for scaling this bonding technique to full aperture LIFE optics. The final polished part will now undergo further testing such as: transmitted wavefront and bond interface damage testing. In the interest of moving this technique forward quickly, larger substrates (each $5 \times 8 \mathrm{~cm}^{2}$ ) have already been procured and are being prepared for a larger aperture demonstration of this bonding technique.

\section{ACKNOWLEDGEMENTS}

This work was performed under the auspices of the U.S. Department of Energy by Lawrence Livermore National Laboratory under Contract DE-AC52-07NA27344 in conjunction with Precision Photonics Corp internal funding. 


\section{REFERENCES}

[1] C.A. Haynam et. al., "National Ignition Facility Laser Performance Status," Appl. Opt. 46, 3276 (2007).

[2] E.I. Moses, "Ignition on the National Ignition Facility: a path towards inertial fusion energy," Nuc. Fus., 49, 104022 (2009).

[3] M. Dunne et. al., "Timely Delivery Of Laser Inertial Fusion Energy (LIFE)," Fus. Sci. Tech., to be published 2011.

[4] J.F. Latkowski et. al., "Chamber Design for LIFE," Fus. Sci. Tech., to be published 2011.

[5] T. Anklam et. al., "LIFE System Design," Fus. Sci. Tech., to be published 2011

[6] Bayramian, et. al., "Compact, efficient laser systems required for laser inertial fusion energy," Fus. Sci. Tech., to be published 2011.

[7] N. Traggis and N. Claussen, "Improved bond strength characterization of chemically activated direct bonding (CADB) epoxy-free bonded solid state laser materials", SPIE Proceedings, Vol. 7578: Solid State Lasers XIX: Technology and Devices, (2010). 


\title{
Quartz rotators for Inertial Fusion Energy Lasers
}

\author{
A.J. Bayramian, K. I. Schaffers, J. Adams, J. Wolfe, C. Stolz, and R. J. Deri
}

One of the many optics required for the LIFE laser architecture is a large aperture $\left(\sim 27 \times 27 \mathrm{~cm}^{2}\right) 90 \mathrm{degree}$ quartz rotator for thermal birefringence compensation of the $\mathrm{Nd}$ :glass laser amplifiers. Such an optic is larger than any current quartz crystal and represents a new material from the standpoint of NIF qualified optical materials. Many years of growth scaling will be required to eventually scale the optic up to full aperture. A potential alternative to growing very large crystals is to optically bond smaller apertures together.

The Chemically Activated Direct Bonding process $\left(\mathrm{CADB}^{\circledR}\right)$ has been developed by Precision Photonics Corp and results in epoxy-free optical paths that are $100 \%$ optically transparent with negligible scattering and absorptive losses at the interfaces. For YAG and similar materials, the process has been proven to offer bond strength performance equivalent to that of bulk. It is thus exceptionally durable, reliable and resistant to changes in laser fluence, temperature, and humidity. ${ }^{1}$ Due to the zero bond line thickness, complete conformance is ensured between the two bonded surfaces, making it ideal for this application. Using the CADB process, a small aperture quartz rotator samples were bonded together to test this alternative.

Since the publication in 2010, the bonding has been scaled to achieve bonding of two $5.0 \times 8.0 \times 1.4 \mathrm{~cm} 3$ parts to achieve a final aperture of $8.0 \times 9.0 \times 1.4 \mathrm{~cm} 3$ bonded subsrate (fig. 1 a) with a finished wavefront (Fig. 1b). The transmitted wavefront (<lambda/14) \& rotation (>99.9\%) are reasonable for this first demo. The wavefront will improve with process knowledge and experience in bonding large parts, and can be corrected after bonding using deterministic finishing techniques such as MRF technology previously demonstrated.
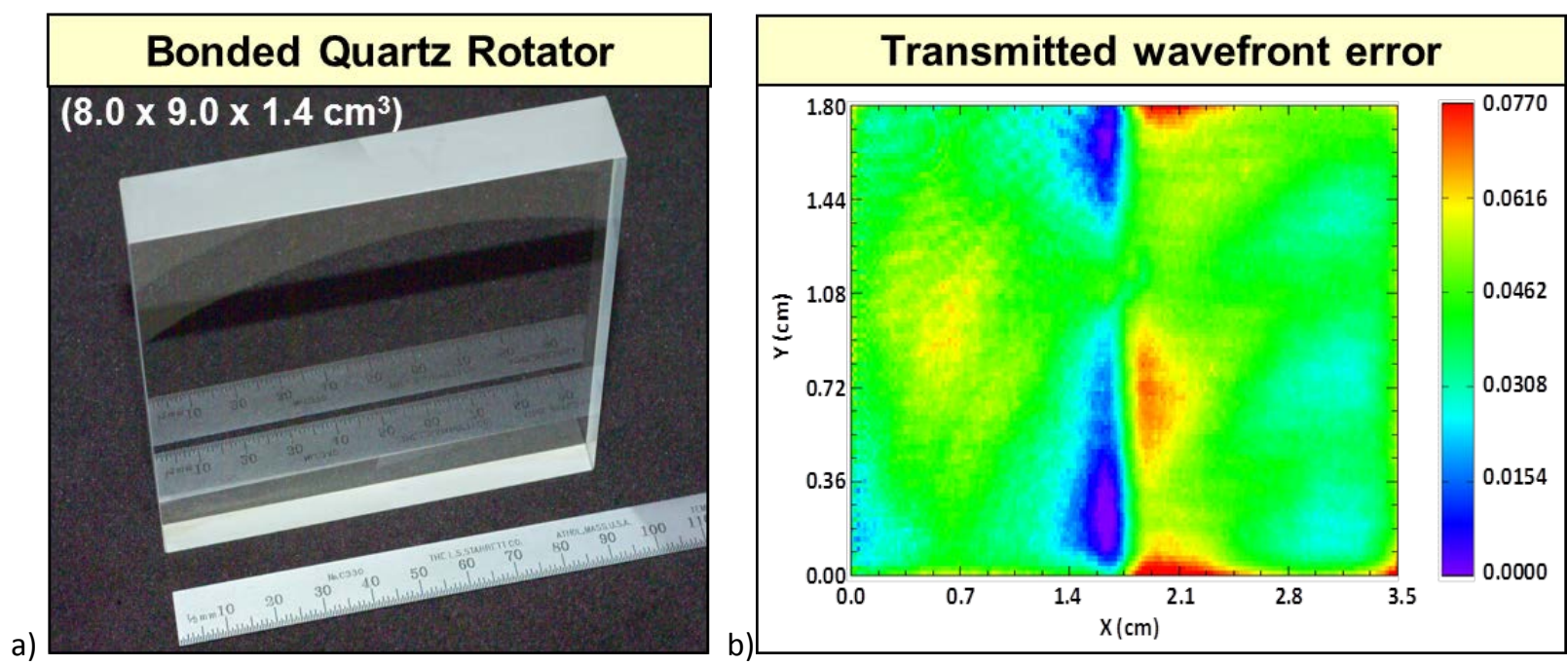

Fig. 1a) bonded quartz rotator, b) transmitted wavefront of bonded quartz rotator using same methodology as previously published by Traggis, et. al.

Using this bonding technique, full size LIFE aperture quartz rotators could be fabricated today by bonding four $15 \mathrm{x}$ $15 \mathrm{~cm} 2$ single crystal substrates available from Sawyer Corporation.

Another important aspect of these rotators is demonstration performance at high fluence (i.e. laser damage resistance). To get an understanding of where the material capabilities currently stand, we commissioned tests of a series of rotators from several vendors worldwide including Sawyer Corporation, a bonded sample from Carl Lambrecht, and Asahi Glass Company. Raster scan damage testing was carried out at SPICA Inc. on the three different samples over $1 \mathrm{~cm} 2$ area using a $1064 \mathrm{~nm}, 3 \mathrm{~ns} \mathrm{Nd:YAG} \mathrm{laser} \mathrm{system.} \mathrm{First} \mathrm{initiation} \mathrm{sights} \mathrm{were} \mathrm{observed}$ at $16 \mathrm{~J} / \mathrm{cm}^{2}, 16 \mathrm{~J} / \mathrm{cm}^{2}$ and $49 \mathrm{~J} / \mathrm{cm}^{2}$ respectively. The $49 \mathrm{~J} / \mathrm{cm} 2$ result was observed on the Sawyer part, but at this early evaluation stage this could very well be sample to sample variation. Rather the lesson we take from this is 
that the crystal can intrinsically have very high damage thresholds over $\mathrm{cm} 2$ apertures. This is a very good place to start engineering this material for larger aperture high damage threshold capability similar to the other optical materials development on the NIF. In addition to the raster scans, a long term test was performed at a single site at the nominal LIFE operational fluence to get an idea of intrinsic lifetime of this material. Using the same laser source, the site was successfully tested at $10 \mathrm{~J} / \mathrm{cm} 2$ for $10^{5}$ shots. This data nominal shows compliance with laser specifications and justifies further pursuit of this material for the intended application for high average power laser systems for IFE and other industrial and scientific uses.

\section{References}

1. N. Traggis, N. Claussen, A. Bayramian, K. Schaffers, and B. Deri, "Improved Characterization of Transmitted Wavefront Error on CADB ${ }^{\circledR}$ Epoxy-Free Bonded Solid State Laser Materials," SPIE, 7912, 79121k1-5, 2010. 


\section{Spatial filtering for IFE Lasers \\ A.J. Bayramian, A. C. Erlandson, S. Rana, K. I. Schaffers, M. Monticelli, M. A. Henesian, A. L. Bullington, G. Huete, and R. J. Deri}

A common technique used to improve beam quality in high energy systems is the use of a small aperture (pinhole) in a relay telescope as a spatial filter. This limits the gain of high spatial frequency near field amplitude modulation due to accumulation of nonlinear phase distortion, and holds off beamline parasitics. At high average power the grazing incidence cone pinholes typically used for this purpose exhibit plasma blow-off on each shot, since the fluence levels are significantly above the damage threshold of the material (typically steel, tungsten, or diamond pinhole). ${ }^{1,2}$ To alleviate this problem, IFE lasers will use a pair of longitudinally displaced, orthogonal cylindrical relay telescopes (Fig. 1).

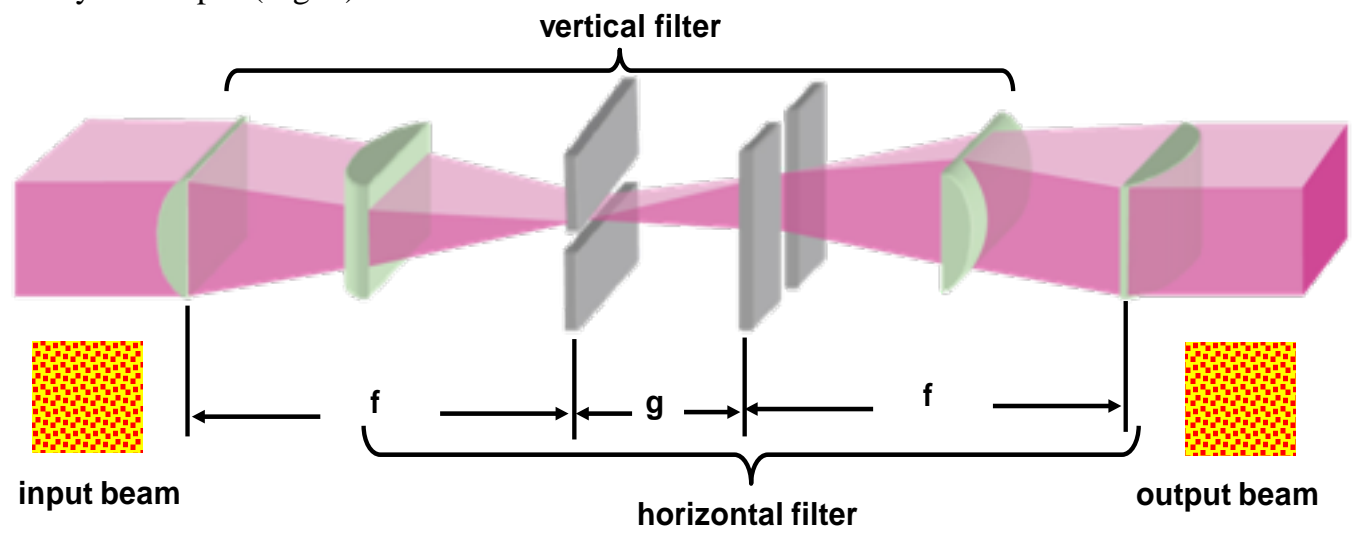

Fig. 1 Cylindrical spatial filter concept showing laser line focus thereby lowering the laser intensity on the filters enabling long lifetime

The longitudinal displacement of the $1 \mathrm{X}$ relay telescopes does not affect the object or relay image points in either axis, but does separate the foci in each axis thereby creating line-foci where this intensity is lower by $\sim 100 \mathrm{X}$. Filtering can then be accomplished with slits instead of pinholes at fluences engineered to be below the damage threshold of the slit material. Since the slits are at the far field in one axis, the net spatial filtering response vs. far field angle is the same as a square cone pinhole (which is ideally matched to the large square beams used on NIF, Mercury, and IFE). Note that while fig. 4 shows a single on-axis pinhole for clarity, a multiplexed version of this telescope can be realized by replacing each on-axis slit with two off-axis slits. Ablation and damage of the slit material can be avoided by employing a slit material such as fused silica at grazing incidence, which has a very high damage threshold $\left(\sim 475 \mathrm{GW} / \mathrm{cm}^{2}\right){ }^{3}$ This high threshold is due to the fact that most of the light is reflected at grazing incidence, and the light that is transmitted is only refracted and not absorbed by the fused silica. Our baseline design is to load the slit at only a fraction of this intensity, $\sim 3-30 \mathrm{GW} / \mathrm{cm}^{2}$. This is evidenced through diffractive beamline simulations based on benchmarked models used extensively by the NIF program (Fig.2 a-c). The LIFE laser system input nearfield (Fig. 2a) is focused by the first cylindrical lens to a line focus (Fig.2b). If we examine the this far field intensity profile (Fig. 3c), the advantage of cylindrical filtering becomes very clear. At the 200 microradian spatial filtering angle, the fluence is only $10-100 \mathrm{~J} / \mathrm{cm} 2$. At the infrared wavelength $(1053 \mathrm{~nm})$, uncoated fused silica typically demonstrates damage thresholds higher than this even at normal angle of incidence 

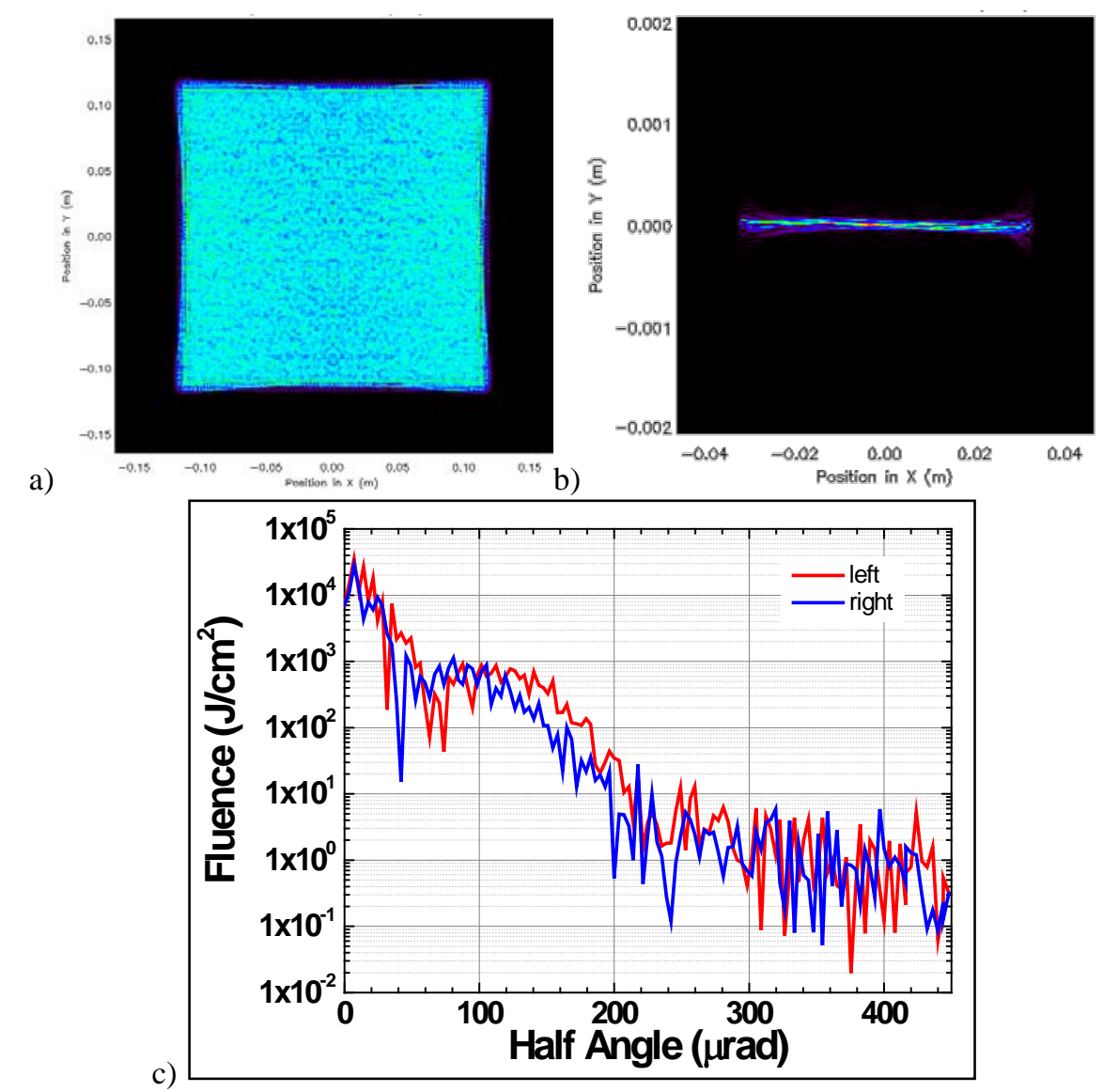

Fig. 2 a) Simulated nearfield profile of a beam entering the cavity spatial filter, b) far field profile (in one axis) of the beam, c) Line profile orthogonal to the far field shown in b) showing fluence vs. half angle.

To reduce this concept to practice, a complete $1 / 3$ scale telescope has been constructed for the purpose of field testing this concept (fig. 3). The purpose of this hardware is two-fold: to demonstrate a viable 1:1 cylindrical relay telescope with slit based spatial filtering, and to demonstrate the viability of these filters at high laser fluence.

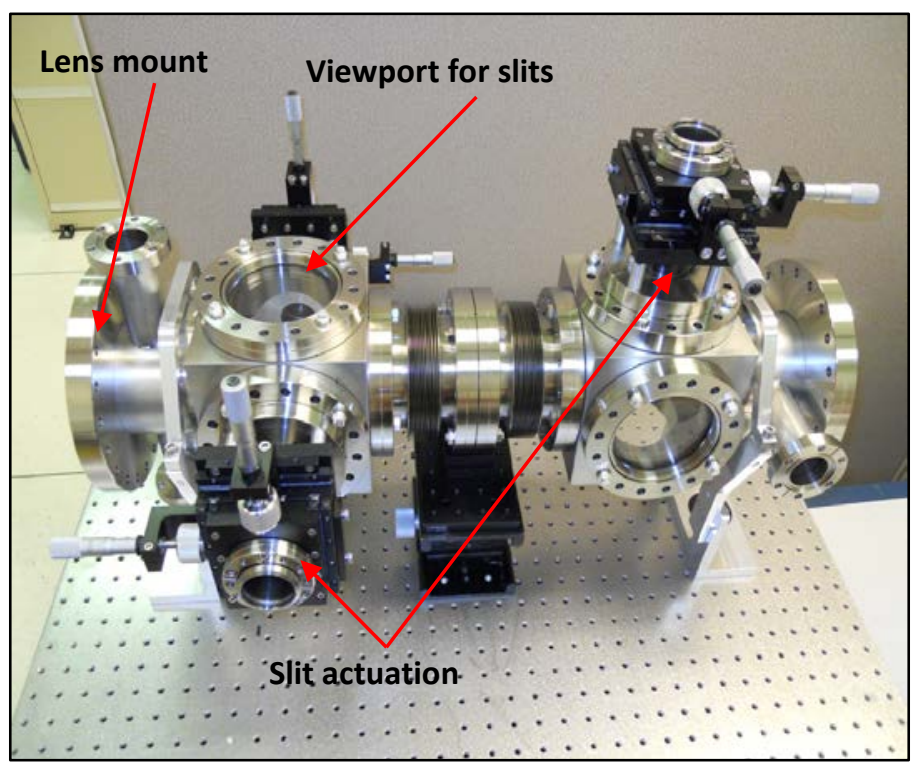

Fig. 3 Cylindrical spatial filter 
The first task is to independently demonstrate the viability of the silica based slit filters. A laser damage experiment (fig. 4a) was designed and implemented utilizing a prototype silica filter substrate at SPICA Inc., a company specializing in laser damage testing. Damage testing and handling protocols at SPICA have been validated and cross correlated with data taken at LLNL by NIF optics group. To simulate one half of a real slit spatial filter, a $50 \mathrm{x} 50 \mathrm{x}$ $10 \mathrm{~mm}^{3}$ fused silica substrate was irradiated at near grazing incidence. To maximize the performance of this filter substrate, we utilized fabrication, finishing, and handling techniques developed on NIF. The substrate is inclusion free Corning UV-grade 7980 fused silica which is polished on all sides. As a final finishing step, the surfaces were treated with the Acid Mitigation Process (AMP) ${ }^{4}$ developed by NIF scientists. This process cleans the surface at an atomic level, removing polishing contaminants and dulling scratch/dig flaws in the surface resulting in record level damage thresholds 2-3X beyond previous demonstration. While most of the light incident on the silica will be reflected via Fresnel reflection (Fig. 4b), some of the light will be transmitted into the substrate. The two large surfaces are wedged $2^{\circ}$ to prevent internal reflections from remaining trapped inside the substrate.

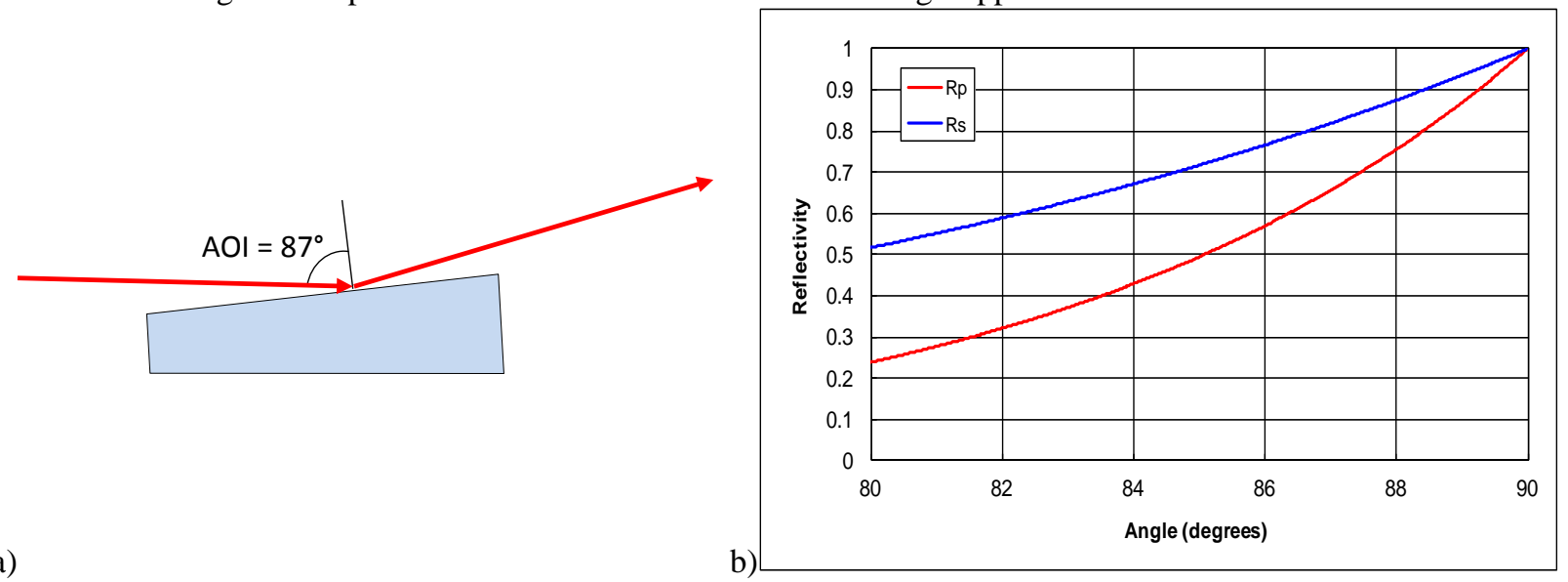

Fig 4 a) Silica filter experimental architecture, b) Fresnel reflectivity curves showing enhanced reflectivity near grazing incidence.

The testing laser is intended to mimic the conditions in a LIFE spatial filter. The test laser was a q-switched Nd:YAG laser at $1064 \mathrm{~nm}$ operating at $30 \mathrm{~Hz}$ with a $3 \mathrm{~ns}$ pulse and circular polarization. The laser beam was raster scanned at constant fluence over $80 \%$ of the full aperture at an 87 degree angle of incidence. The fluence was ramped from $10-300 \mathrm{~J} / \mathrm{cm}^{2}$ in the following increments: 10, 20, 30, 40, 50, 75, 100, 125, 150, 175, 200, 225, 250, 275, and $300 \mathrm{~J} / \mathrm{cm}^{2}$. Tests were performed first in dry air and then in vacuum to match the conditions on the inside of a vacuum relay telescope. The vacuum chamber with experimental apparatus is shown in Fig.5a, with a closeup of the laser test on the silica substrate in Fig. 5b. 

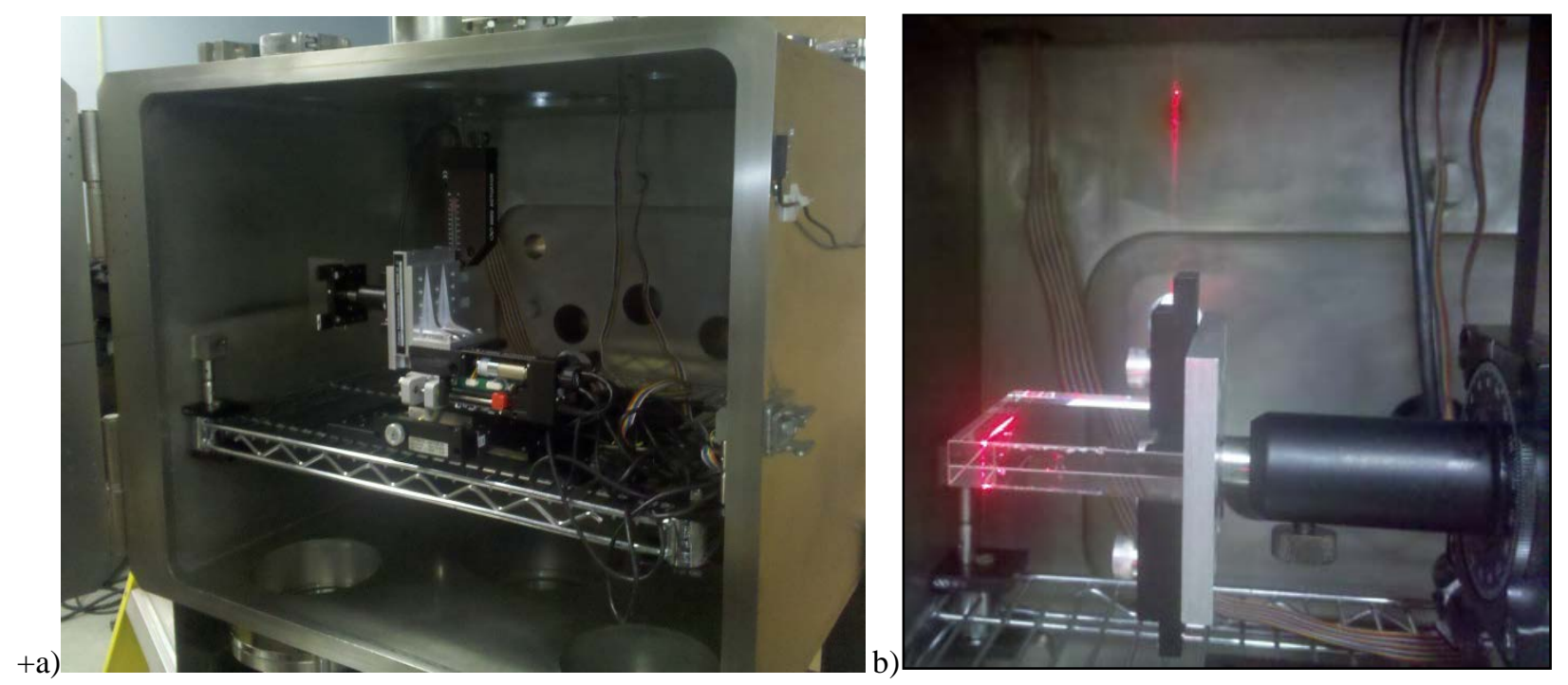

Fig. 5 a) Photo of the vacuum chamber used for the testing, b) closeup of the silica substrate showing the incident laser beam

For the experiment in air, a substrate was successfully ramped all the way to $300 \mathrm{~J} / \mathrm{cm} 2$ in dry air (RH 5\%). A second sample raster scanned in vacuum showed only a single damage spot initiated at $150 \mathrm{~J} / \mathrm{cm} 2$, which grew slightly at $275 \mathrm{~J} / \mathrm{cm} 2$, and the rest of the substrate successfully ramped to $300 \mathrm{~J} / \mathrm{cm} 2$. The sample tested in air was tested again at vacuum with no damage anywhere up to $300 \mathrm{~J} / \mathrm{cm} 2$. As a final "lifetime" test, the laser was held fixed in a single spot for 250,000 shots and showed no damage. These results confirm our expectations and the observations of Kurnit, and justify further investigation both in the form of these offline experiments and in integrated experiments using a full telescope and spatial filter assembly. We will be repeating these experiments in the future and add an RGA to the damage experiment to determine if any ablation of the surface is taking place. The absence of any measurable ablation of the surface would help to confirm the viability of these high average power spatial filters for long lifetime operation. When a high average power laser system is available, the integrated cylindrical spatial filter assembly will be tested as an integrated system to confirm these offline tests.

\section{References}

1. P.M. Celliers et al., "Spatial filter pinhole for high-energy pulsed lasers,” Appl. Opt., 37, 2371-2378 (1998).

2. J.E. Murray, D. Milam, C.D. Boley, K.G. Estabrook, and J.A. Caird, "Spatial filter pinhole development for the National Ignition Facility,“ Appl. Opt., 39, 1405-1420 (2000).

3. J. Kurnit et. al, SPIE, 3492, 896-900, 1999.

4. T.I. Suratwala, P.E. Miller, J.D. Bude, et al., "HF-Based Etching Processes for Improving Laser Damage Resistance of Fused Silica Optical Surfaces,” J. Am. Ceram. Soc., 94, 416-428, 2011. 